\title{
A New Route to 2'-O-Alkyl-2-thiouridine Derivatives via 4-O-Protection of the Uracil Base and Hybridization Properties of Oligonucleotides Incorporating These Modified Nucleoside Derivatives
}

\author{
Itaru Okamoto, ${ }^{1}$ Koh-ichiroh Shohda, ${ }^{1}$ Kohji Seio, ${ }^{2,3}$ and Mitsuo Sekine ${ }^{1,3, *}$ \\ ${ }^{1}$ Department of Life Science, Tokyo Institute of Technology \\ ${ }^{2}$ Frontier Collaborative Research Institute, Tokyo Institute of Technology \\ ${ }^{3}$ CREST, JST (Japan Science and Technology Corporation) \\ Nagatsuta, Midori-ku, Yokohama 226-8501, Japan
}

\section{Contents of Supporting Imformation}

General

${ }^{1} \mathrm{H}-\mathrm{NMR}$ spectrum of compound $\mathbf{1 a}$

${ }^{13} \mathrm{C}-\mathrm{NMR}$ spectrum of compound $\mathbf{1 a}$

${ }^{1} \mathrm{H}-\mathrm{NMR}$ spectrum of compound $\mathbf{1 b}$

${ }^{13} \mathrm{C}-\mathrm{NMR}$ spectrum of compound $\mathbf{1 b}$

${ }^{1} \mathrm{H}-\mathrm{NMR}$ spectrum of compound 1c

${ }^{13} \mathrm{C}-\mathrm{NMR}$ spectrum of compound $\mathbf{1 c}$

${ }^{1} \mathrm{H}-\mathrm{NMR}$ spectrum of compound $\mathbf{1 d}$

${ }^{13} \mathrm{C}-\mathrm{NMR}$ spectrum of compound $\mathbf{1 d}$

${ }^{1} \mathrm{H}-\mathrm{NMR}$ spectrum of compound $\mathbf{2 a}$

${ }^{13} \mathrm{C}-\mathrm{NMR}$ spectrum of compound $\mathbf{2 a}$

${ }^{1} \mathrm{H}-\mathrm{NMR}$ spectrum of compound $\mathbf{2 b}$

${ }^{13} \mathrm{C}-\mathrm{NMR}$ spectrum of compound $\mathbf{2} \mathbf{b}$

${ }^{1} \mathrm{H}-\mathrm{NMR}$ spectrum of compound $\mathbf{2 c}$

${ }^{13} \mathrm{C}-\mathrm{NMR}$ spectrum of compound $2 \mathrm{c}$

${ }^{1} \mathrm{H}-\mathrm{NMR}$ spectrum of compound $\mathbf{2 d}$

${ }^{13} \mathrm{C}-\mathrm{NMR}$ spectrum of compound $\mathbf{2 d}$

${ }^{1} \mathrm{H}-\mathrm{NMR}$ spectrum of compound $\mathbf{5}$

${ }^{13} \mathrm{C}-\mathrm{NMR}$ spectrum of compound $\mathbf{5}$

${ }^{1} \mathrm{H}-\mathrm{NMR}$ spectrum of compound $\mathbf{6}$ page $\mathrm{S} 4$

page $\mathrm{S} 5$

page $\mathrm{S} 6$

page $\mathrm{S} 7$

page $\mathrm{S} 8$

page $\mathrm{S} 9$

page $S 10$

page $S 11$

page $\mathrm{S} 12$

page $\mathrm{S} 13$

page $\mathrm{S} 14$

page $S 15$

page $S 16$

page $\mathrm{S} 17$

page $\mathrm{S} 18$

page $\mathrm{S} 19$

page $\mathbf{S} 20$

page $\mathrm{S} 21$

page $\mathrm{S} 22$

page $\mathrm{S} 23$ 
${ }^{13} \mathrm{C}-\mathrm{NMR}$ spectrum of compound $\mathbf{6}$

page S24

${ }^{1} \mathrm{H}-\mathrm{NMR}$ spectrum of compound 7

page $\mathrm{S} 25$

${ }^{13} \mathrm{C}$-NMR spectrum of compound 7

page $\mathrm{S} 26$

${ }^{1} \mathrm{H}-\mathrm{NMR}$ spectrum of compound $\mathbf{8}$

page $\mathrm{S} 27$

${ }^{13} \mathrm{C}-\mathrm{NMR}$ spectrum of compound $\mathbf{8}$

page $\mathrm{S} 28$

${ }^{1} \mathrm{H}-\mathrm{NMR}$ spectrum of compound $\mathbf{3 b}$

page $\mathrm{S} 29$

${ }^{13} \mathrm{C}-\mathrm{NMR}$ spectrum of compound $\mathbf{3 b}$

page $\mathrm{S} 30$

${ }^{1} \mathrm{H}-\mathrm{NMR}$ spectrum of compound 9

page $\mathrm{S} 31$

${ }^{13} \mathrm{C}-\mathrm{NMR}$ spectrum of compound 9

page $\mathrm{S} 32$

${ }^{1} \mathrm{H}-\mathrm{NMR}$ spectrum of compound $\mathbf{1 0}$

page $\mathrm{S} 33$

${ }^{13} \mathrm{C}-\mathrm{NMR}$ spectrum of compound $\mathbf{1 0}$

page $\mathrm{S} 34$

${ }^{1} \mathrm{H}-\mathrm{NMR}$ spectrum of compound $\mathbf{1 1}$

page $\mathrm{S} 35$

${ }^{13} \mathrm{C}-\mathrm{NMR}$ spectrum of compound $\mathbf{1 1}$

page S36

${ }^{1} \mathrm{H}-\mathrm{NMR}$ spectrum of compound $\mathbf{3 c}$

pageS37

${ }^{13} \mathrm{C}-\mathrm{NMR}$ spectrum of compound $\mathbf{3 c}$

page $\mathrm{S} 38$

${ }^{1} \mathrm{H}-\mathrm{NMR}$ spectrum of compound $\mathbf{1 2}$

page $S 39$

${ }^{13} \mathrm{C}-\mathrm{NMR}$ spectrum of compound $\mathbf{1 2}$

page $\mathrm{S} 40$

${ }^{1} \mathrm{H}-\mathrm{NMR}$ spectrum of compound $\mathbf{1 3}$

page $\mathrm{S} 41$

${ }^{13} \mathrm{C}-\mathrm{NMR}$ spectrum of compound $\mathbf{1 3}$

page $\mathrm{S} 42$

${ }^{1} \mathrm{H}-\mathrm{NMR}$ spectrum of compound $\mathbf{1 4}$

page $\mathrm{S} 43$

${ }^{13} \mathrm{C}-\mathrm{NMR}$ spectrum of compound $\mathbf{1 4}$

page $\mathrm{S} 44$

${ }^{1} \mathrm{H}-\mathrm{NMR}$ spectrum of compound $\mathbf{1 5}$

page $\mathrm{S} 45$

${ }^{13} \mathrm{C}-\mathrm{NMR}$ spectrum of compound $\mathbf{1 5}$

page $\mathrm{S} 46$

${ }^{1} \mathrm{H}-\mathrm{NMR}$ spectrum of compound $\mathbf{1 6}$

page $\mathrm{S} 47$

${ }^{13} \mathrm{C}$-NMR spectrum of compound $\mathbf{1 6}$

page $\mathrm{S} 48$

${ }^{1} \mathrm{H}-\mathrm{NMR}$ spectrum of compound $\mathbf{1 7}$

page $\mathrm{S} 49$

${ }^{13} \mathrm{C}-\mathrm{NMR}$ spectrum of compound $\mathbf{1 7}$

page $\mathrm{S} 50$

${ }^{1} \mathrm{H}-\mathrm{NMR}$ spectrum of compound $\mathbf{1 8}$

page $\mathrm{S} 51$

${ }^{13} \mathrm{C}-\mathrm{NMR}$ spectrum of compound $\mathbf{1 8}$

page $\mathrm{S} 52$

${ }^{1} \mathrm{H}-\mathrm{NMR}$ spectrum of compound $\mathbf{3 d}$

page $\mathrm{S} 53$

${ }^{13} \mathrm{C}-\mathrm{NMR}$ spectrum of compound $\mathbf{3 d}$

page $\mathrm{S} 54$

${ }^{1} \mathrm{H}-\mathrm{NMR}$ spectrum of compound $\mathbf{2 0}$

page S55

${ }^{13} \mathrm{C}-\mathrm{NMR}$ spectrum of compound $\mathbf{2 0}$

page $\mathrm{S} 56$

${ }^{1} \mathrm{H}-\mathrm{NMR}$ spectrum of compound 21

page $\mathrm{S} 57$

${ }^{13} \mathrm{C}-\mathrm{NMR}$ spectrum of compound $\mathbf{2 1}$

page $\mathrm{S} 58$ 
${ }^{1} \mathrm{H}-\mathrm{NMR}$ spectrum of compound 23

page S59

${ }^{13} \mathrm{C}-\mathrm{NMR}$ spectrum of compound $\mathbf{2 3}$

page $\mathrm{S} 60$

${ }^{1} \mathrm{H}-\mathrm{NMR}$ spectrum of compound $\mathbf{3 e}$

page $\mathrm{S} 61$

${ }^{13} \mathrm{C}-\mathrm{NMR}$ spectrum of compound $\mathbf{3 e}$

page $\mathrm{S} 62$

${ }^{1} \mathrm{H}-\mathrm{NMR}$ spectrum of compound $\mathbf{2 4 c}$

page $\mathrm{S} 63$

${ }^{13} \mathrm{C}$-NMR spectrum of compound $\mathbf{2 4 c}$

page $\mathrm{S} 64$

${ }^{1} \mathrm{H}-\mathrm{NMR}$ spectrum of compound $\mathbf{2 4 d}$

page $\mathrm{S} 65$

${ }^{13} \mathrm{C}-\mathrm{NMR}$ spectrum of compound $\mathbf{2 4 d}$

page $\mathrm{S} 66$

${ }^{1} \mathrm{H}-\mathrm{NMR}$ spectrum of compound $\mathbf{2 4} \mathbf{e}$

page $\mathrm{S} 67$

${ }^{13} \mathrm{C}$-NMR spectrum of compound $\mathbf{2 4} \mathbf{e}$

page $\mathrm{S} 68$

${ }^{1} \mathrm{H}-\mathrm{NMR}$ spectrum of compound $25 \mathrm{c}$

page $\mathrm{S} 69$

${ }^{13} \mathrm{C}-\mathrm{NMR}$ spectrum of compound $25 \mathrm{c}$

page $S 70$

${ }^{31} \mathrm{P}-\mathrm{NMR}$ spectrum of compound 25c

page $S 71$

${ }^{1} \mathrm{H}-\mathrm{NMR}$ spectrum of compound $\mathbf{2 5 d}$

page $\mathrm{S} 72$

${ }^{13} \mathrm{C}-\mathrm{NMR}$ spectrum of compound $\mathbf{2 5 d}$

page $\mathrm{S} 73$

${ }^{31} \mathrm{P}-\mathrm{NMR}$ spectrum of compound 25d

page $S 74$

${ }^{1} \mathrm{H}-\mathrm{NMR}$ spectrum of compound $25 \mathrm{e}$

page $\mathrm{S} 75$

${ }^{13} \mathrm{C}-\mathrm{NMR}$ spectrum of compound $\mathbf{2 5 e}$

page S76

${ }^{31} \mathrm{P}-\mathrm{NMR}$ spectrum of compound $\mathbf{2 5 e}$

page $\mathrm{S} 77$ 
General. ${ }^{1} \mathrm{H}$ NMR spectra were recorded at $270 \mathrm{MHz}$ and the chemical shifts were measured from tetramethylsilane (in $\mathrm{CDCl}_{3}$ ) as an internal standard or DSS (in $\left.\mathrm{D}_{2} \mathrm{O}\right)$ as an external standard. ${ }^{13} \mathrm{C}$ NMR spectra were recorded at $68 \mathrm{MHz}$ and the chemical shifts were measured from $\mathrm{CDCl}_{3}(77 \mathrm{ppm})$ as an internal standard or DSS $\left(0 \mathrm{ppm}\right.$, in $\left.\mathrm{D}_{2} \mathrm{O}\right)$ as the external standard. ${ }^{31} \mathrm{P}$ NMR spectra were recorded at $109 \mathrm{MHz}$ and the chemical shifts were measured from $85 \% \mathrm{H}_{3} \mathrm{PO}_{4}$ as an external standard. The ${ }^{1} \mathrm{H}-{ }^{1} \mathrm{H}$ coupling constants were measured at $400 \mathrm{MHz}$ at $25^{\circ} \mathrm{C}$. Pyridine was distilled twice from $p$-toluenesulfonyl chloride and $\mathrm{CaH}_{2}$ after being refluxed for several hours and stored over molecular sieves 4A. The other dry solvents were purchased and stored over molecular sieves 4A. TLC was performed on precoated glass plates of silica gel. Column chromatography was carried out with silica gel C-200 and C-60. The solid-phase synthesis of oligonucleotides was carried out on a DNA/RNA synthesizer model 392 using normal phosphoramidite protocol. Reversed-phase HPLC was performed using a C18 column with a linear gradient (0-30\%) starting from 0.1 $\mathrm{M} \mathrm{NH}_{4} \mathrm{OAc}(\mathrm{pH} 7.0)$ and applying $\mathrm{CH}_{3} \mathrm{CN}$ at a flow rate of $1.0 \mathrm{~mL} / \mathrm{min}$ for $30 \mathrm{~min}$ at room temperature. Anion-exchange HPLC was performed on an HPLC apparatus using a FAX column with a linear gradient (0-25\%) starting from 25 $\mathrm{mM}$ phosphate buffer ( $\mathrm{pH}$ 6.0) and applying $25 \mathrm{mM}$ phosphate buffer containing $1 \mathrm{M} \mathrm{NaCl}$ (pH 6.0) at a flow rate of $1.0 \mathrm{~mL} / \mathrm{min}$ at $50{ }^{\circ} \mathrm{C}$. The MALDI-TOF mass spectrometry was carried out. 


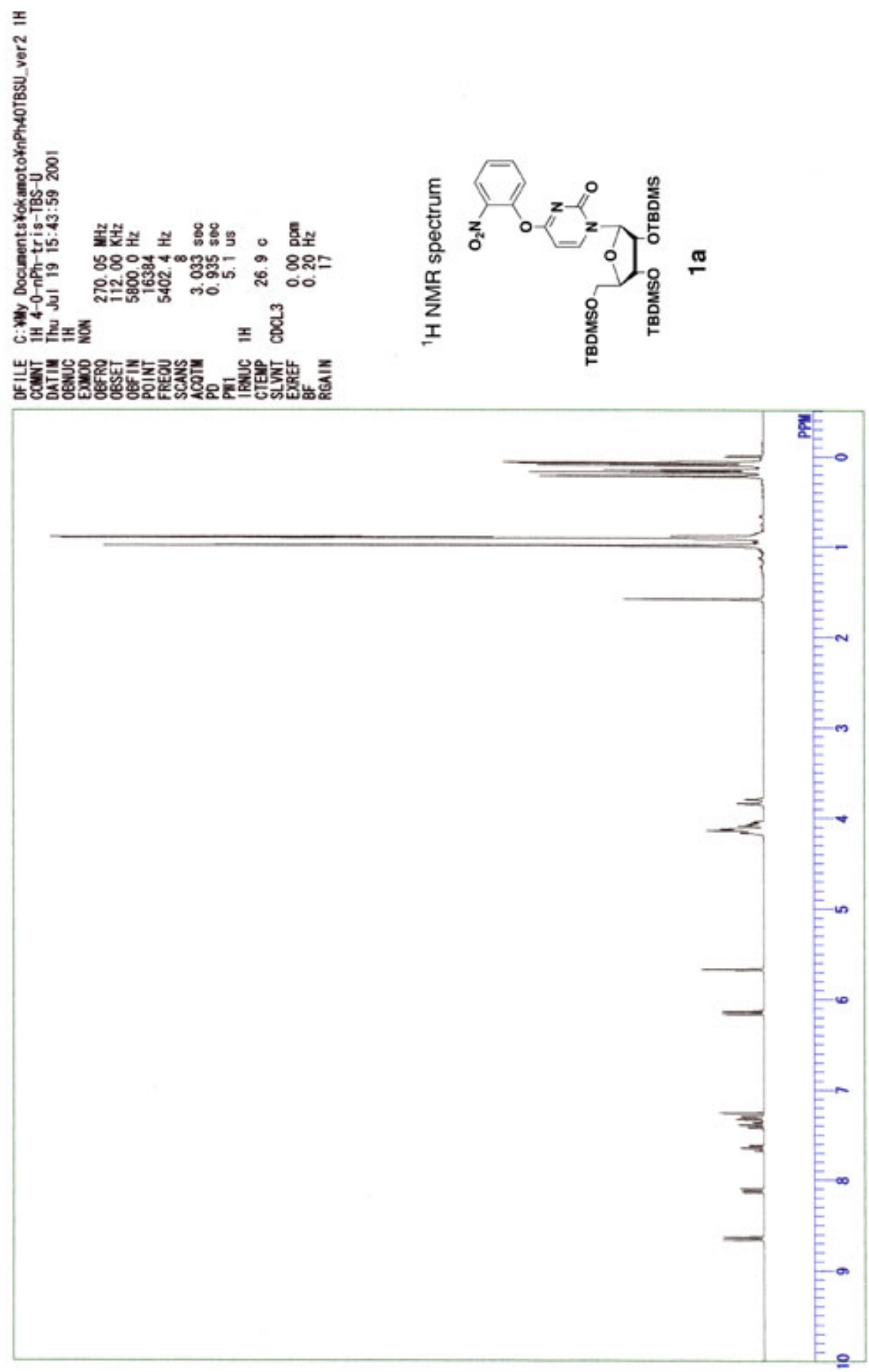



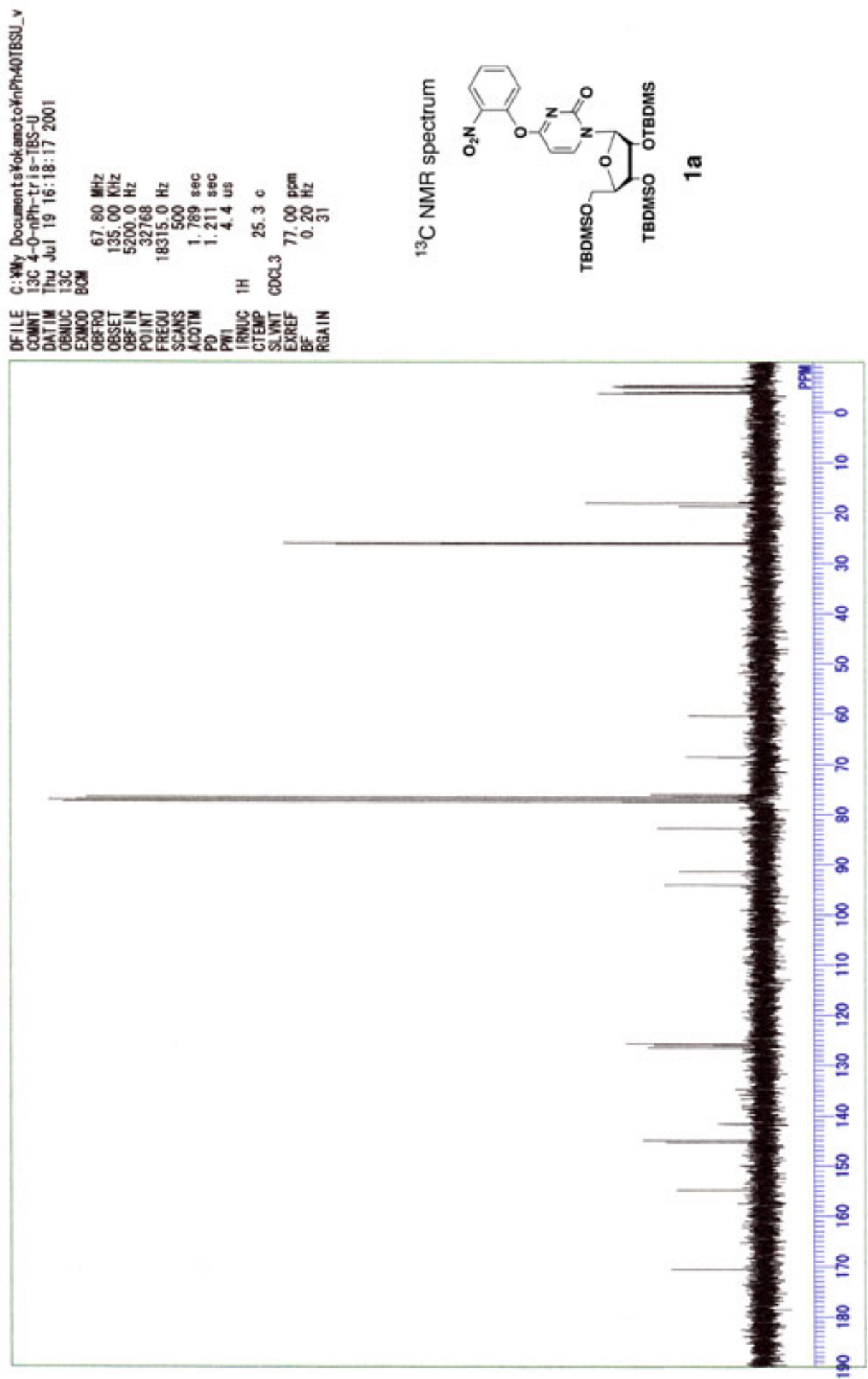


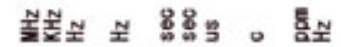

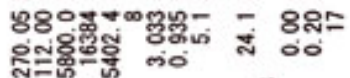

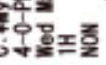

× 쯍

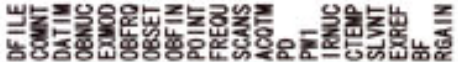

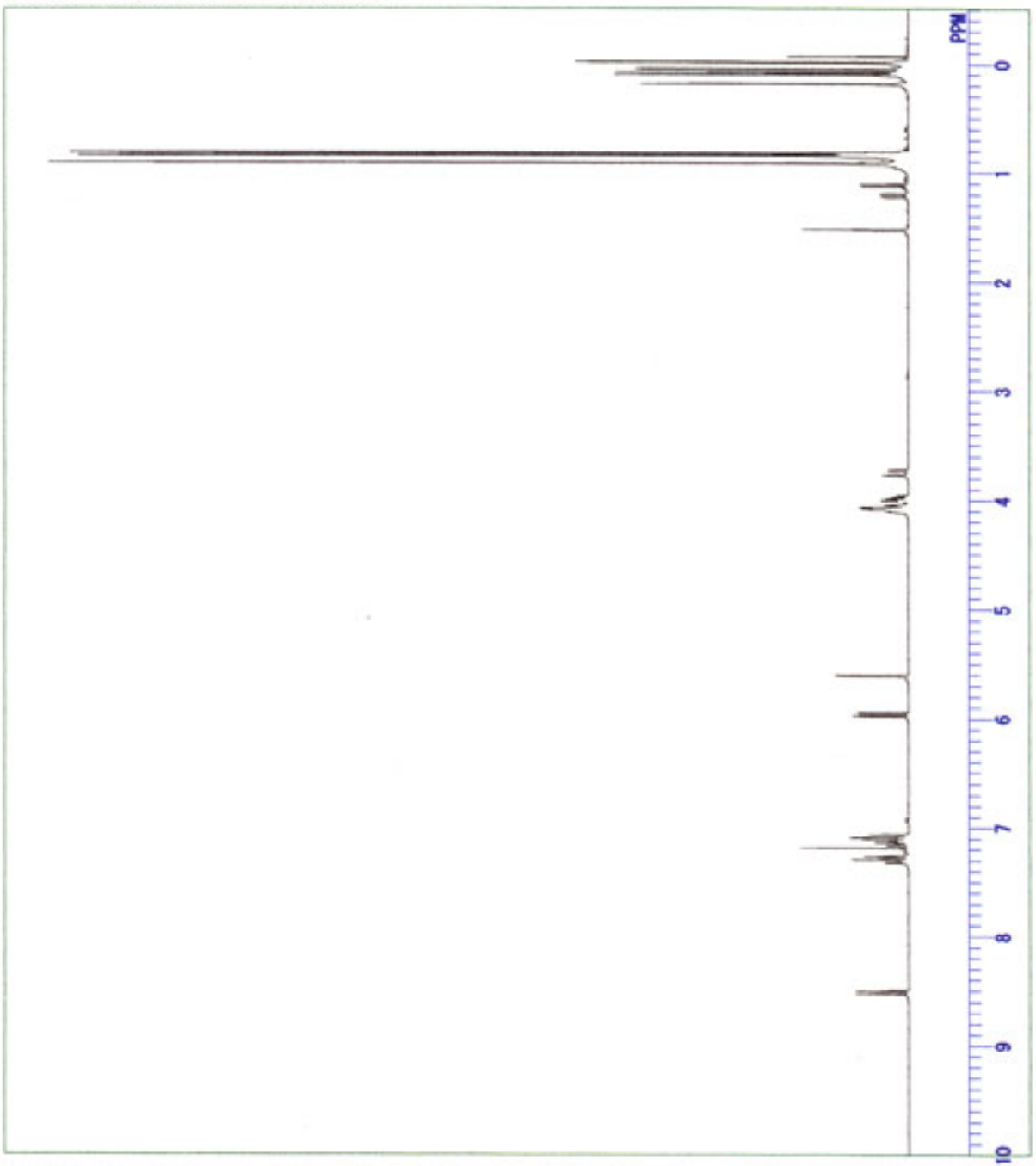



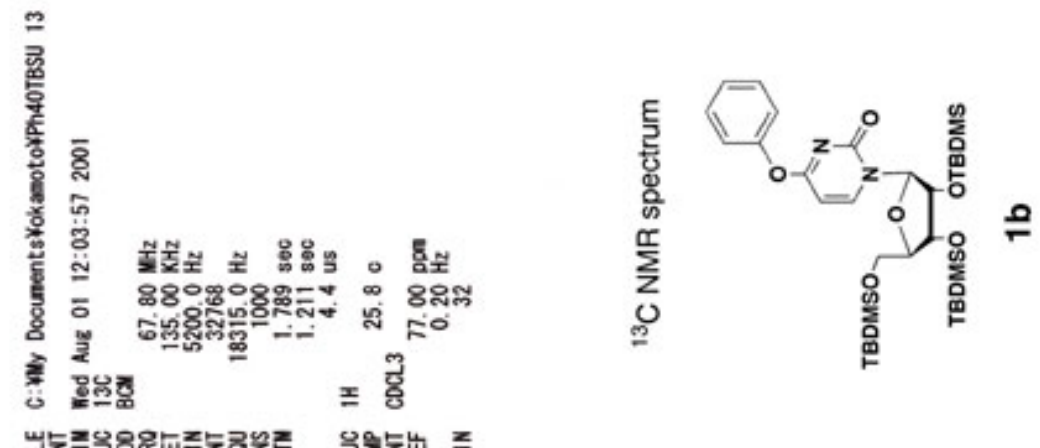

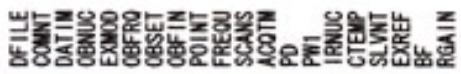

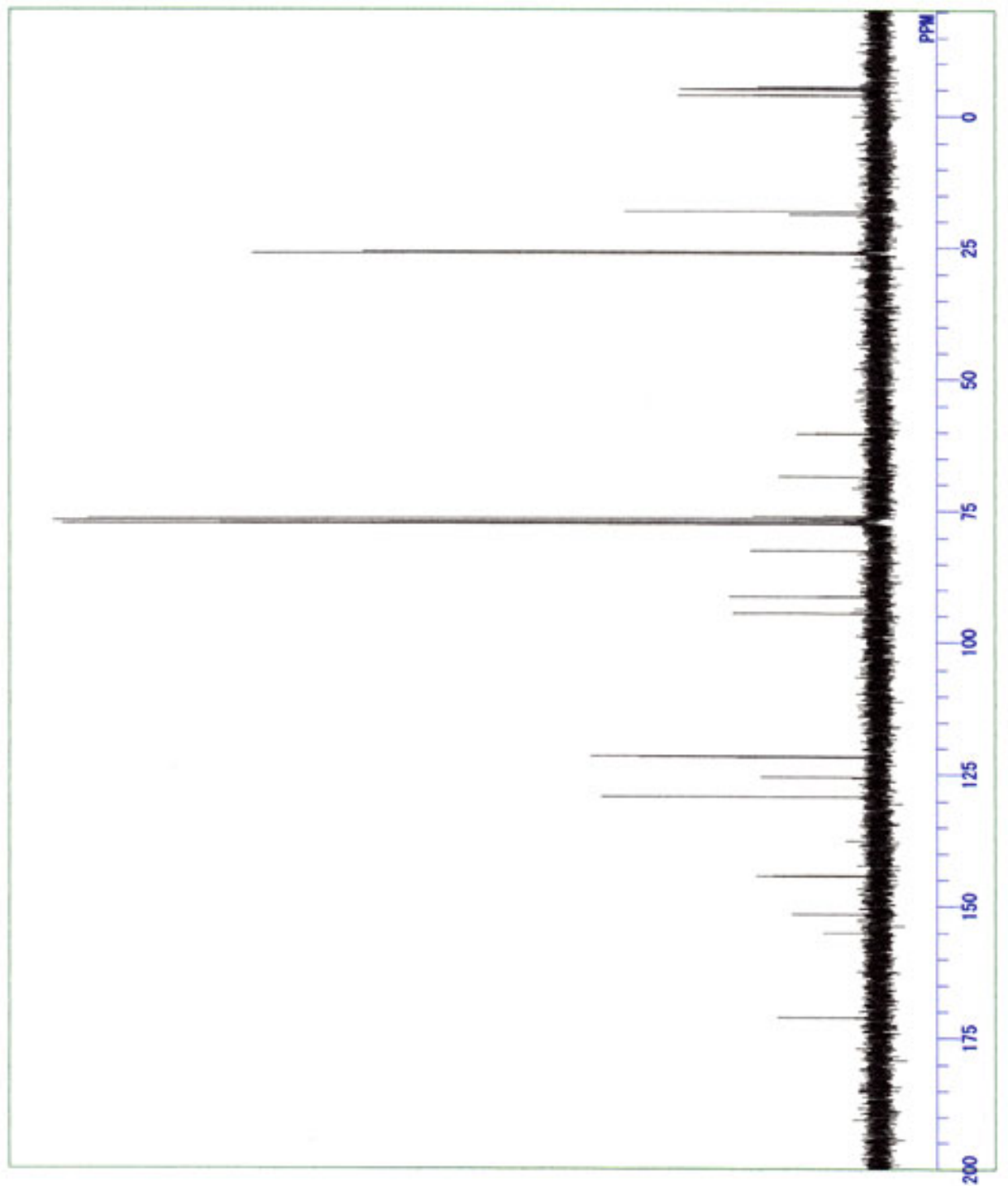



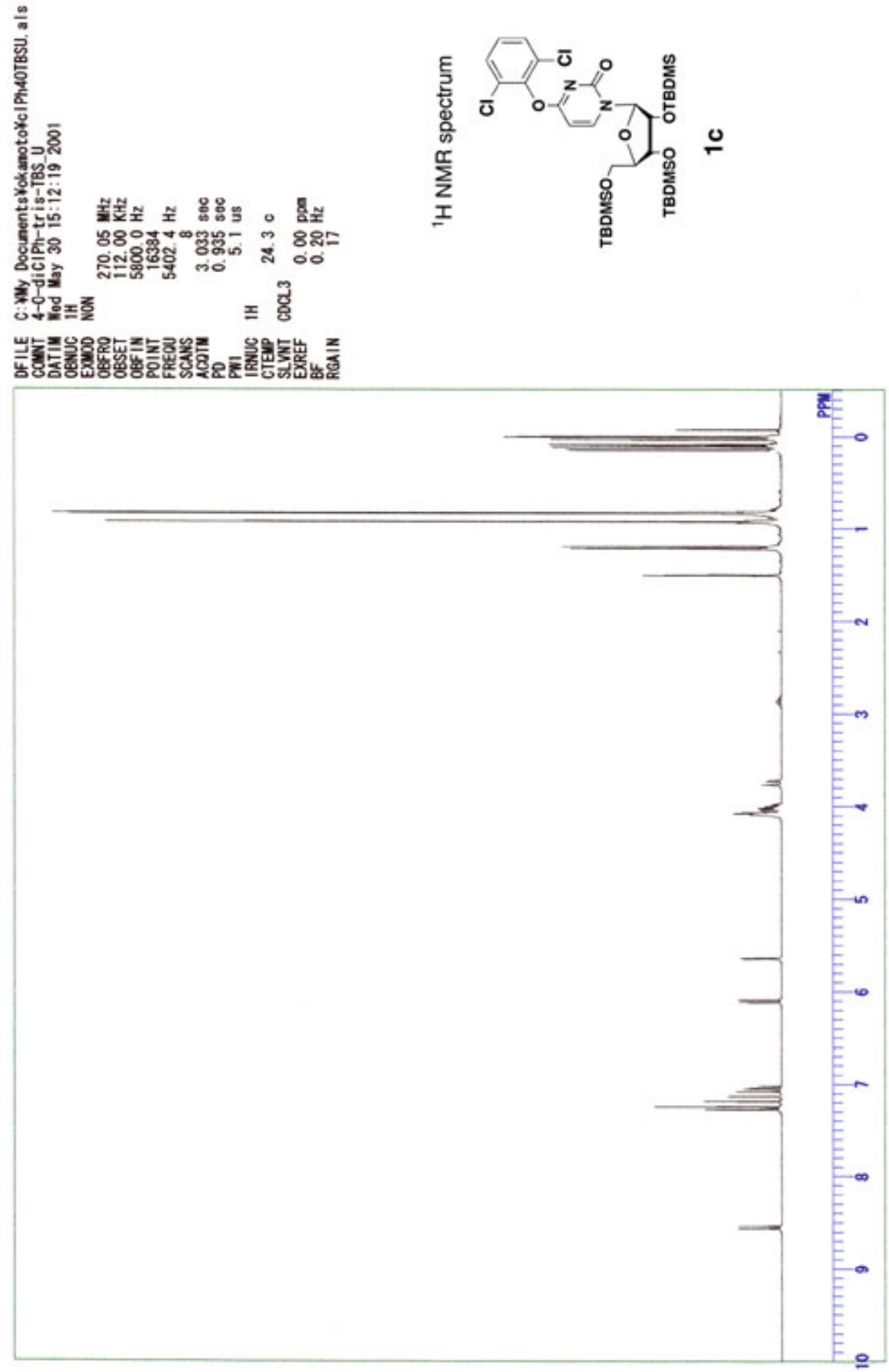

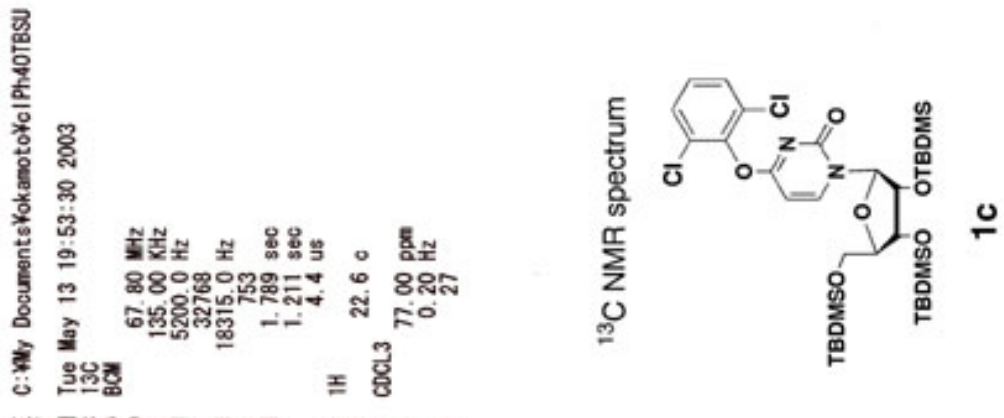

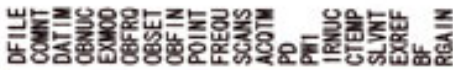

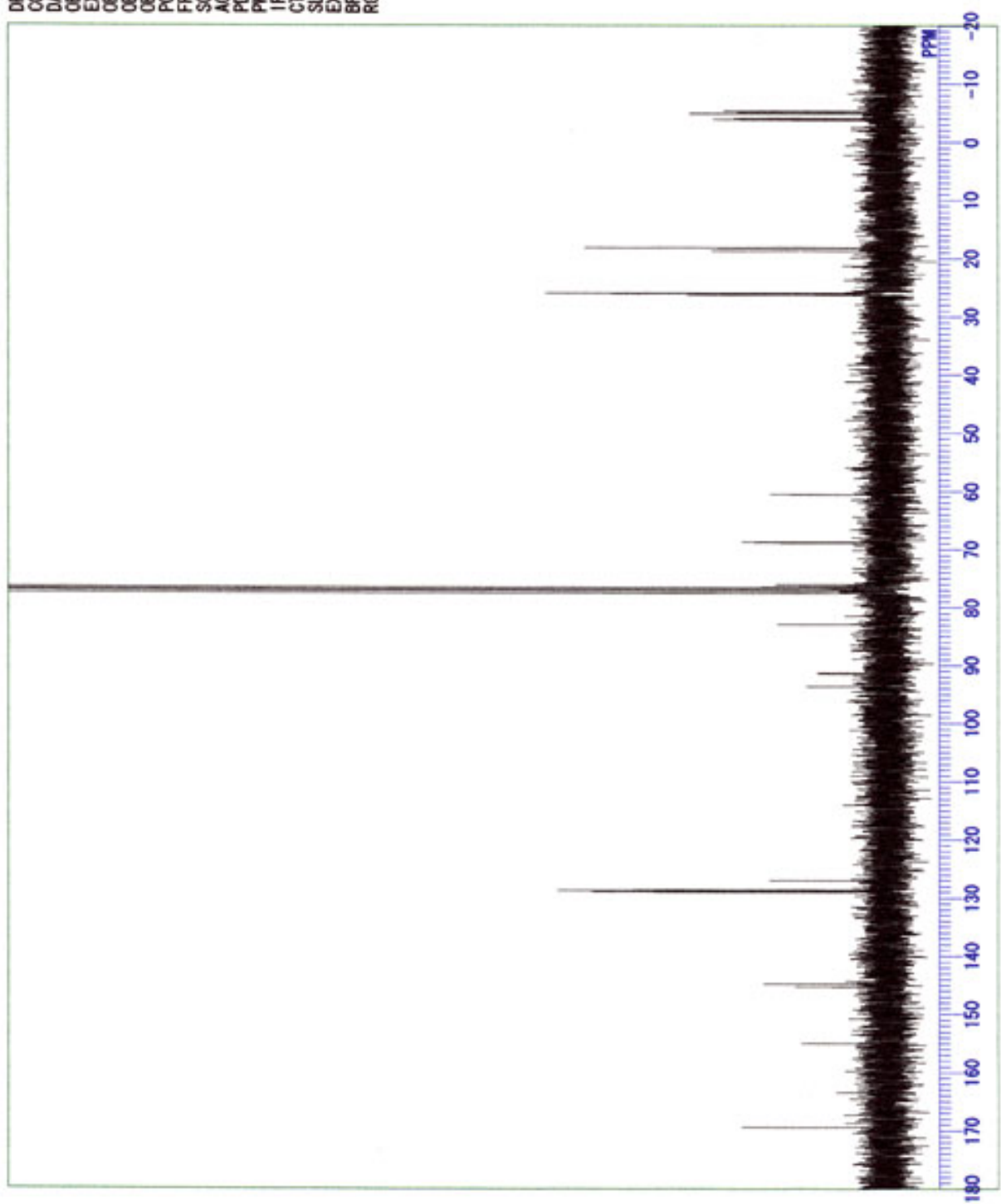



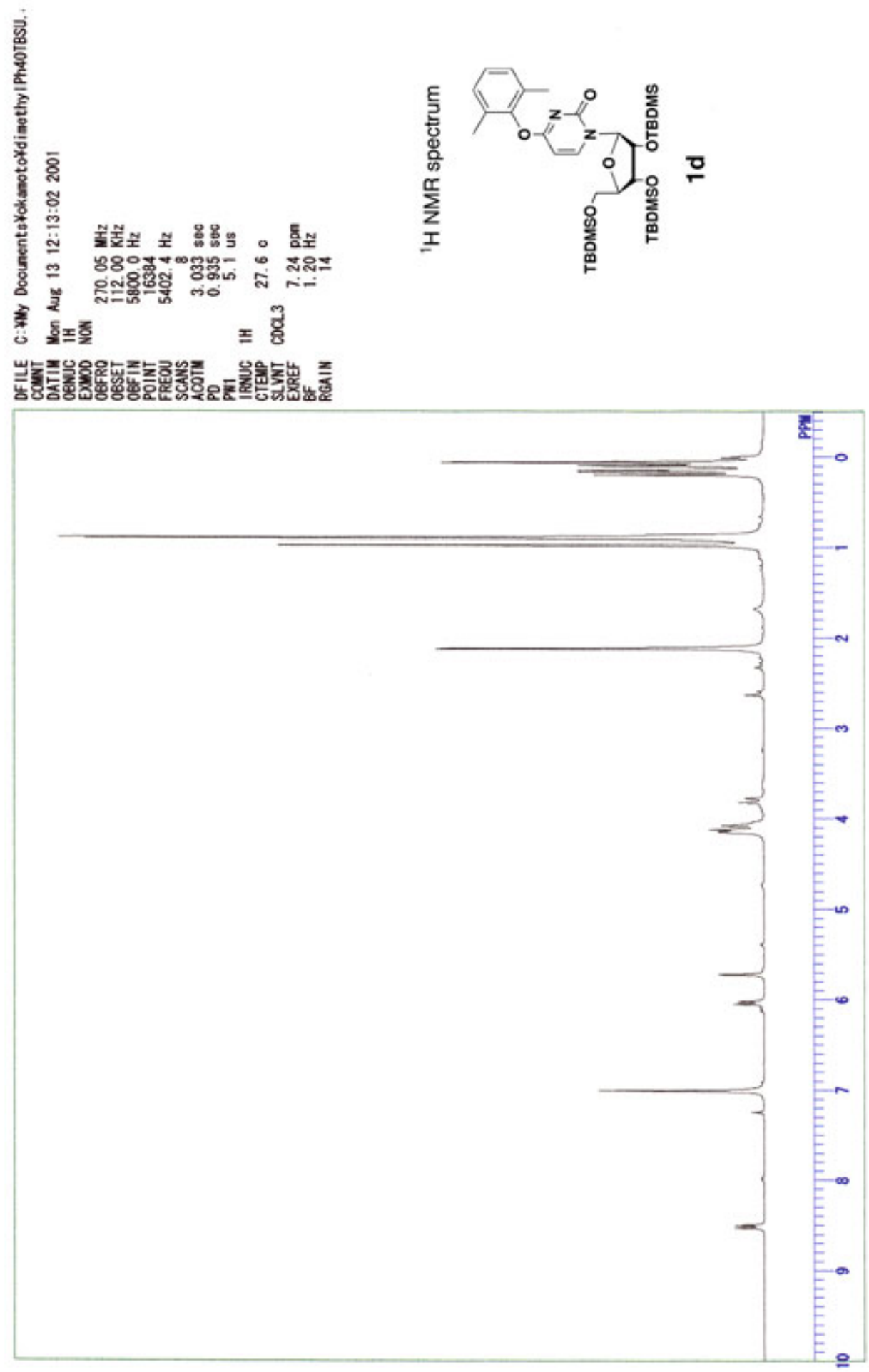

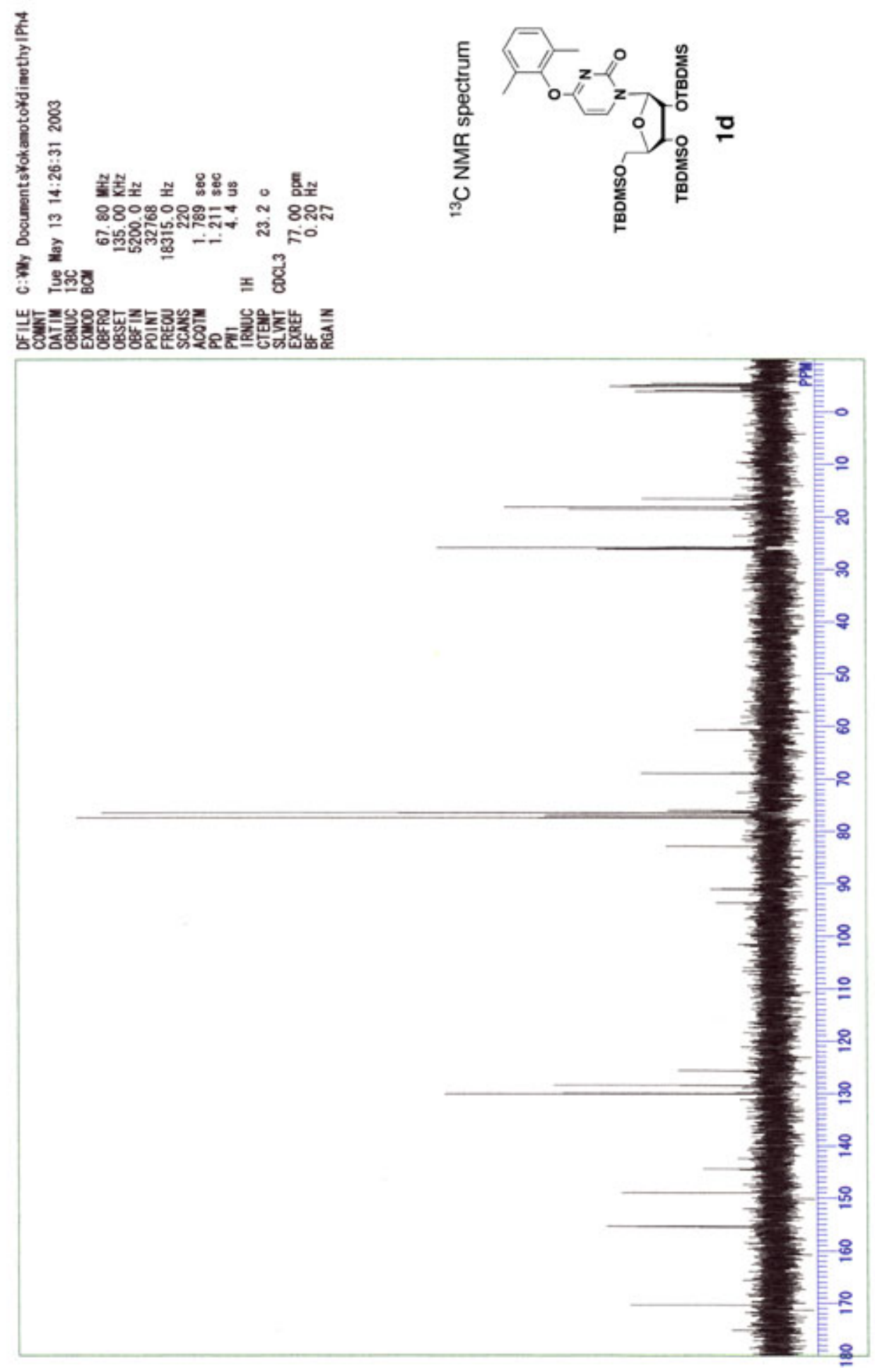


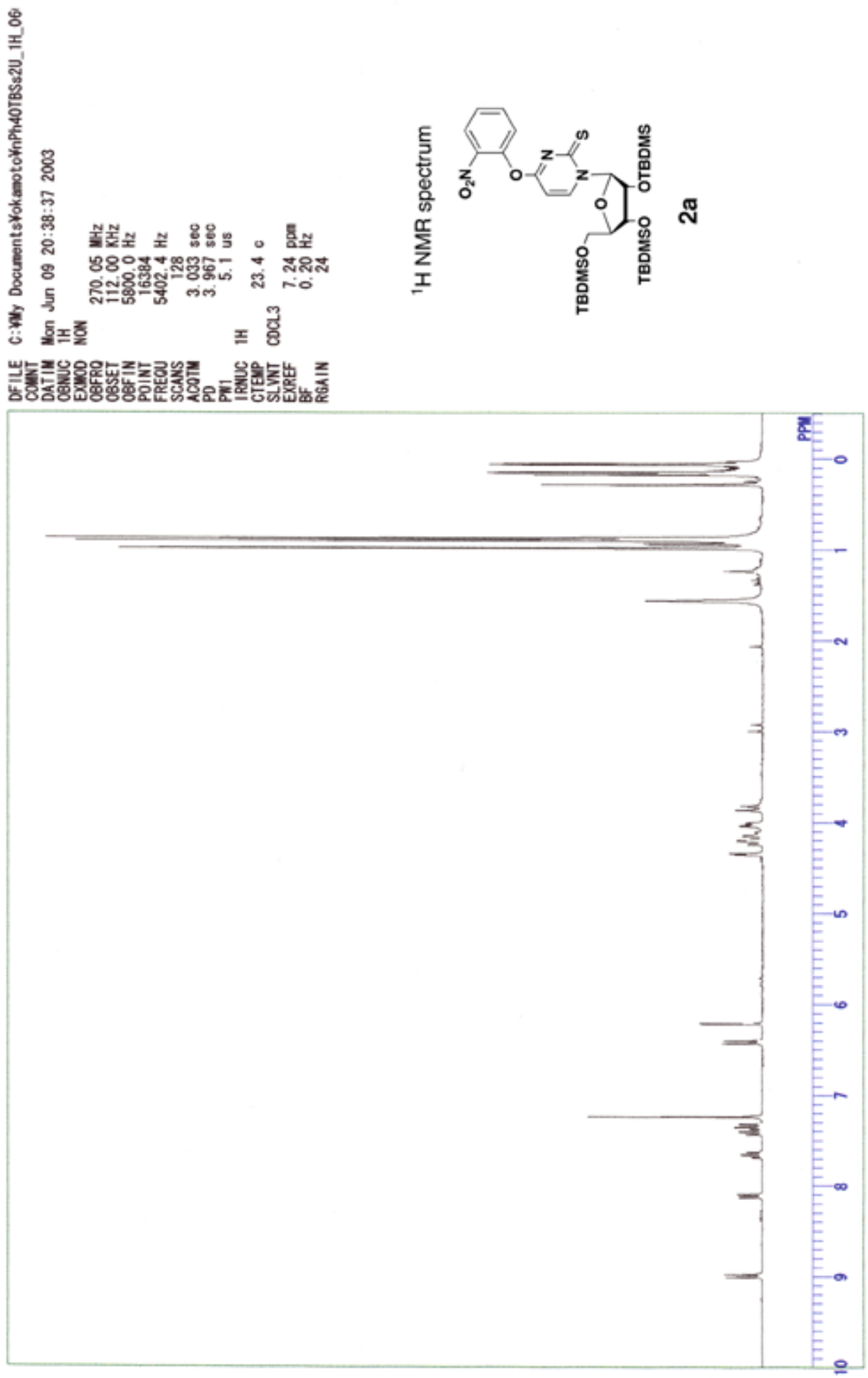



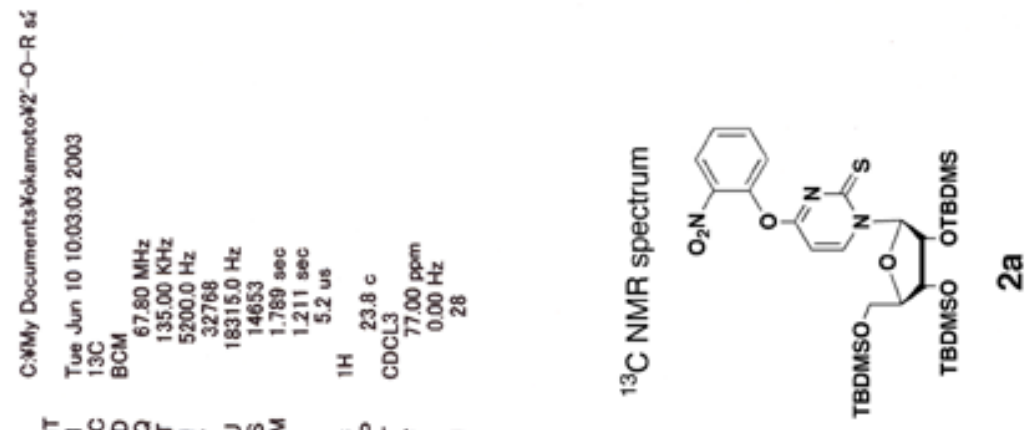

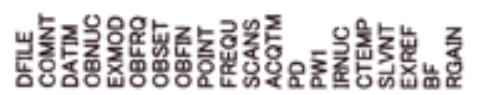

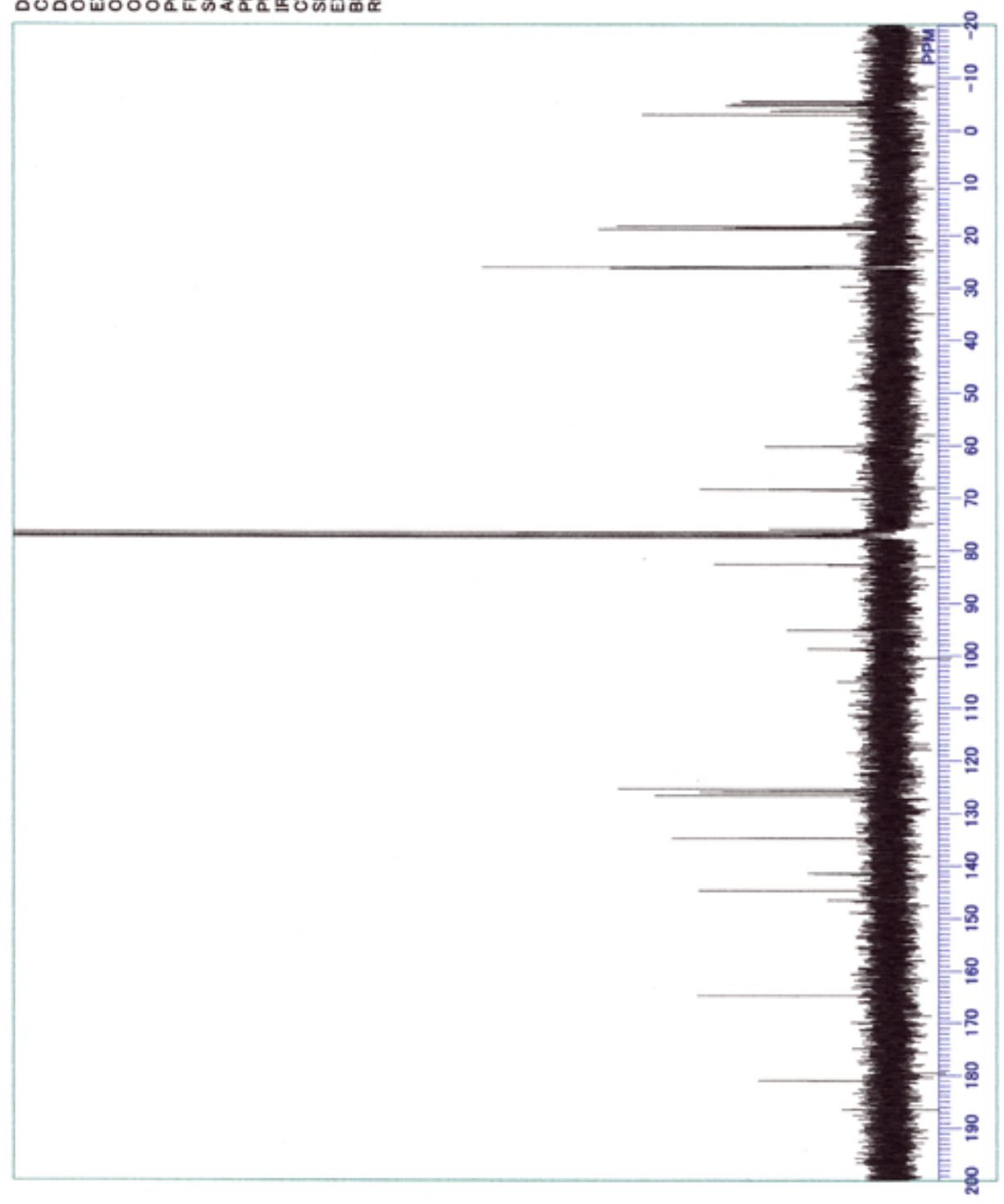




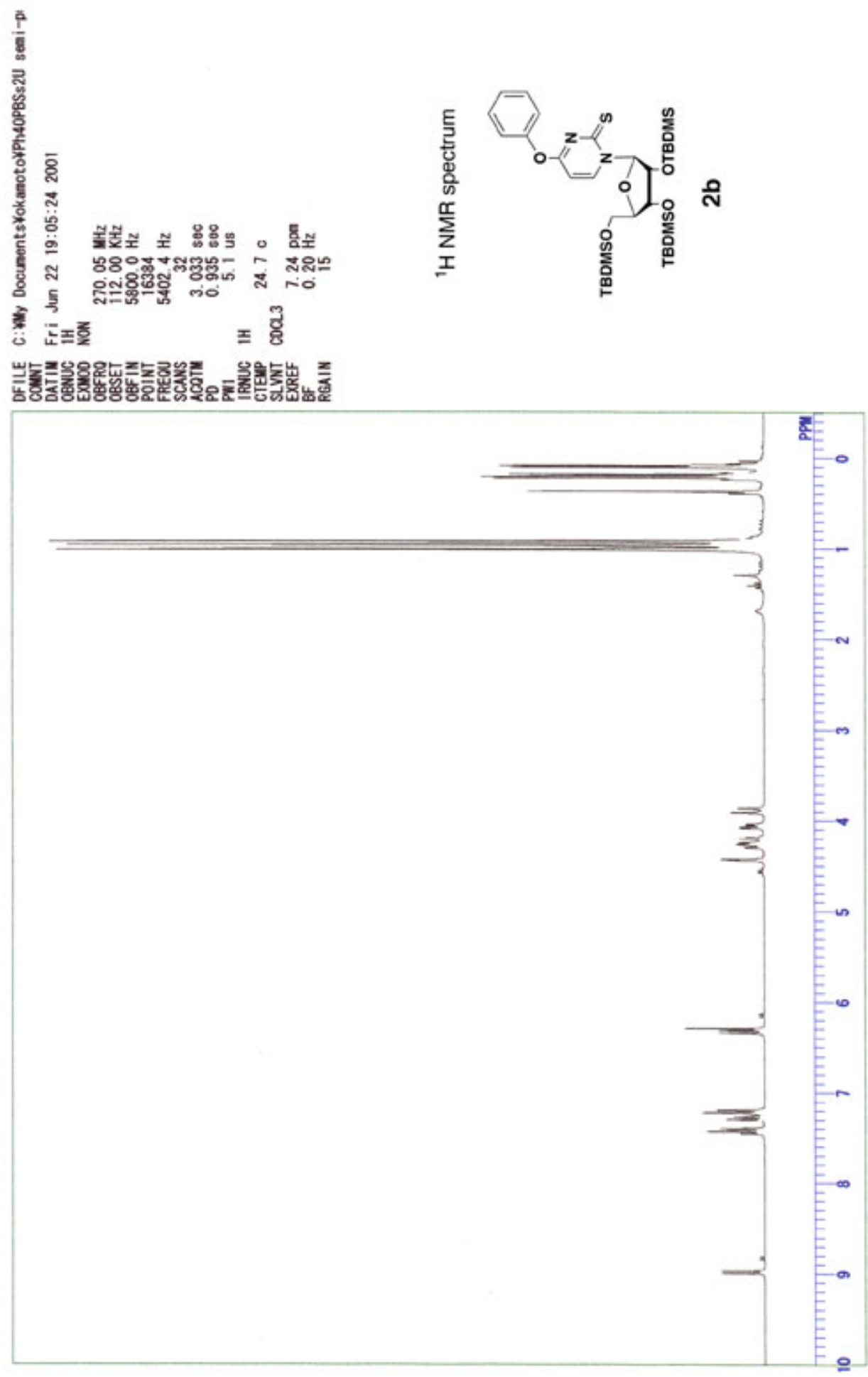



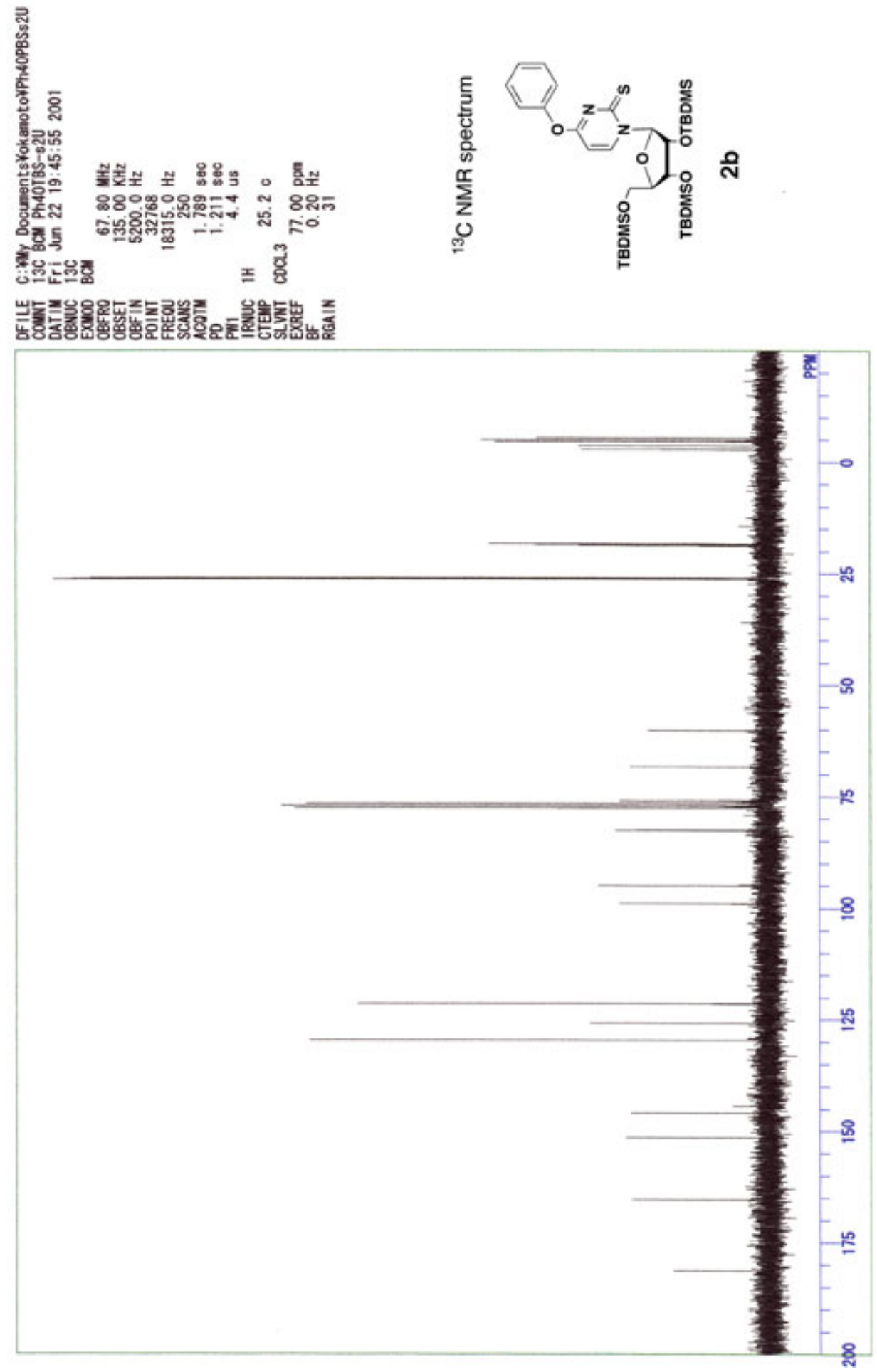

S16 


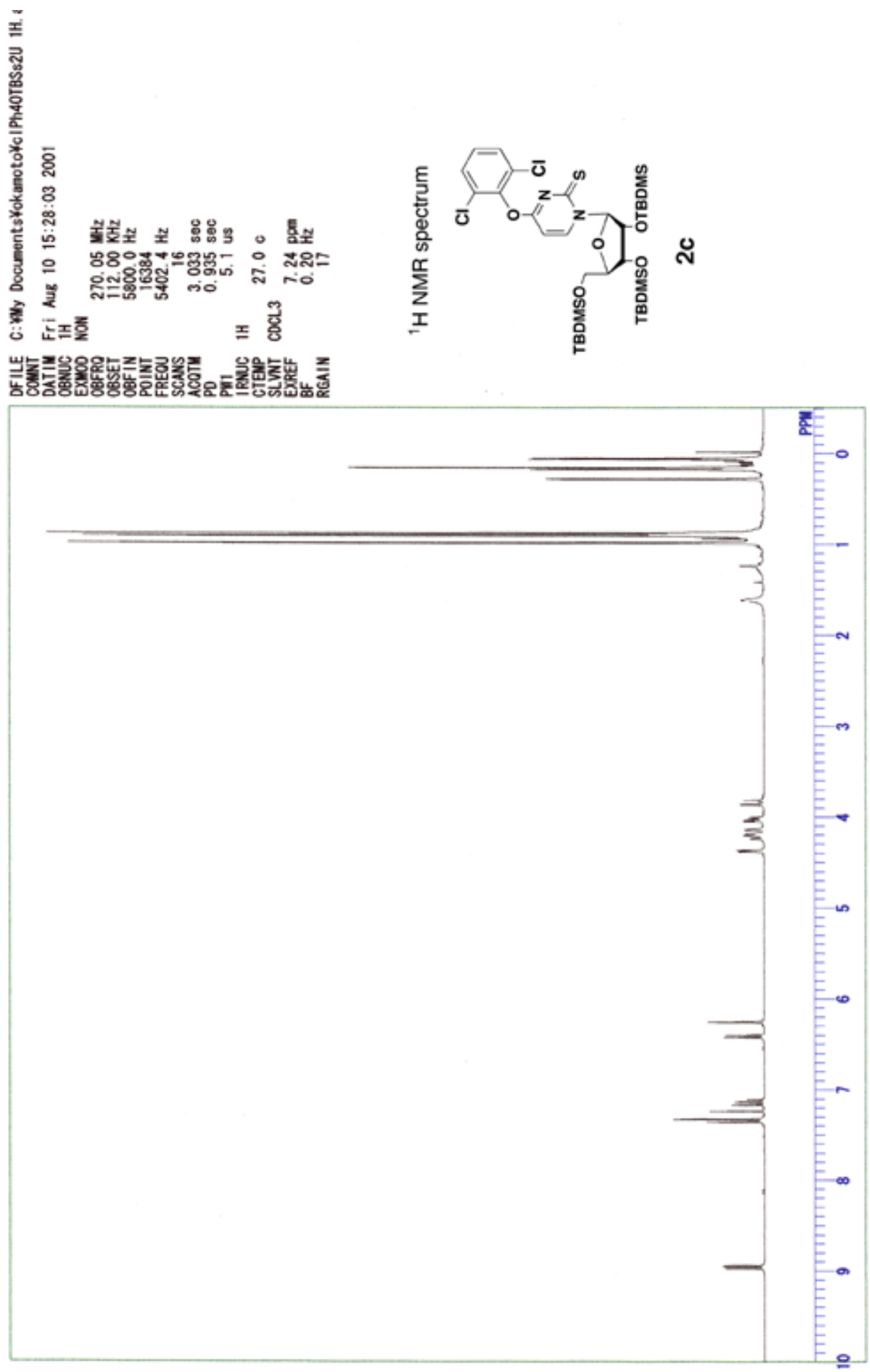



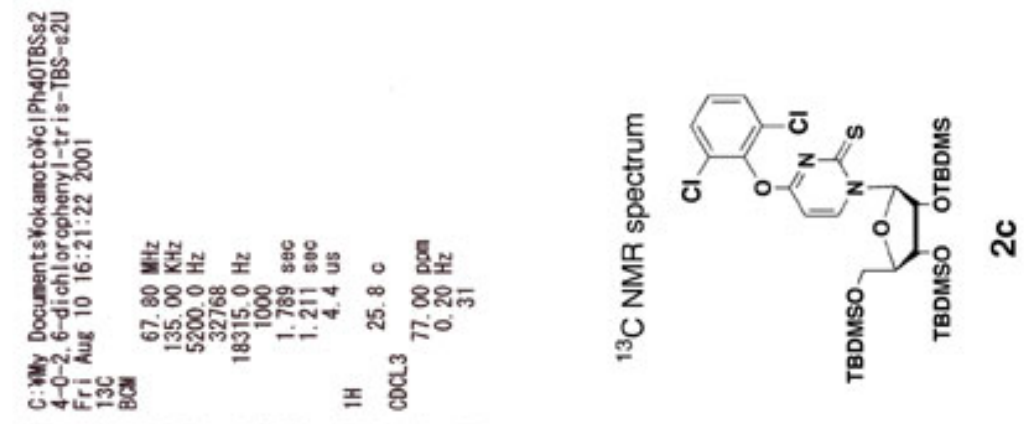

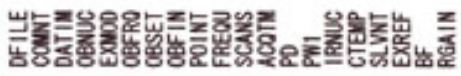

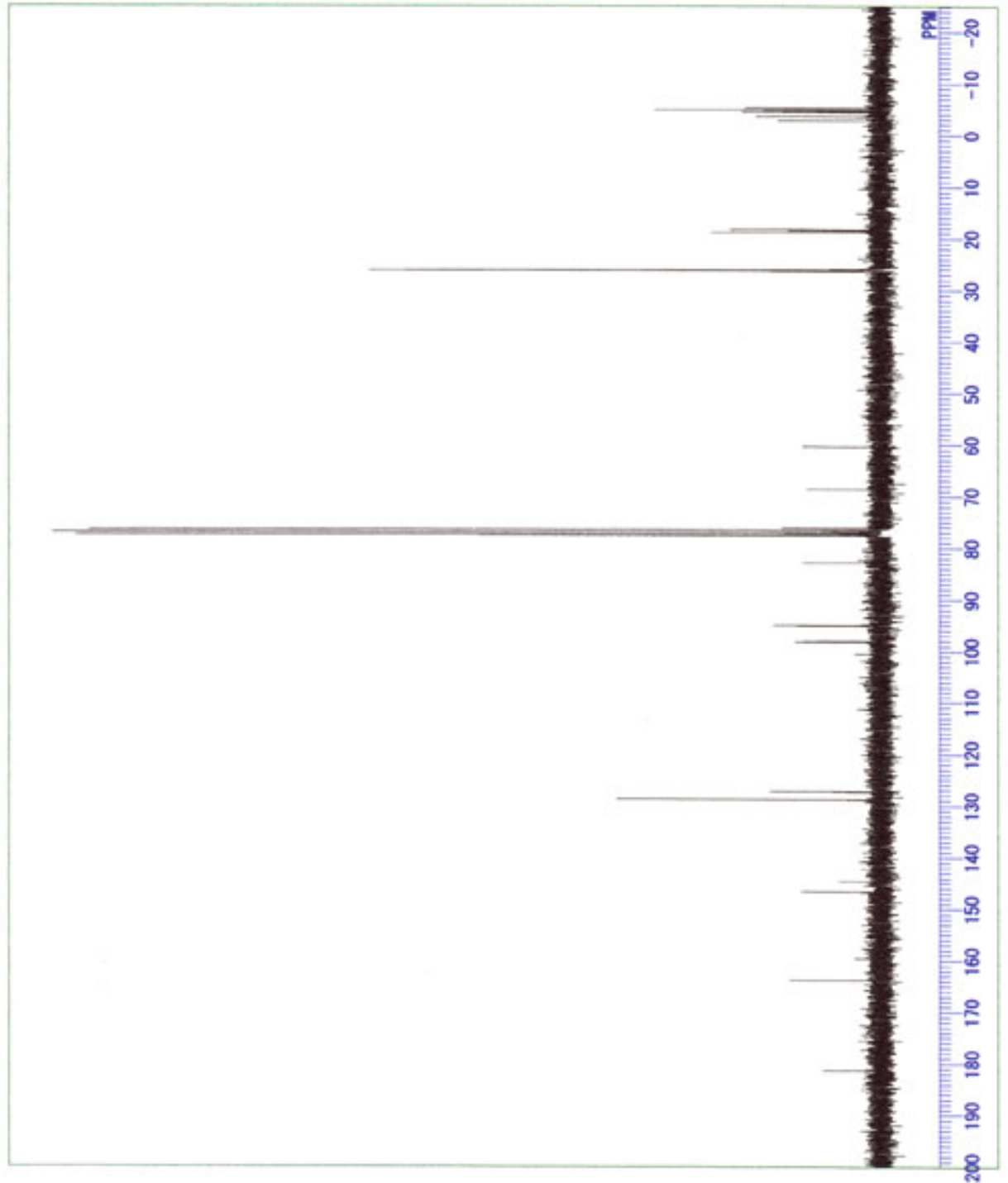




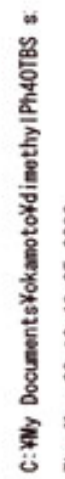

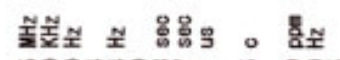

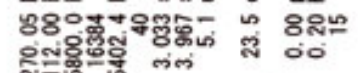
工

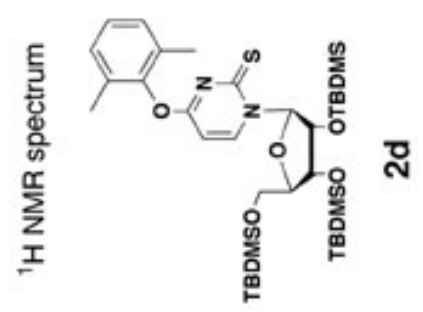

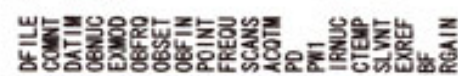

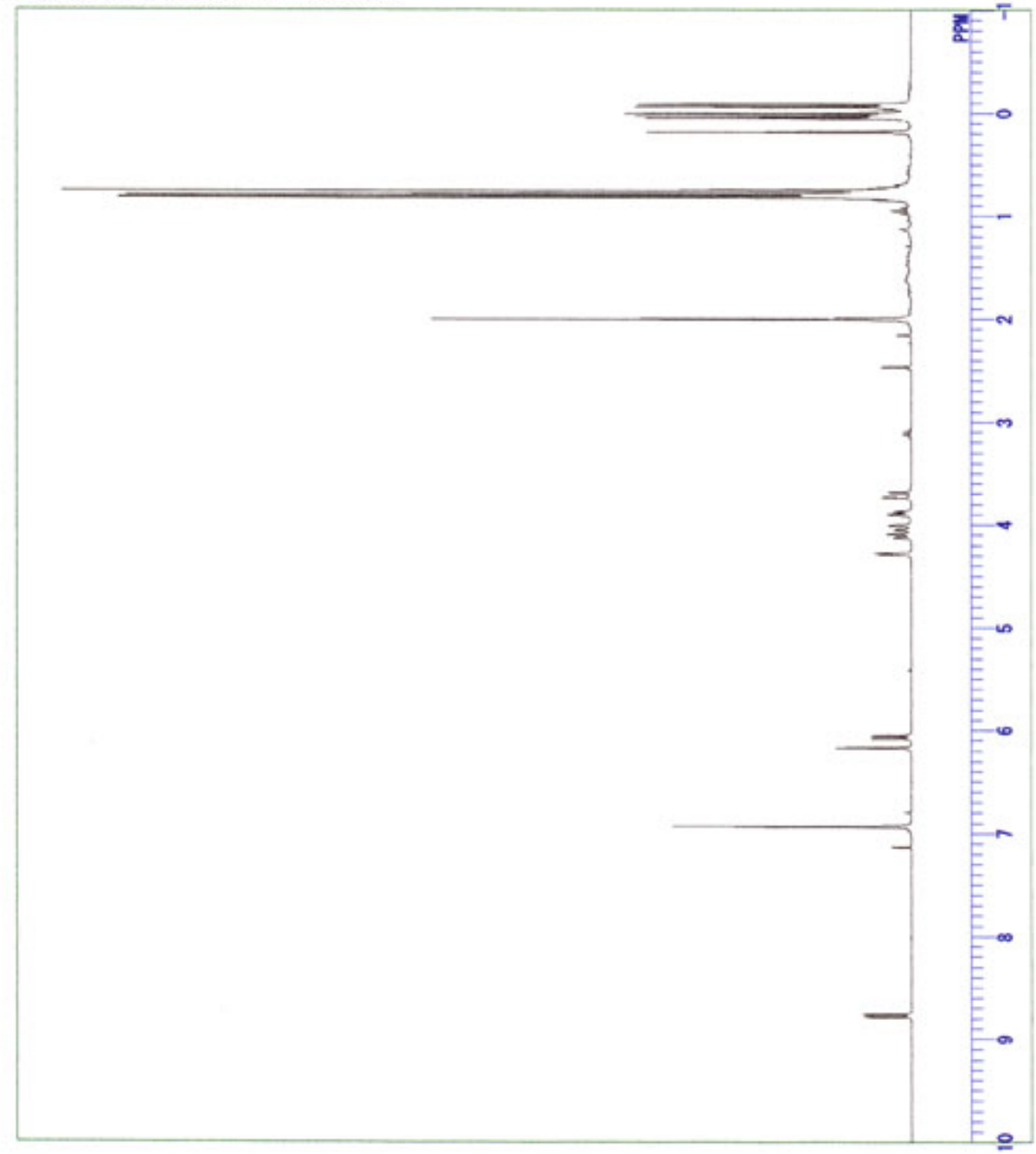

- 

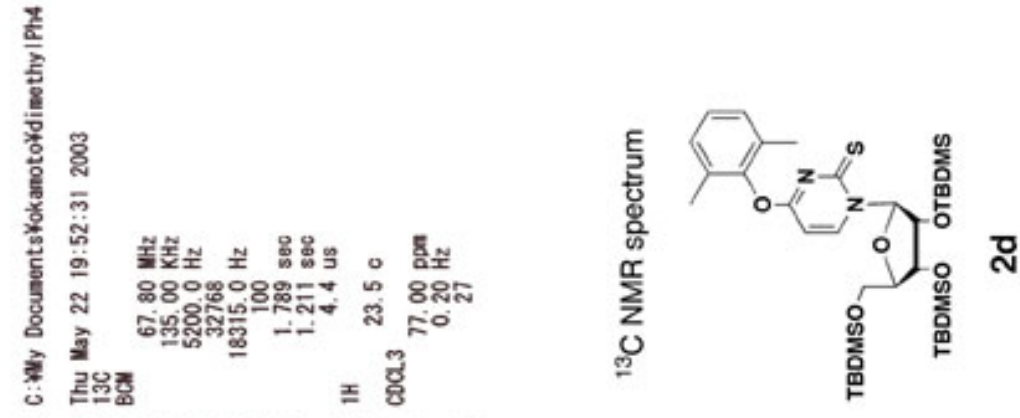

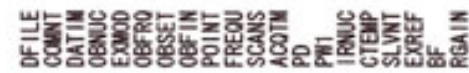

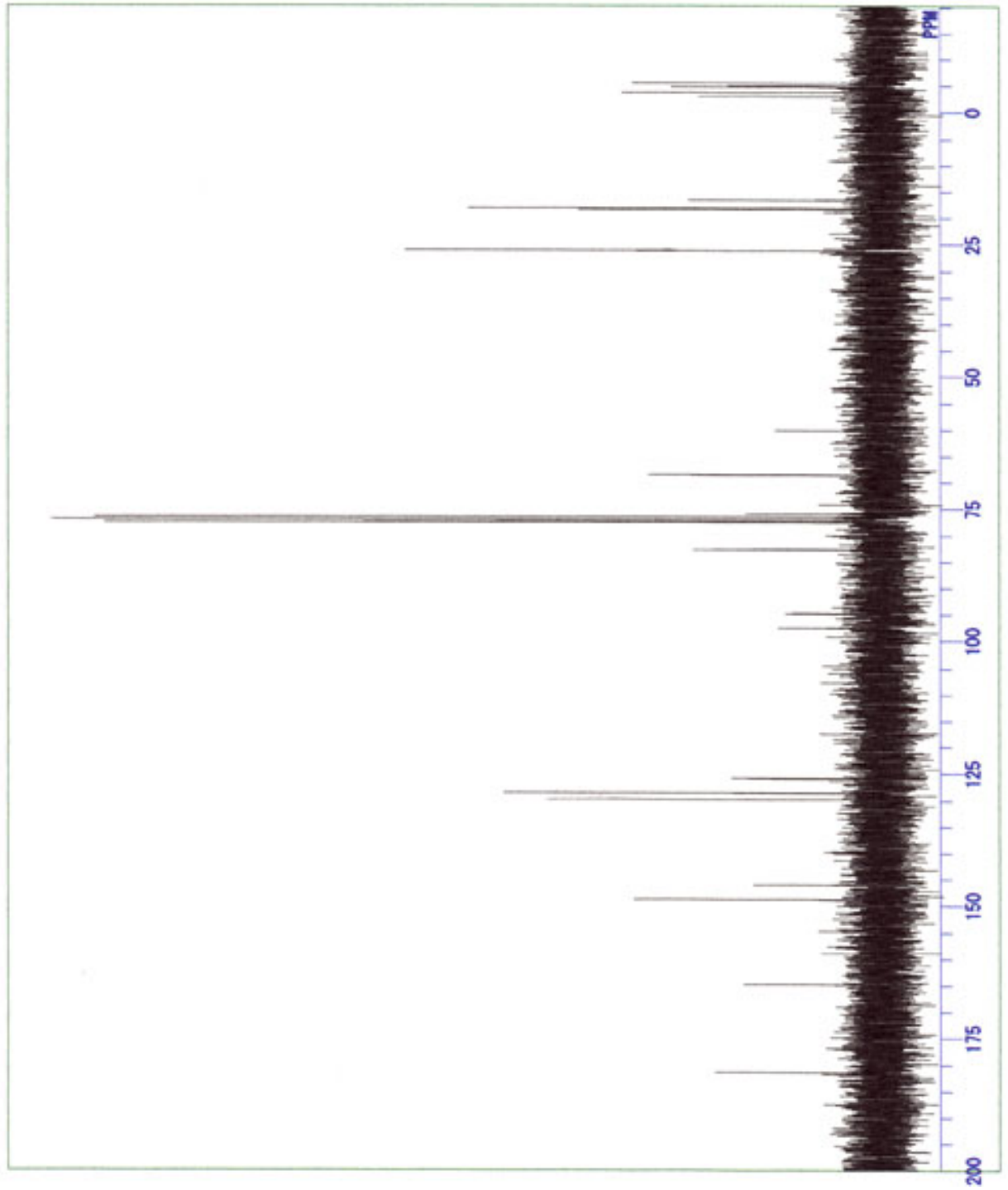




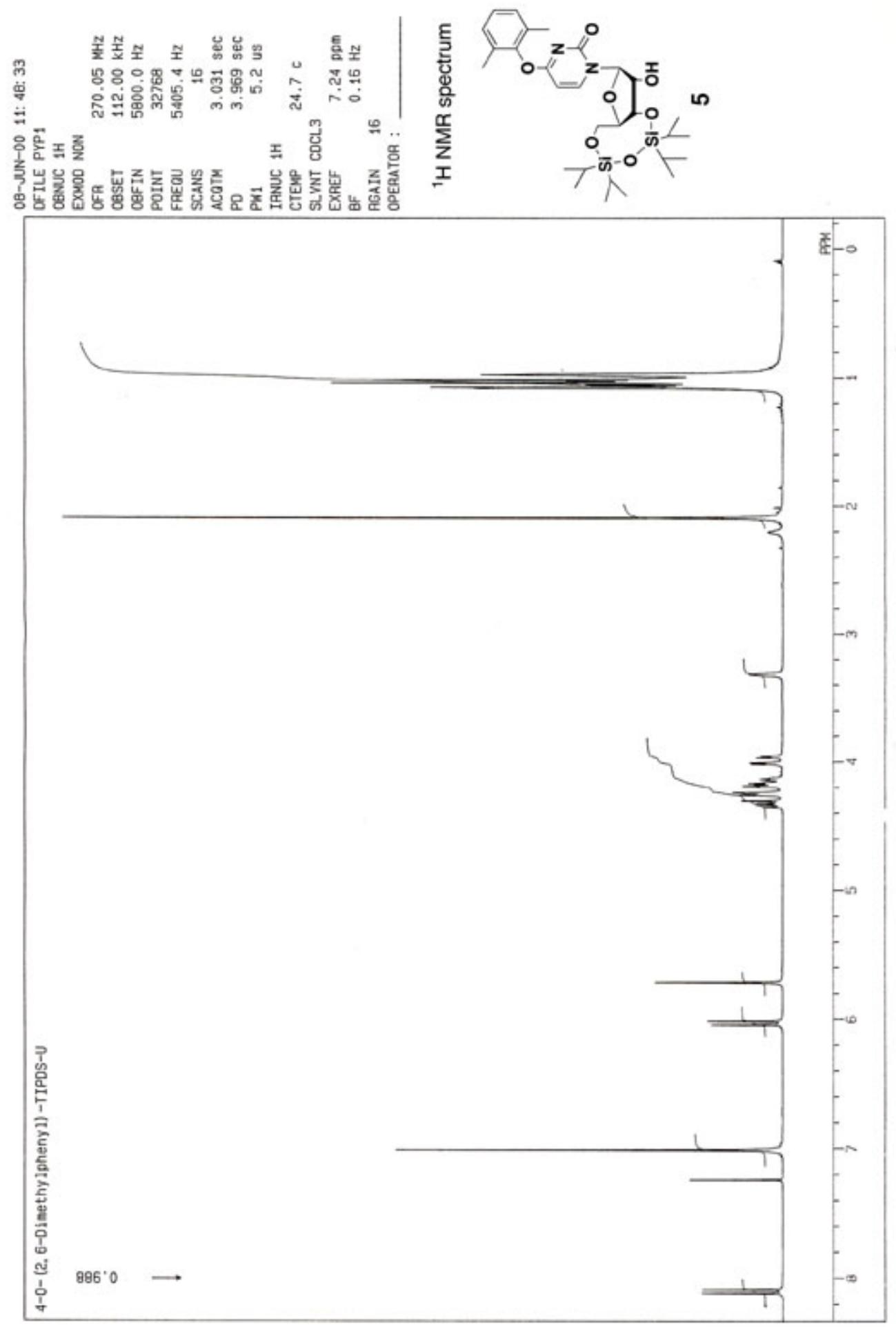

I 


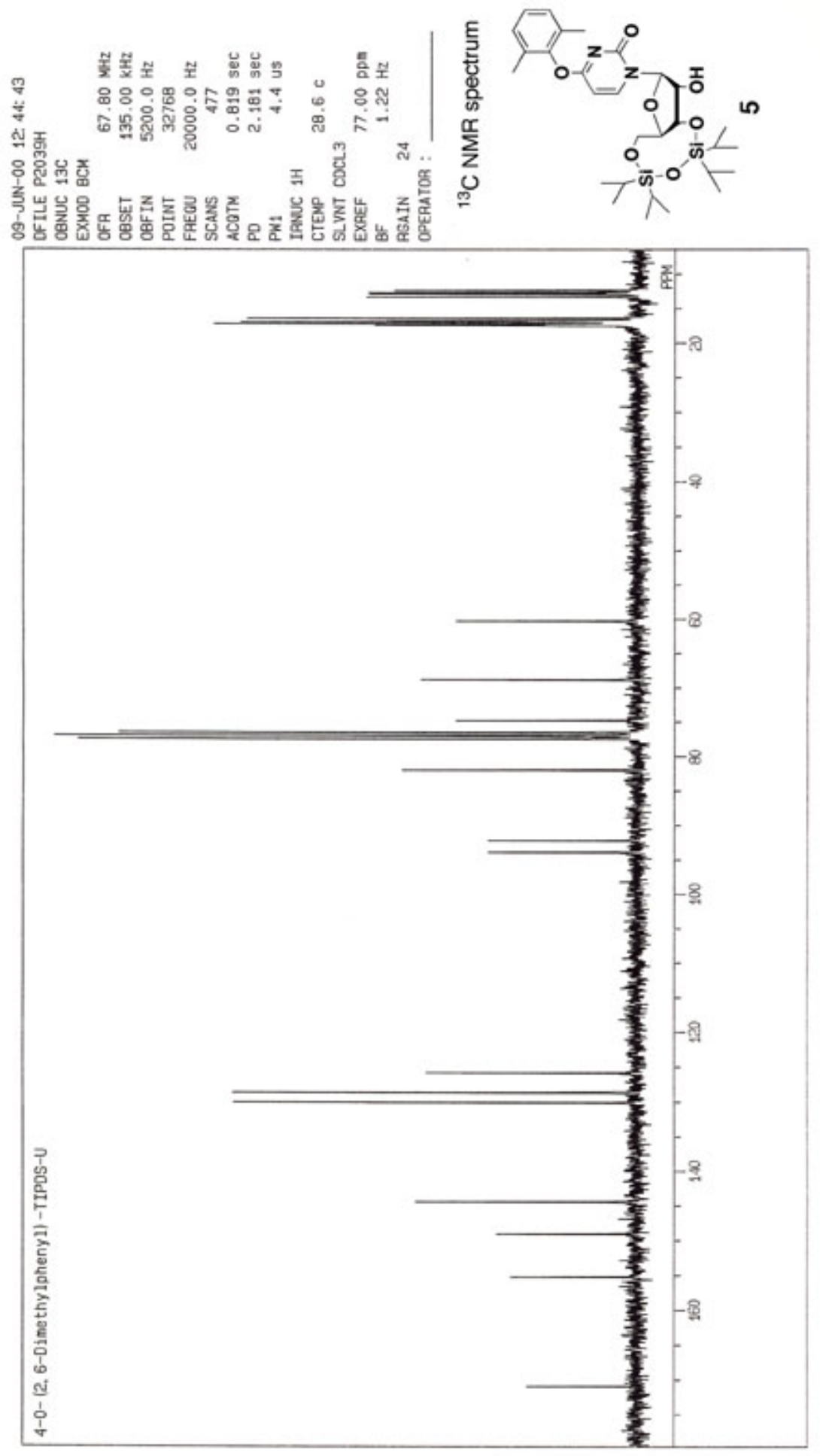




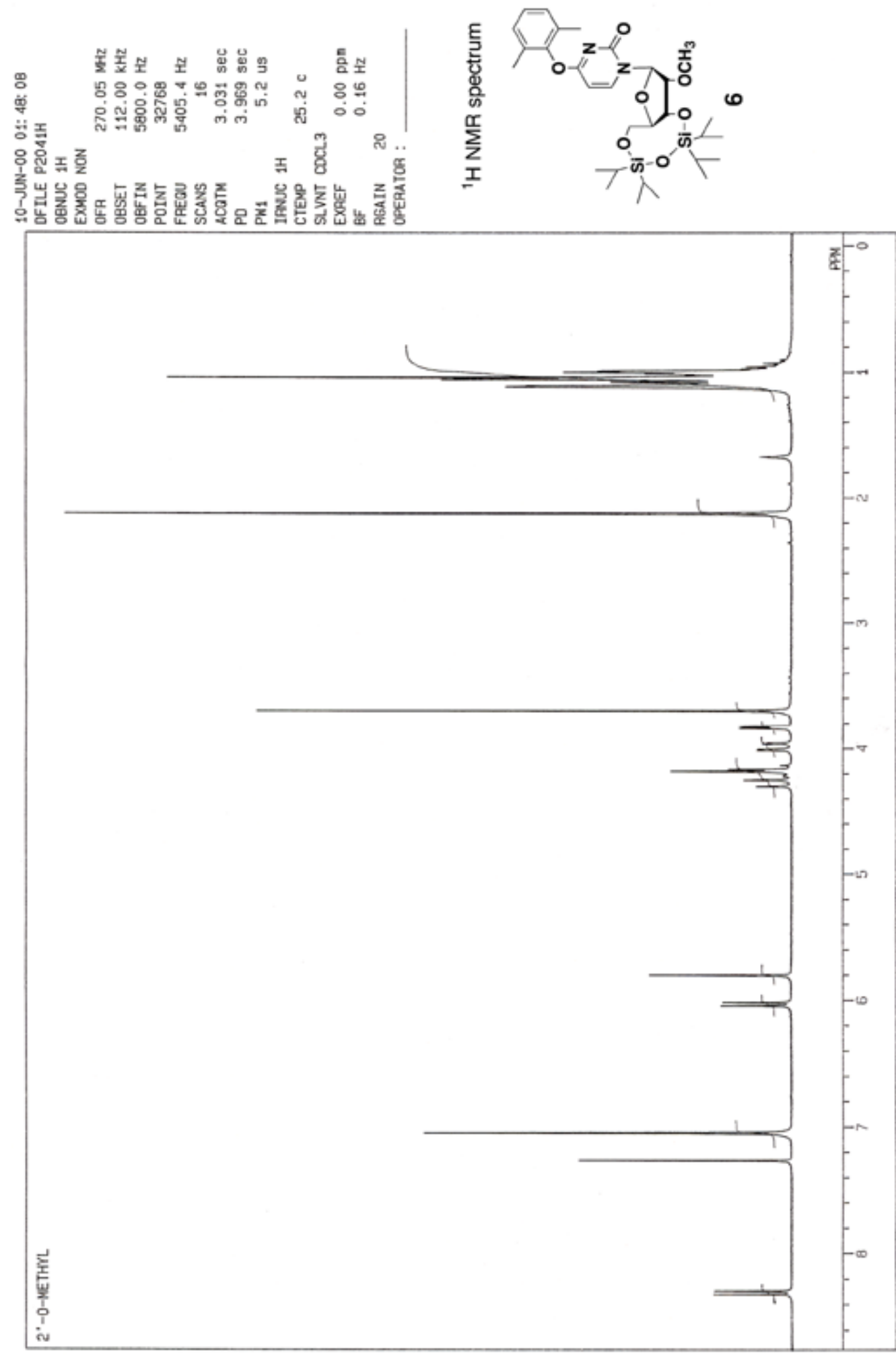




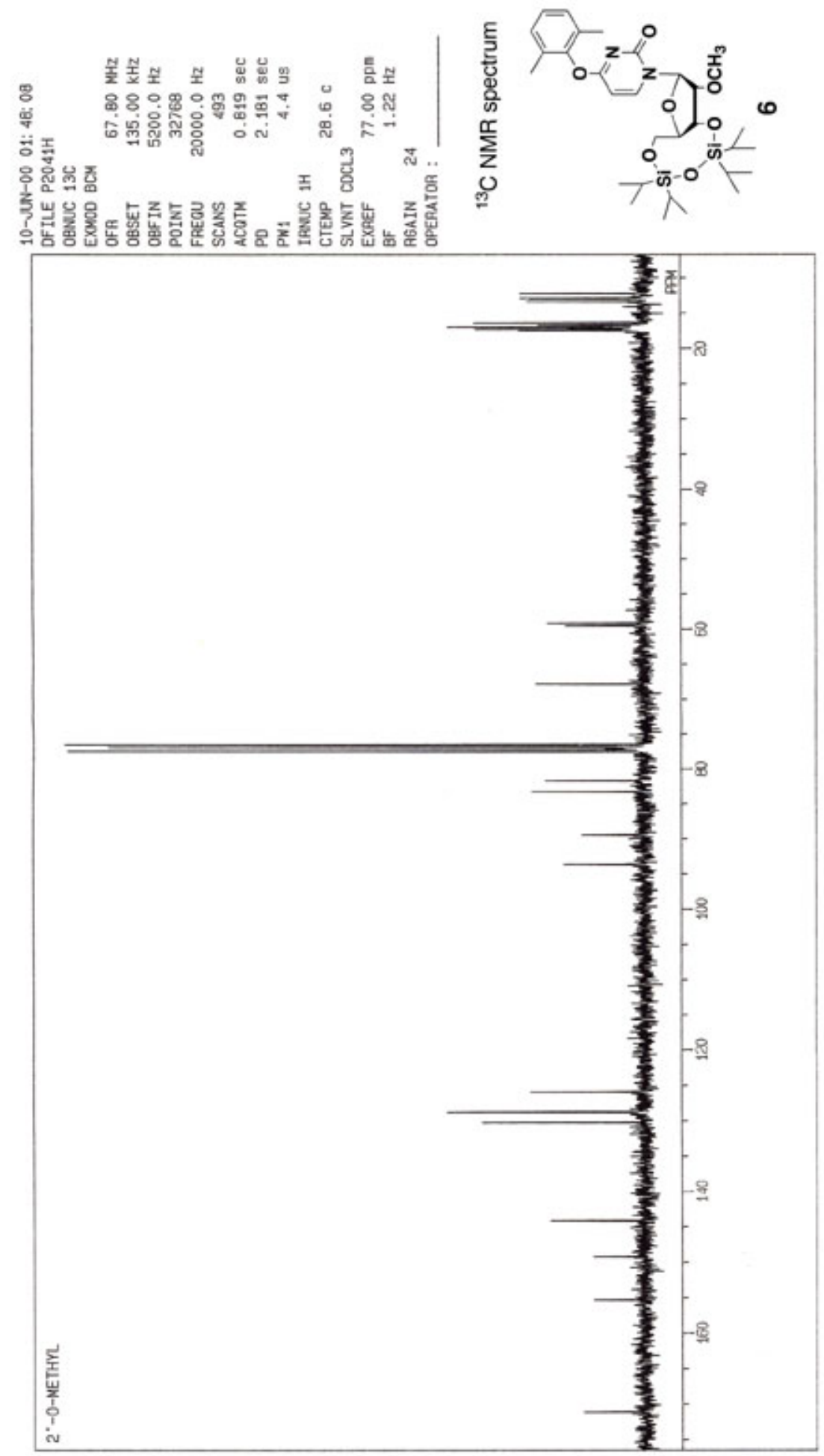




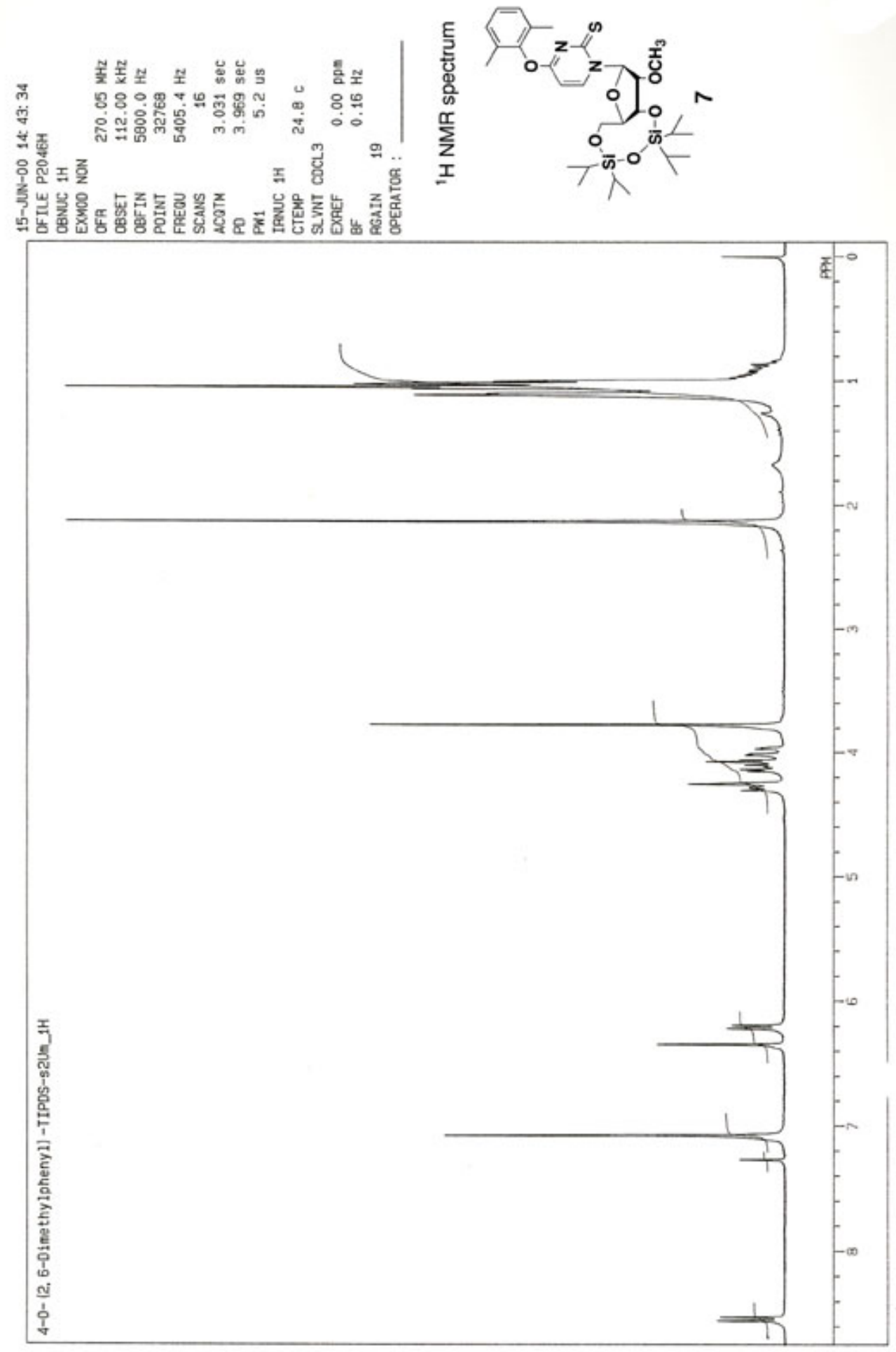




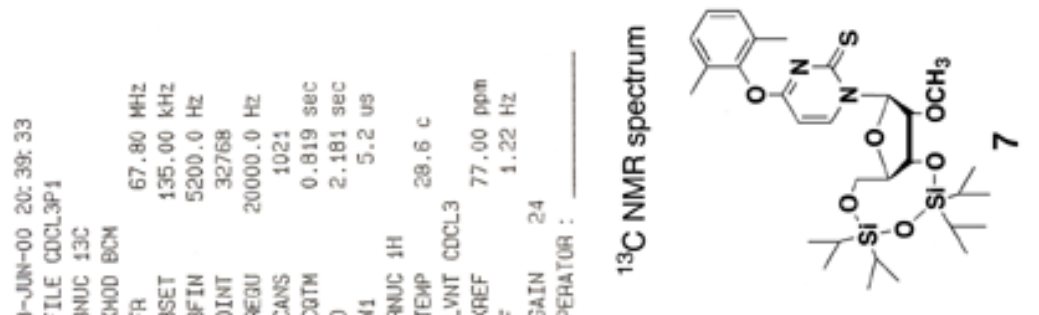

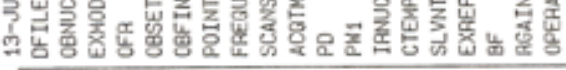

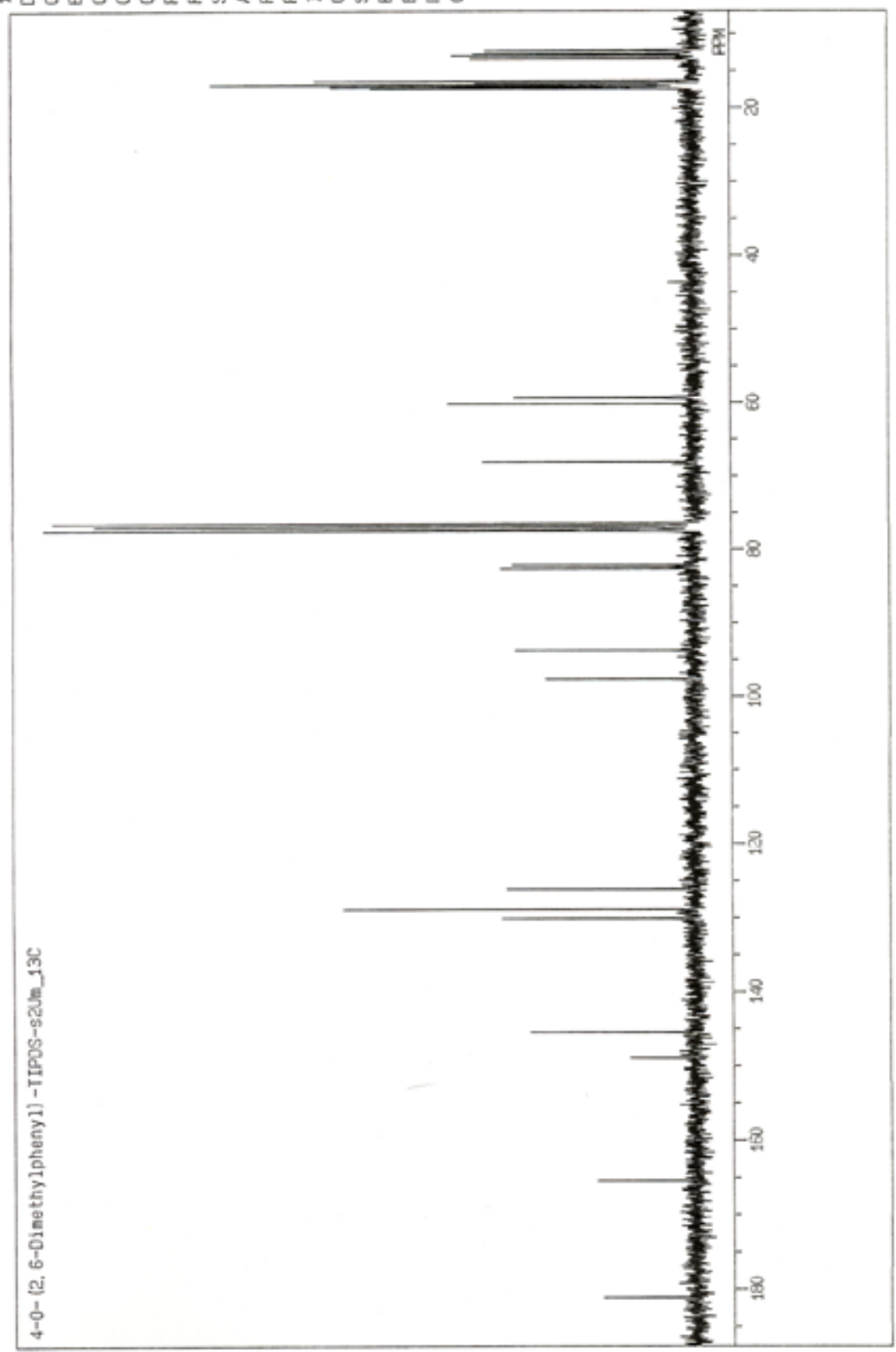




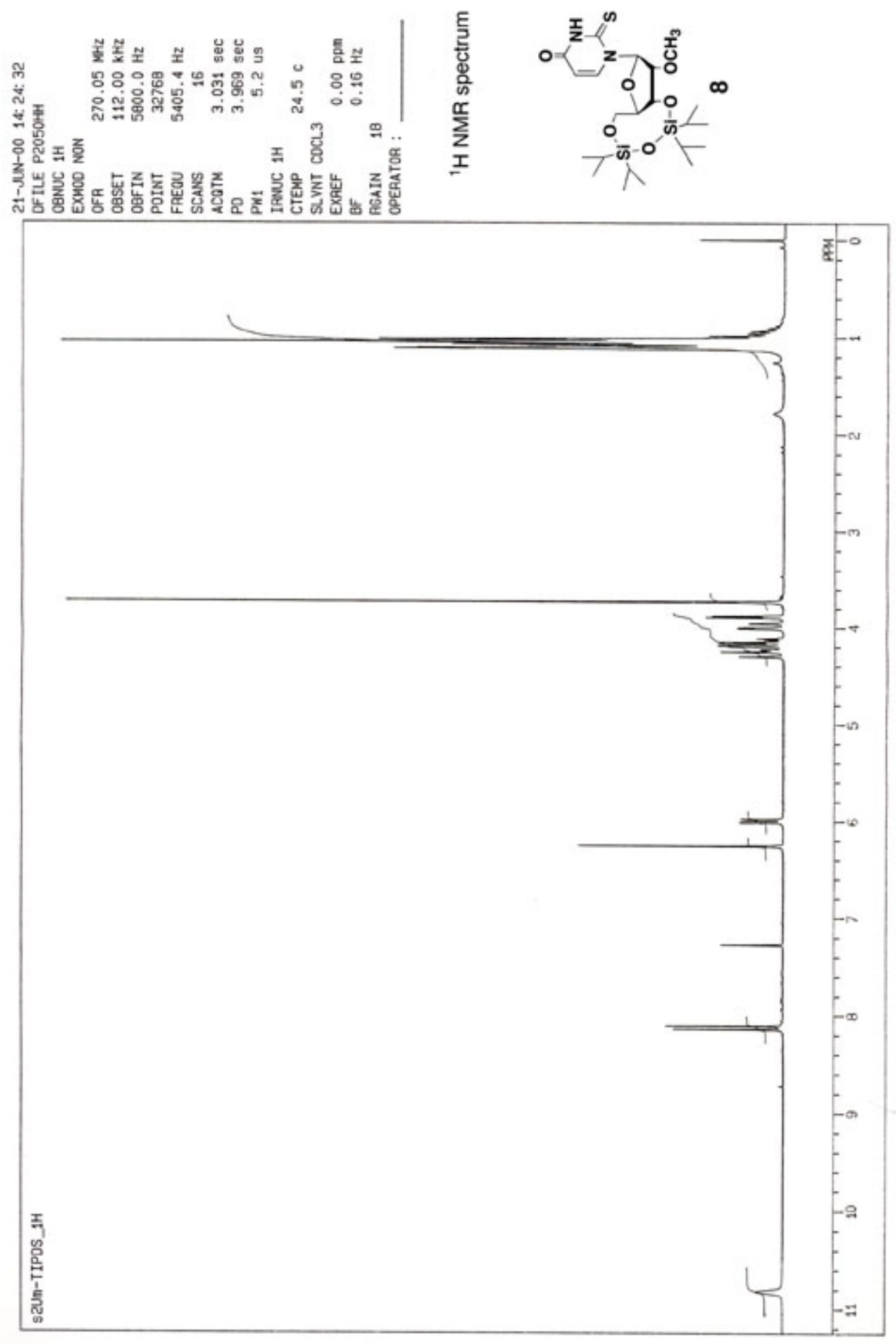




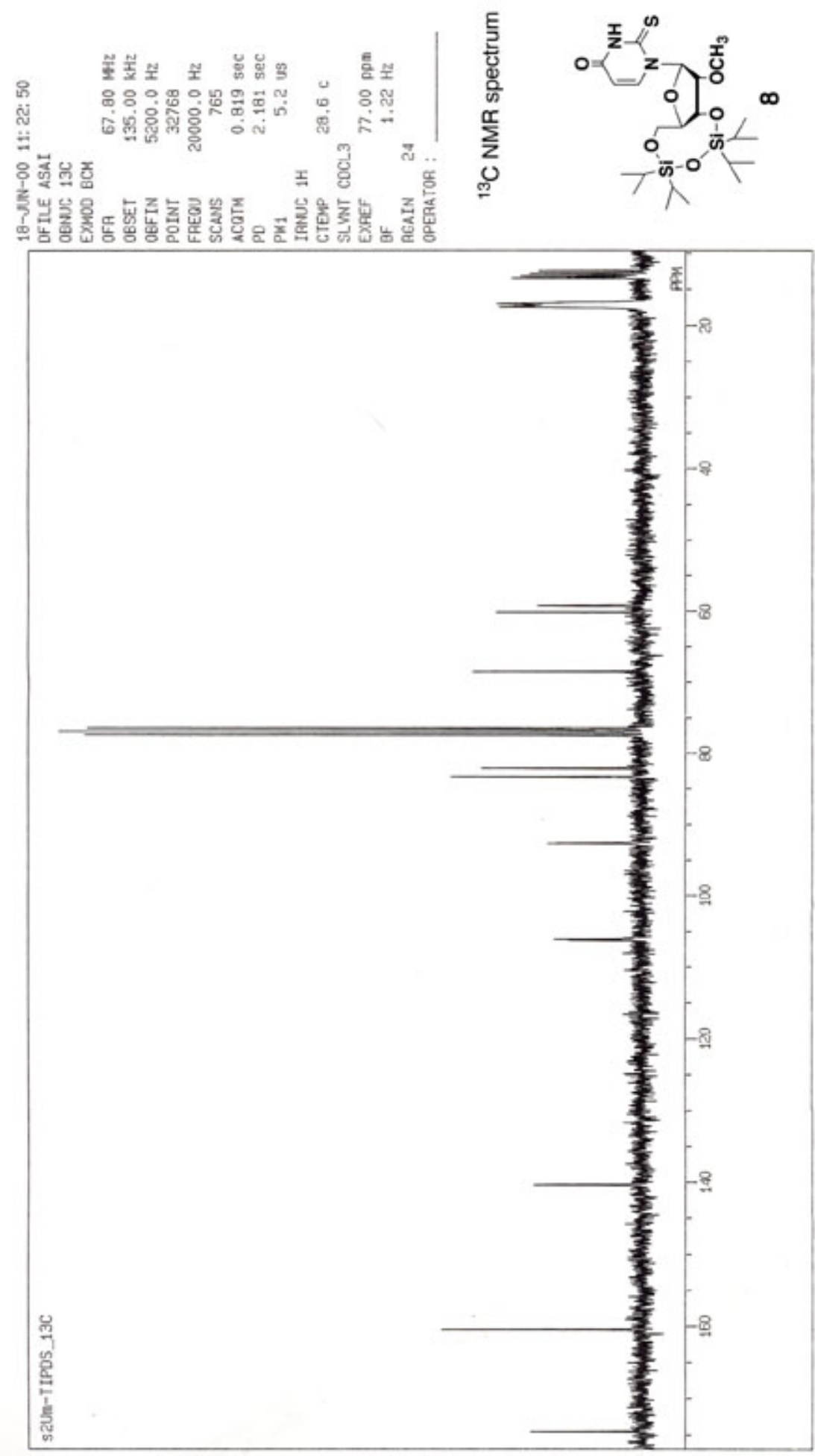




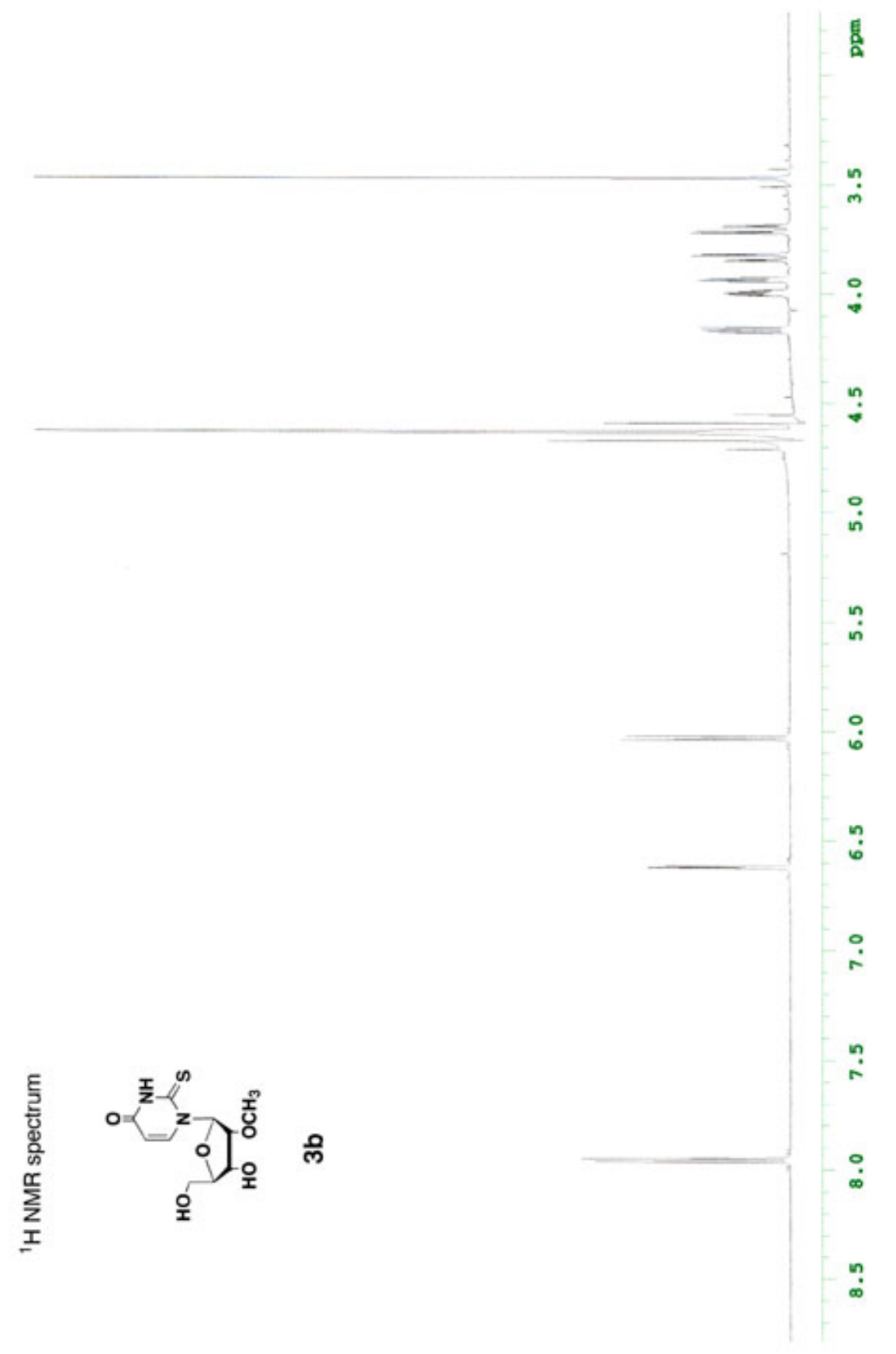




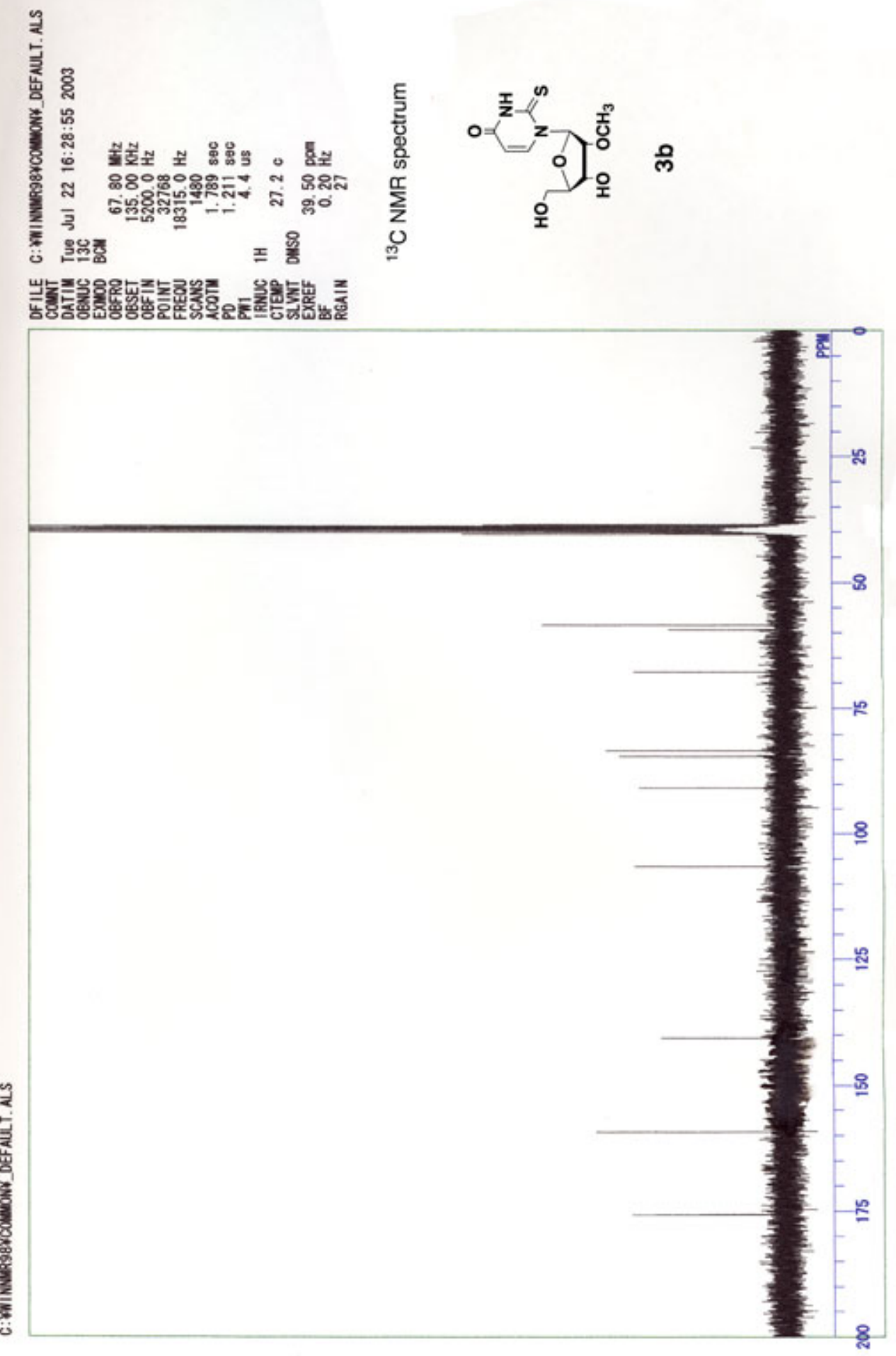




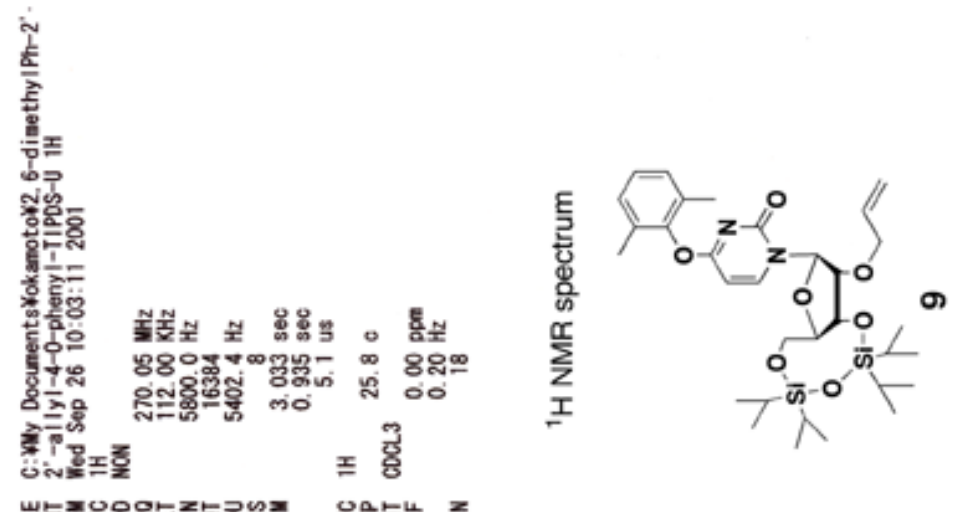

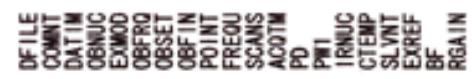

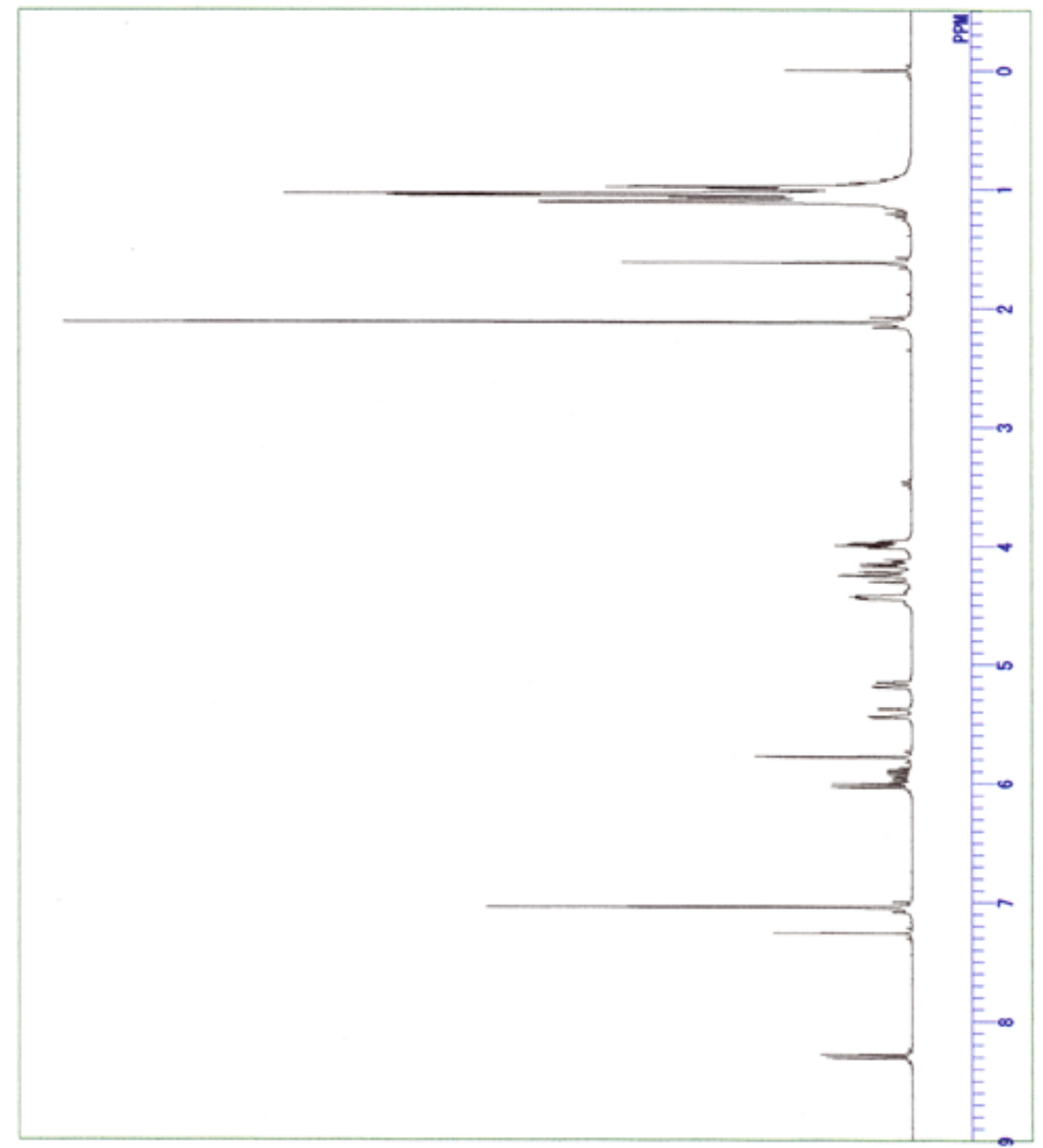




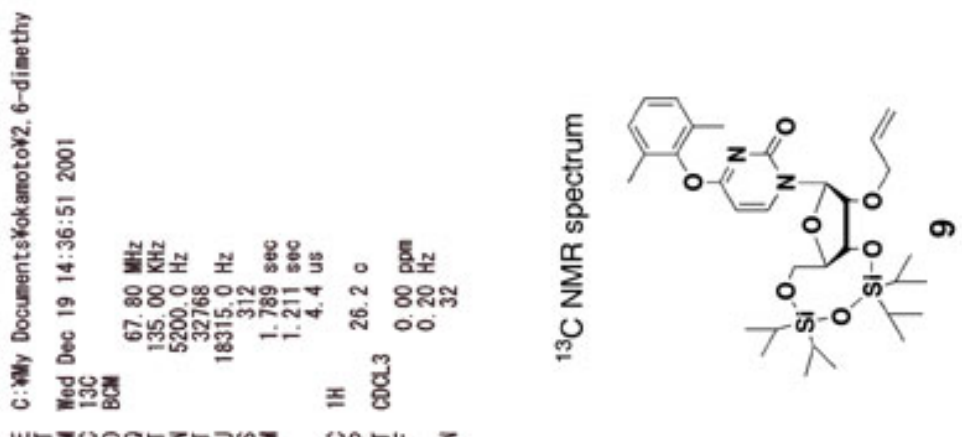

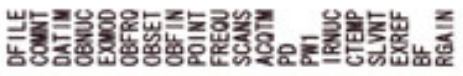

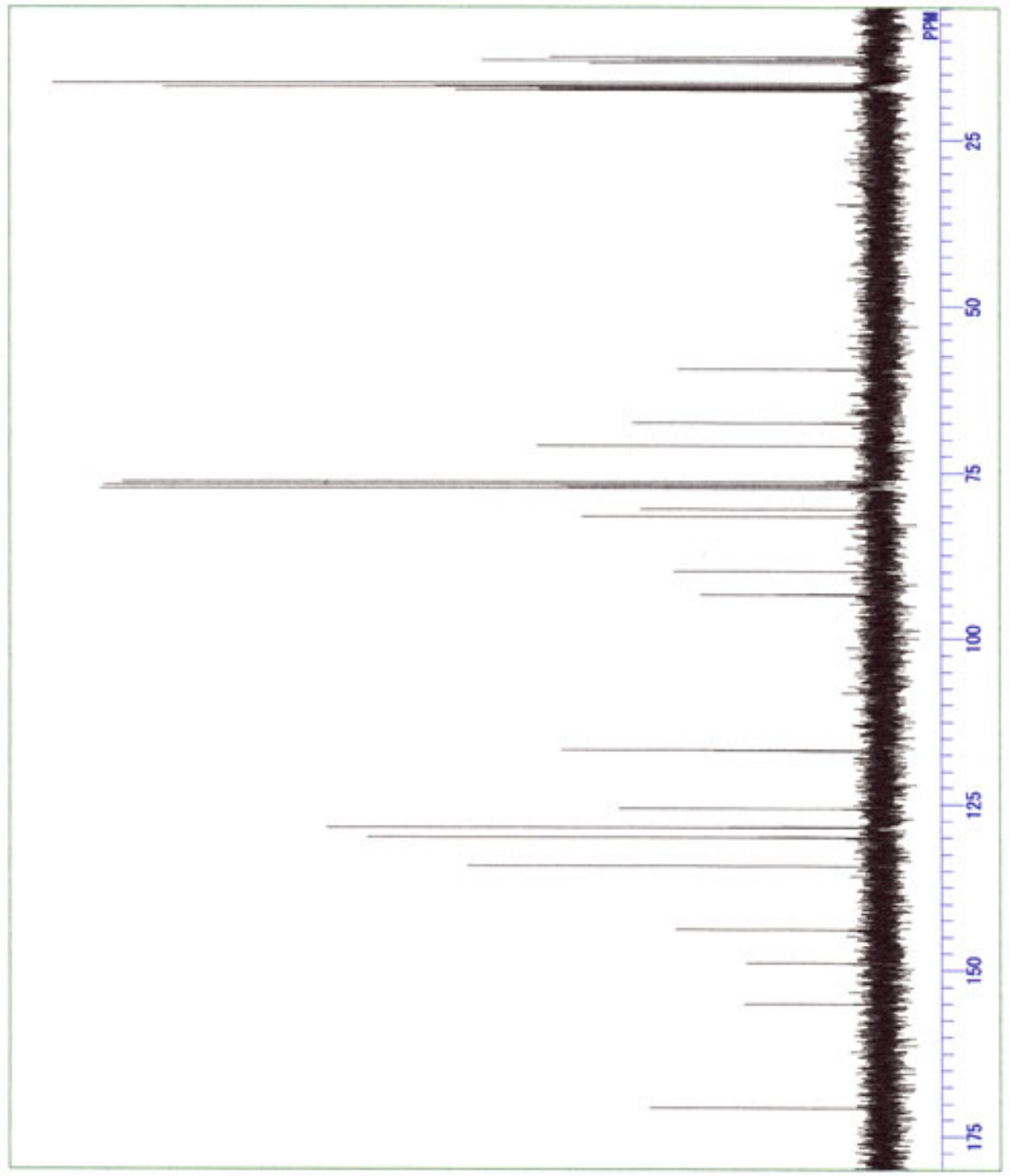




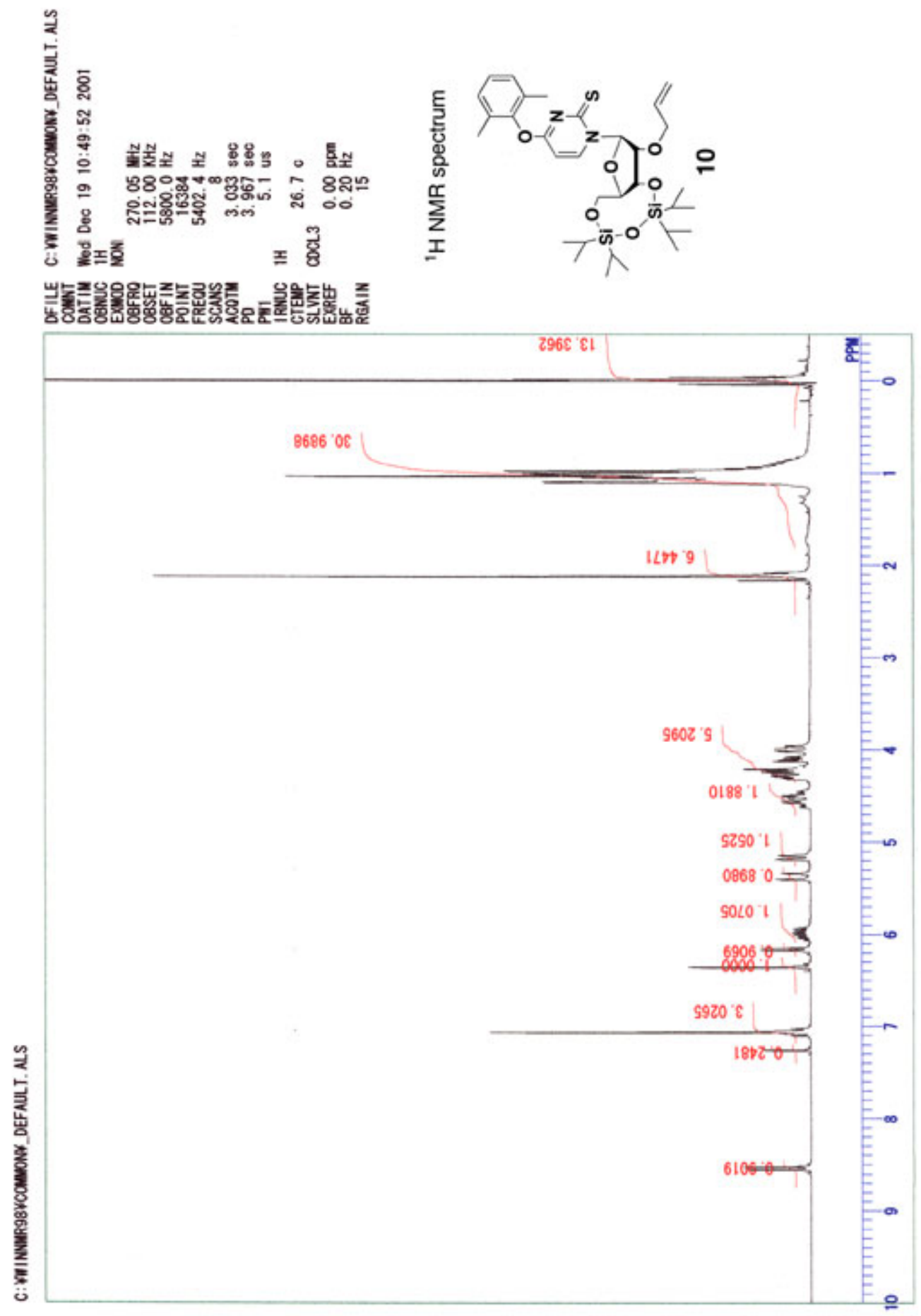




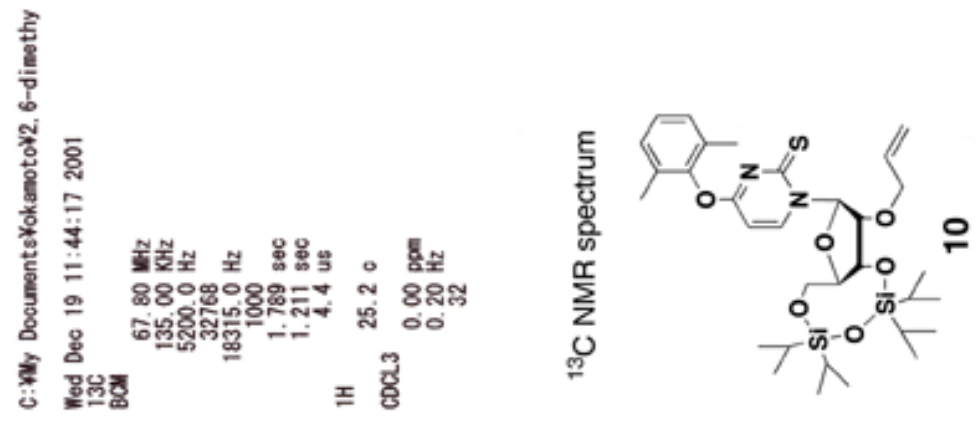

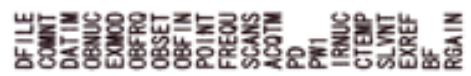

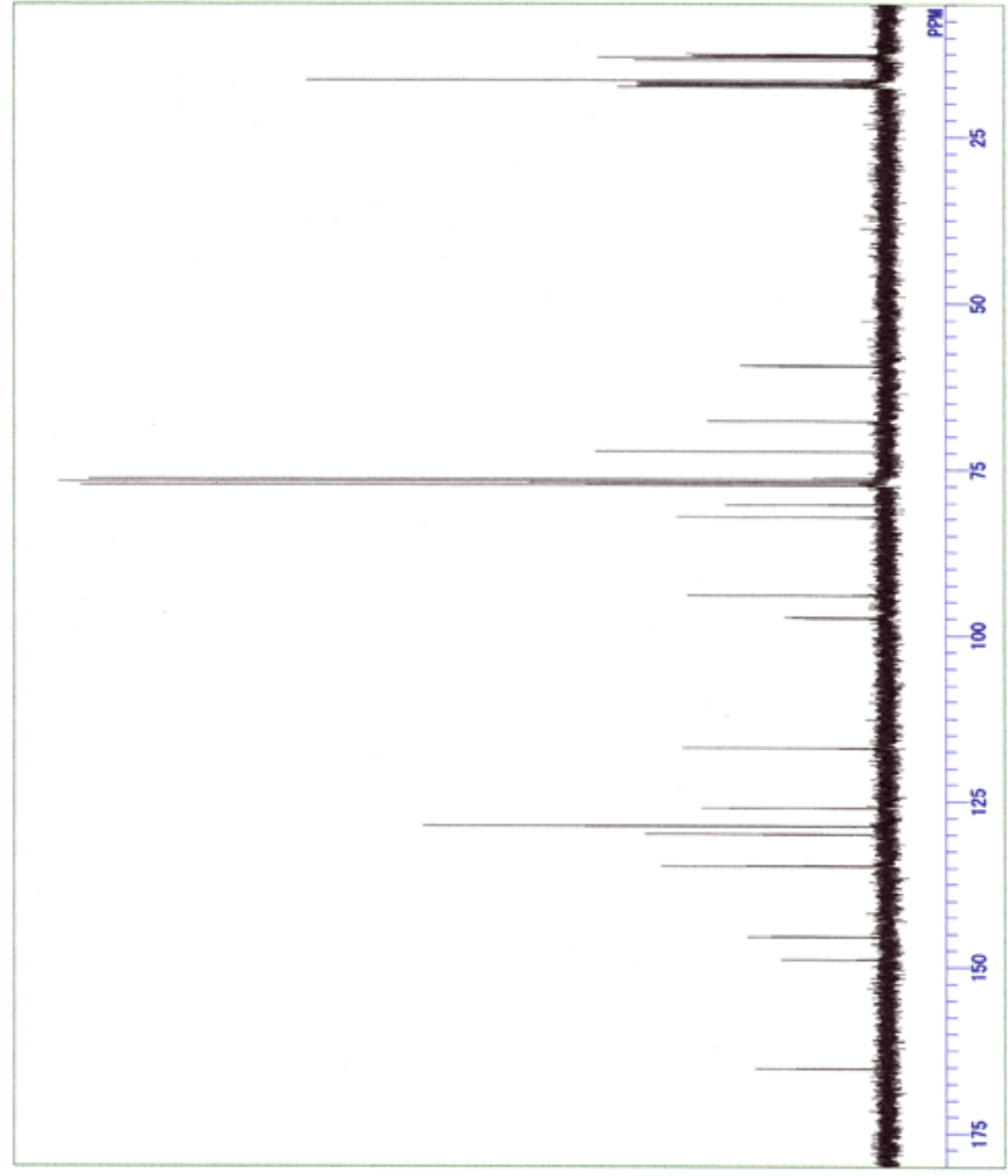




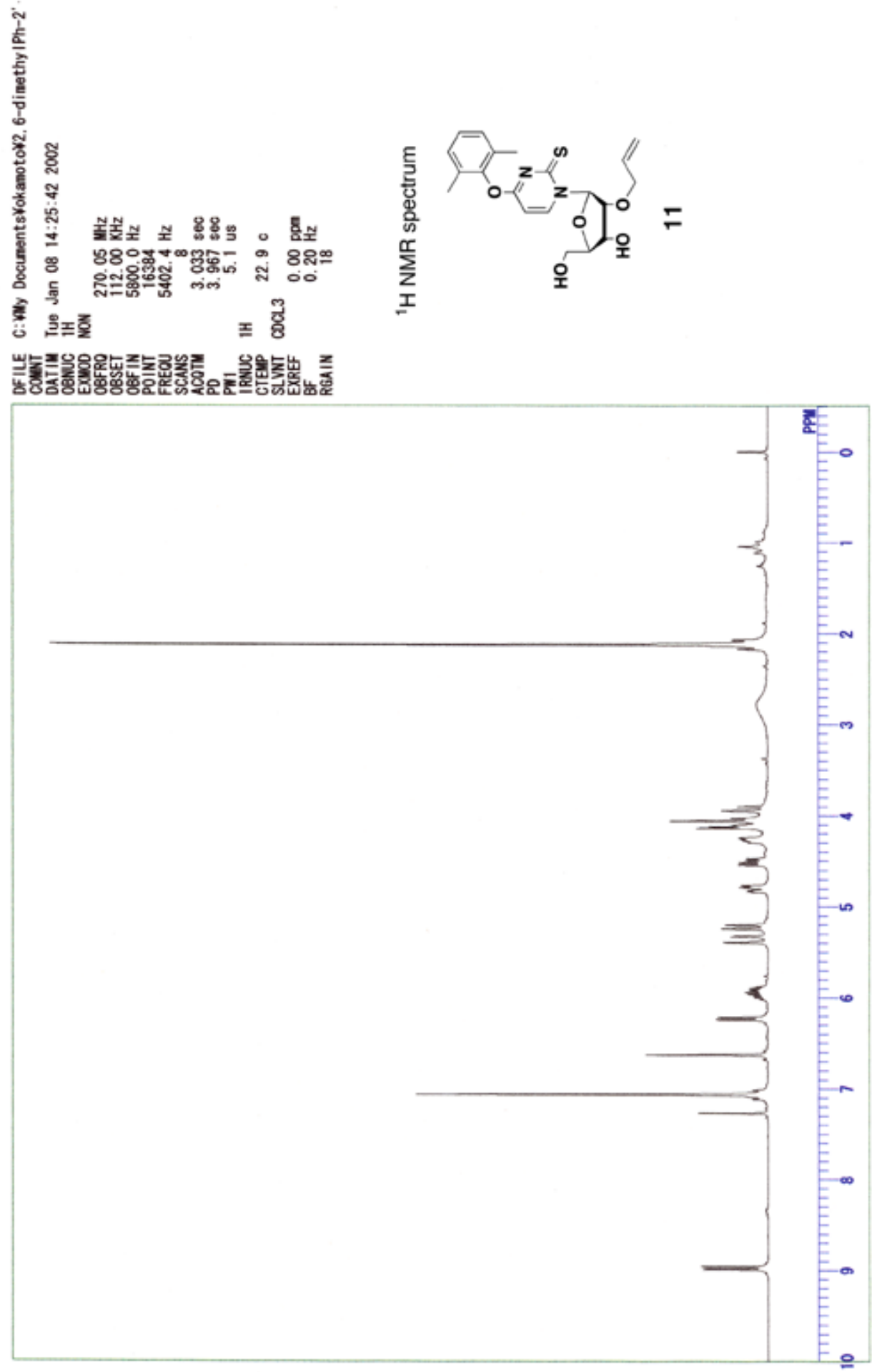




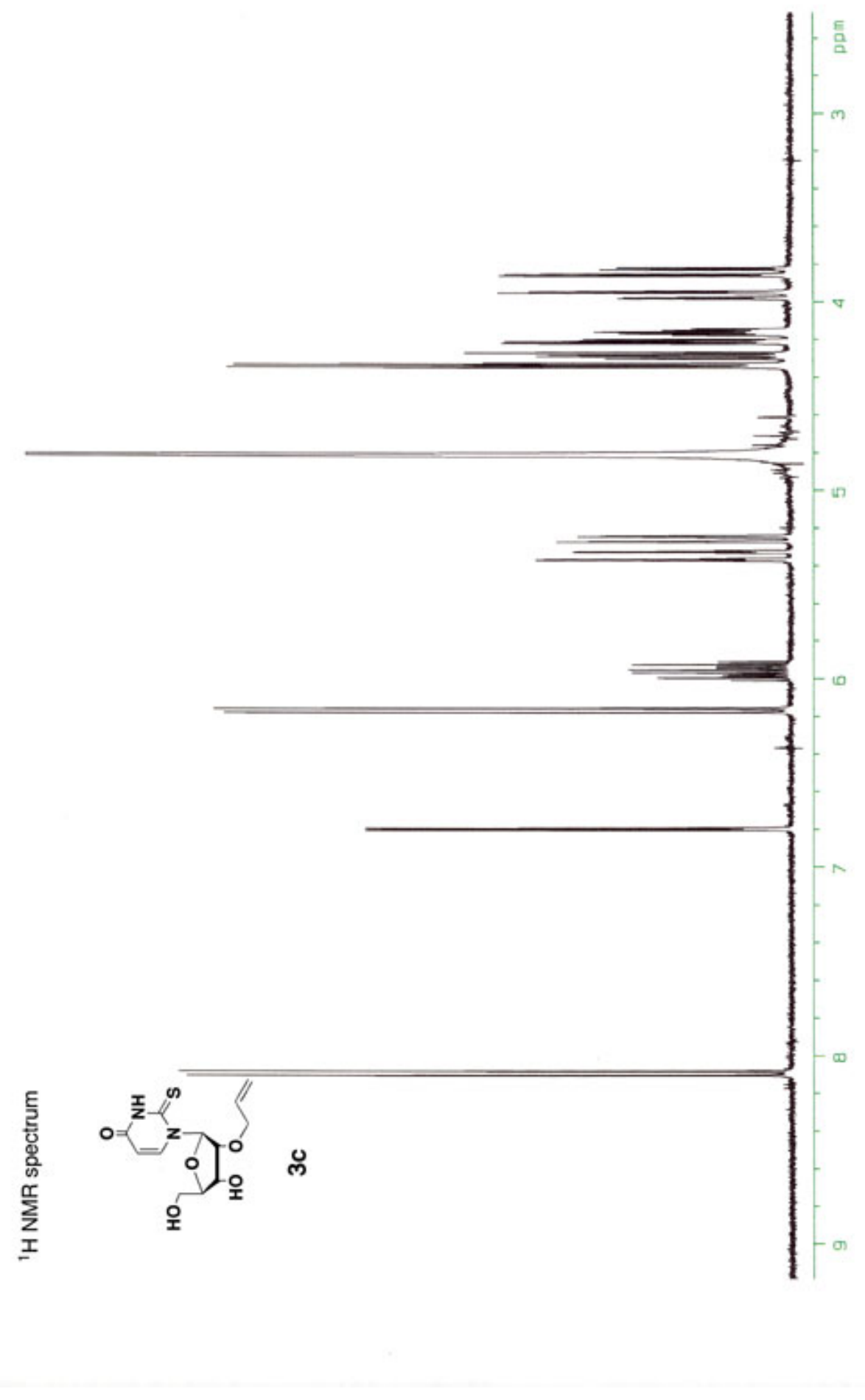




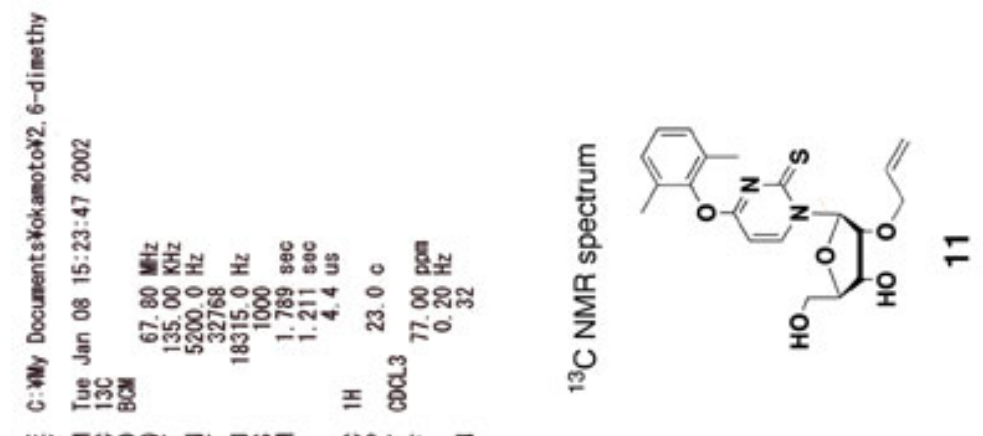

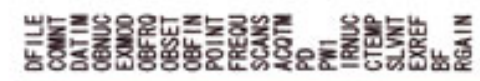

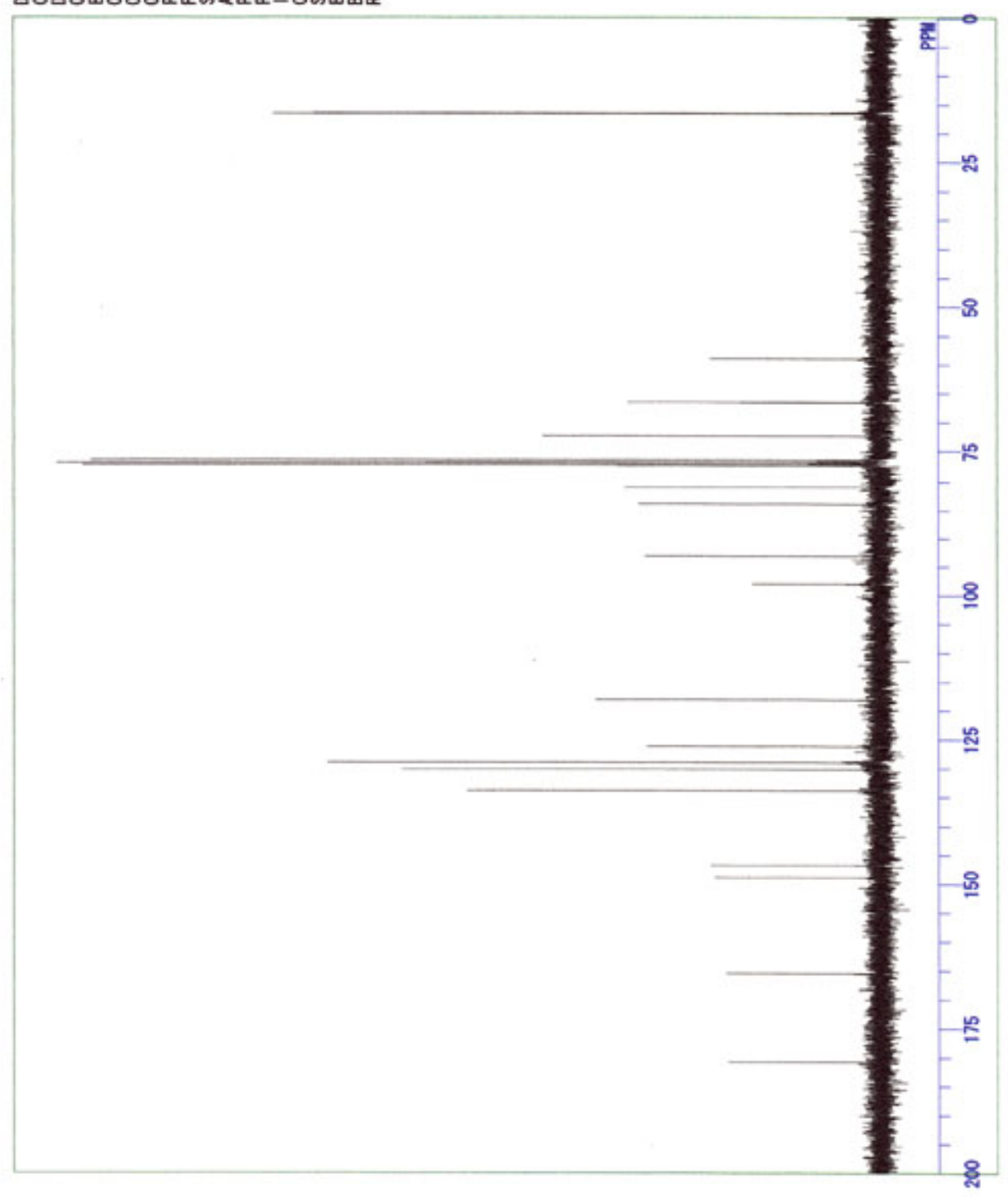




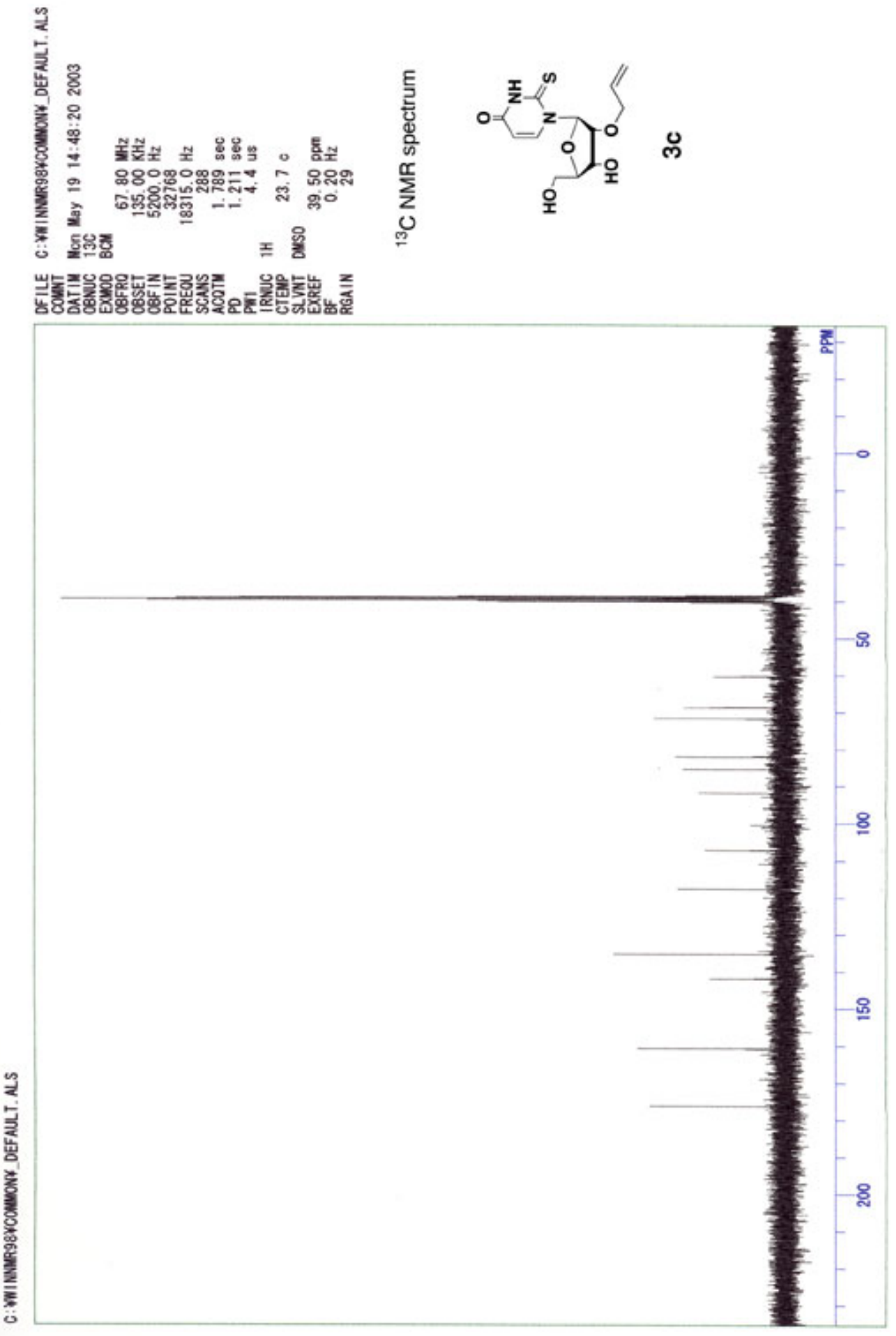




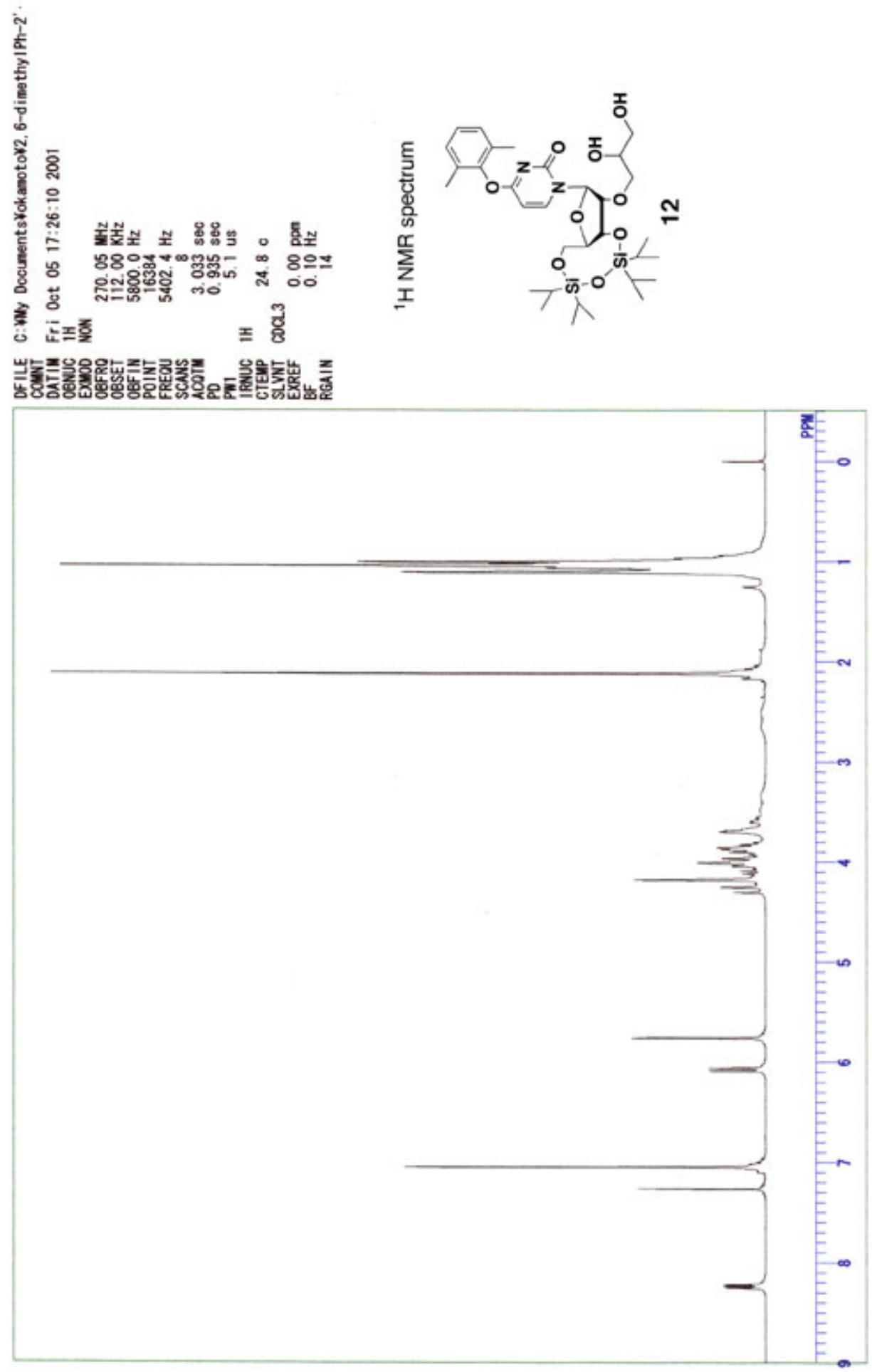

I 


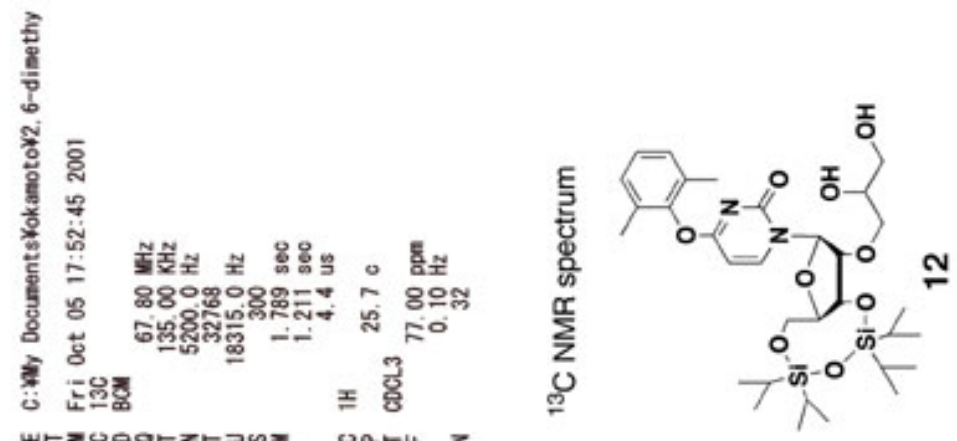

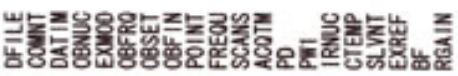

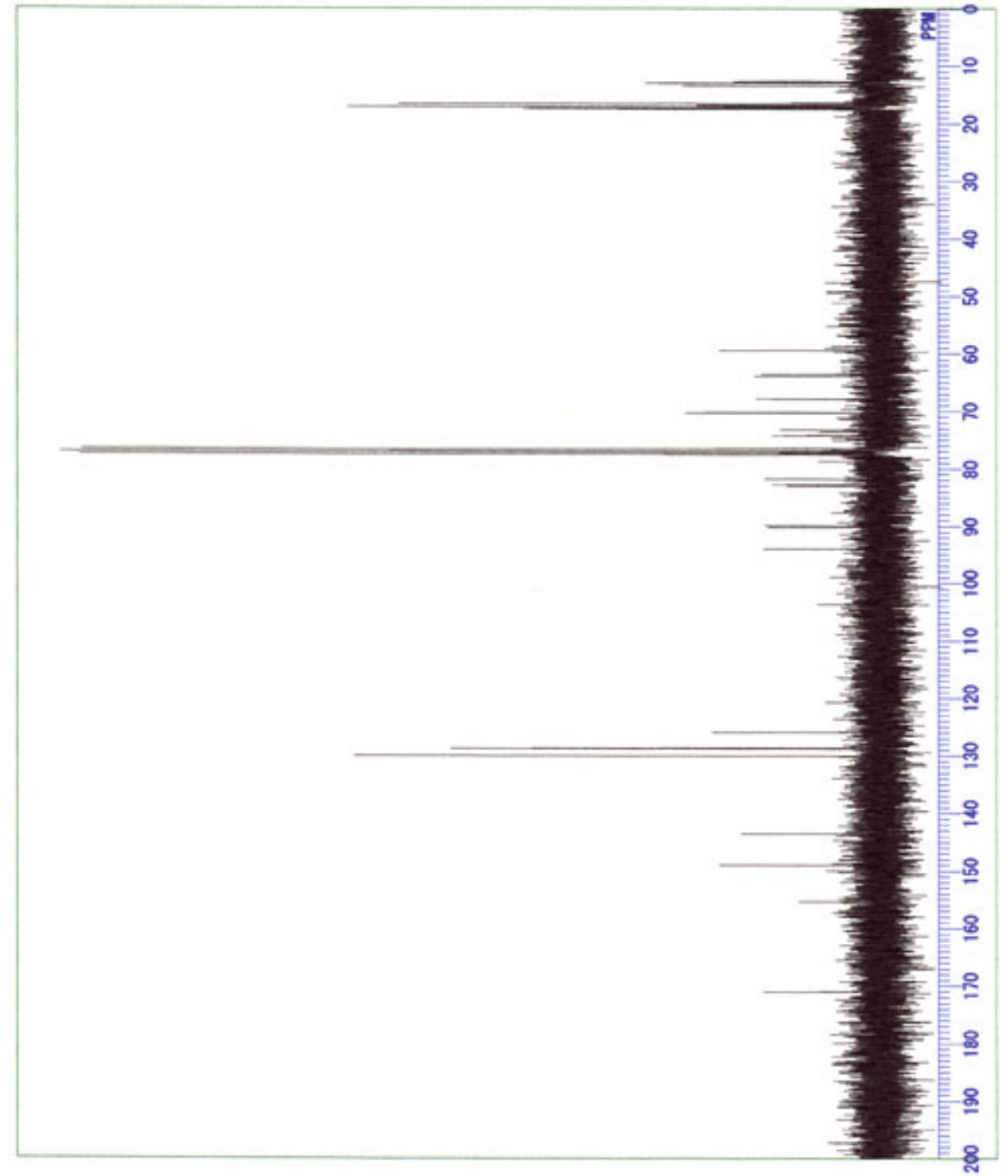




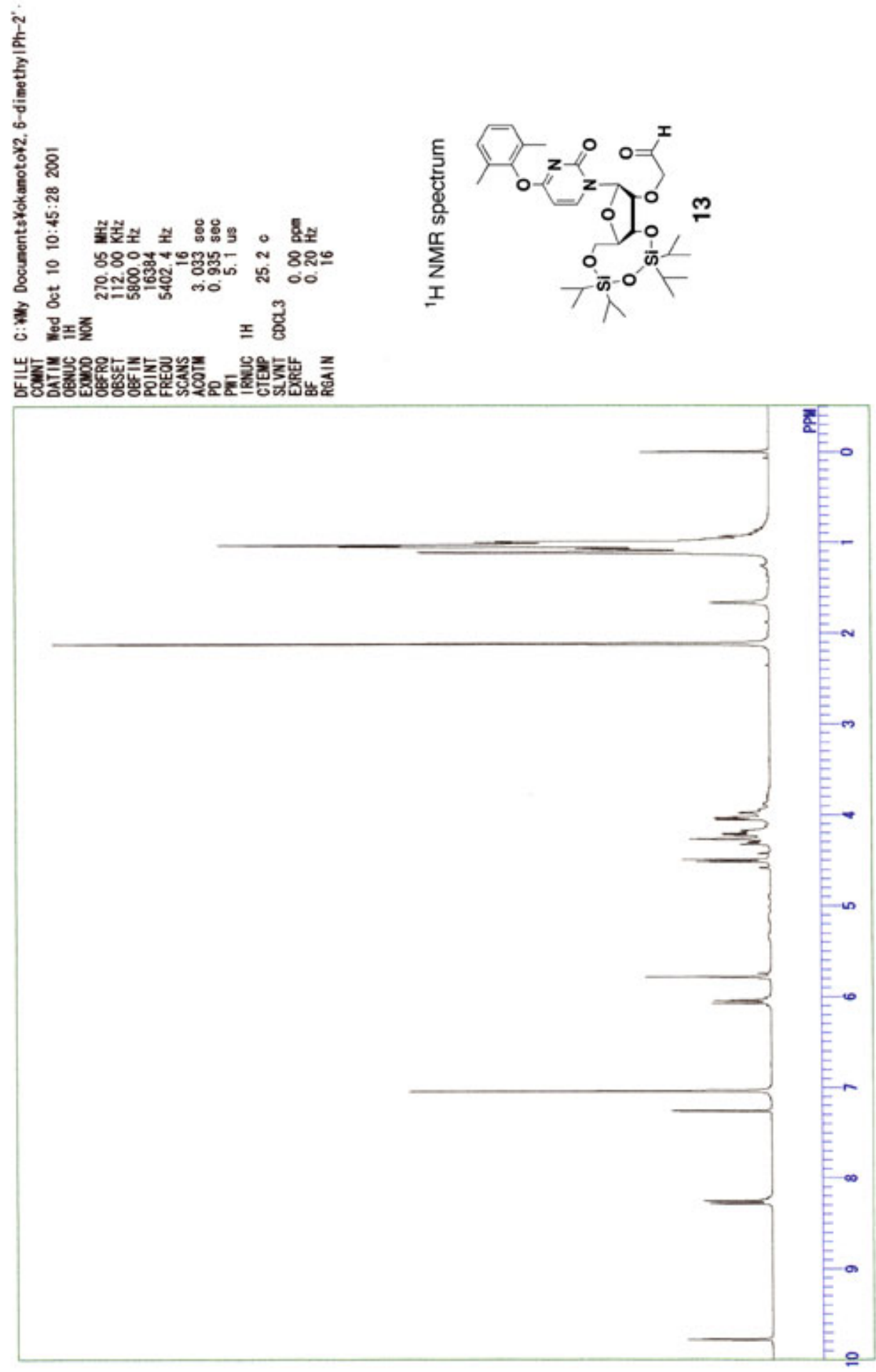




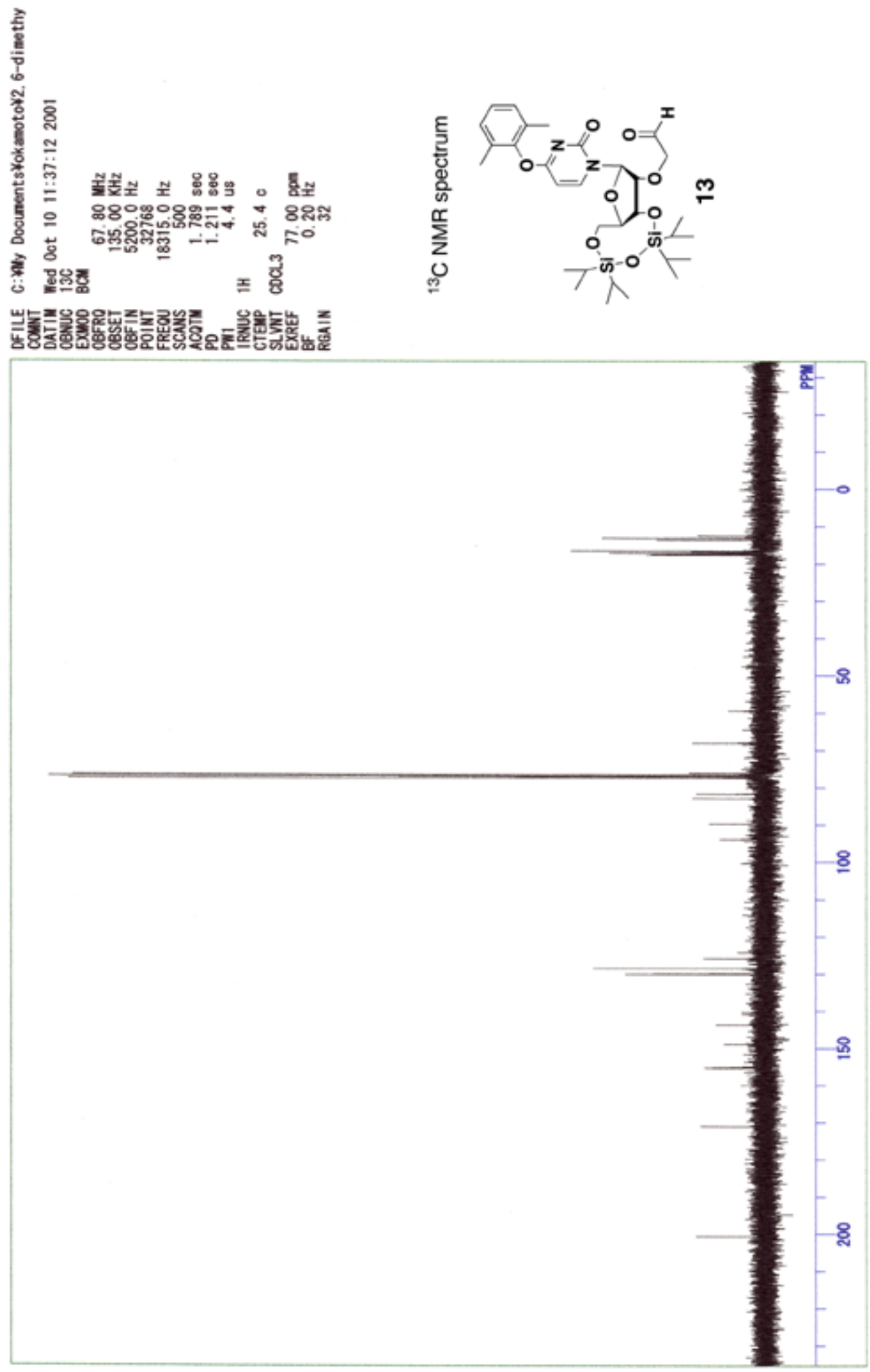




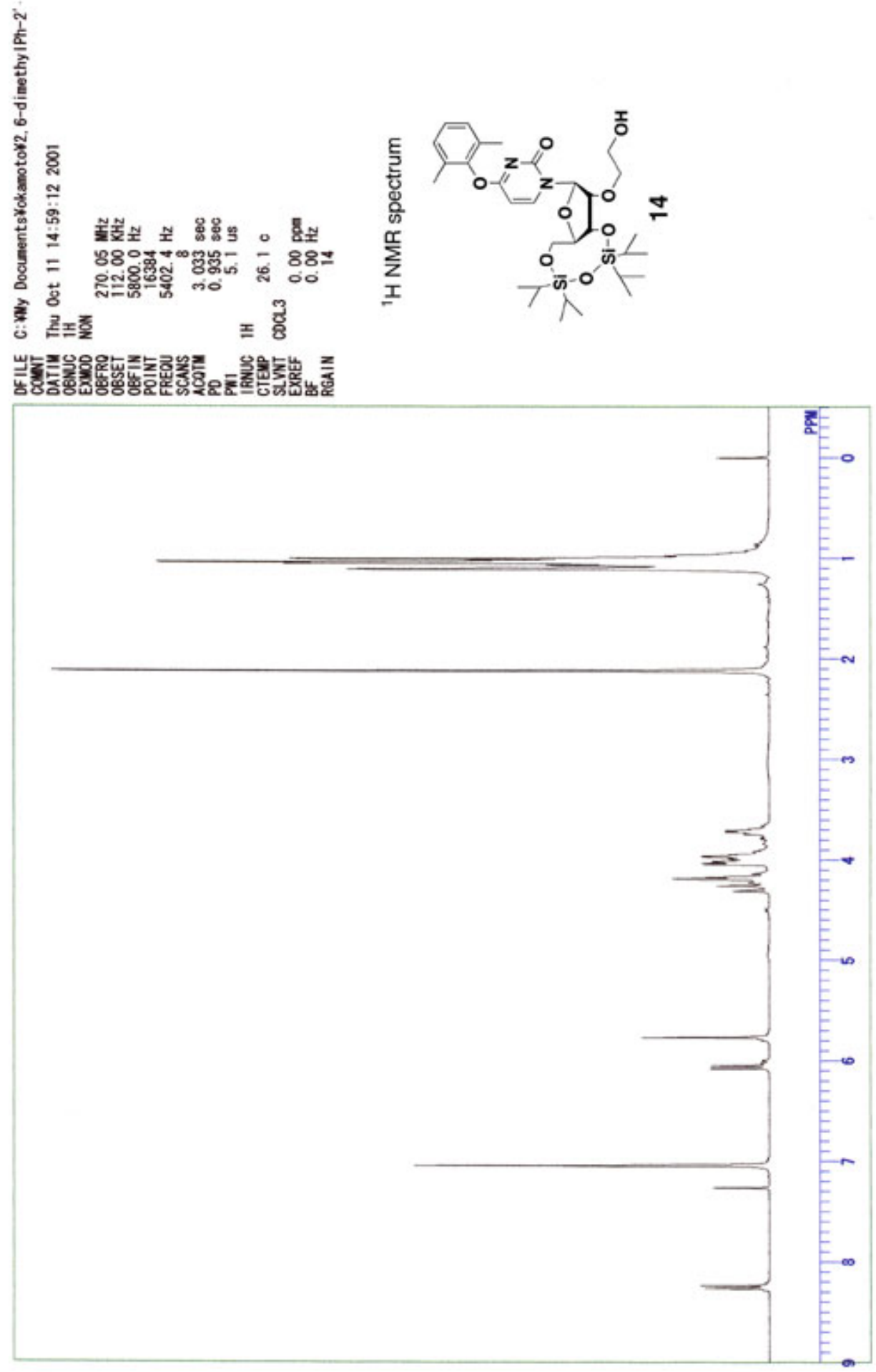

I 

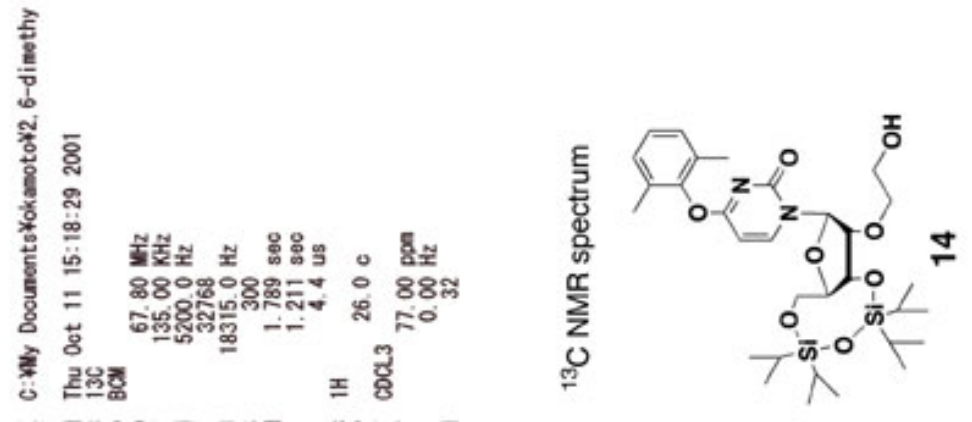

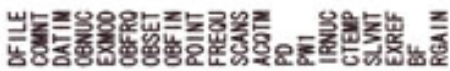

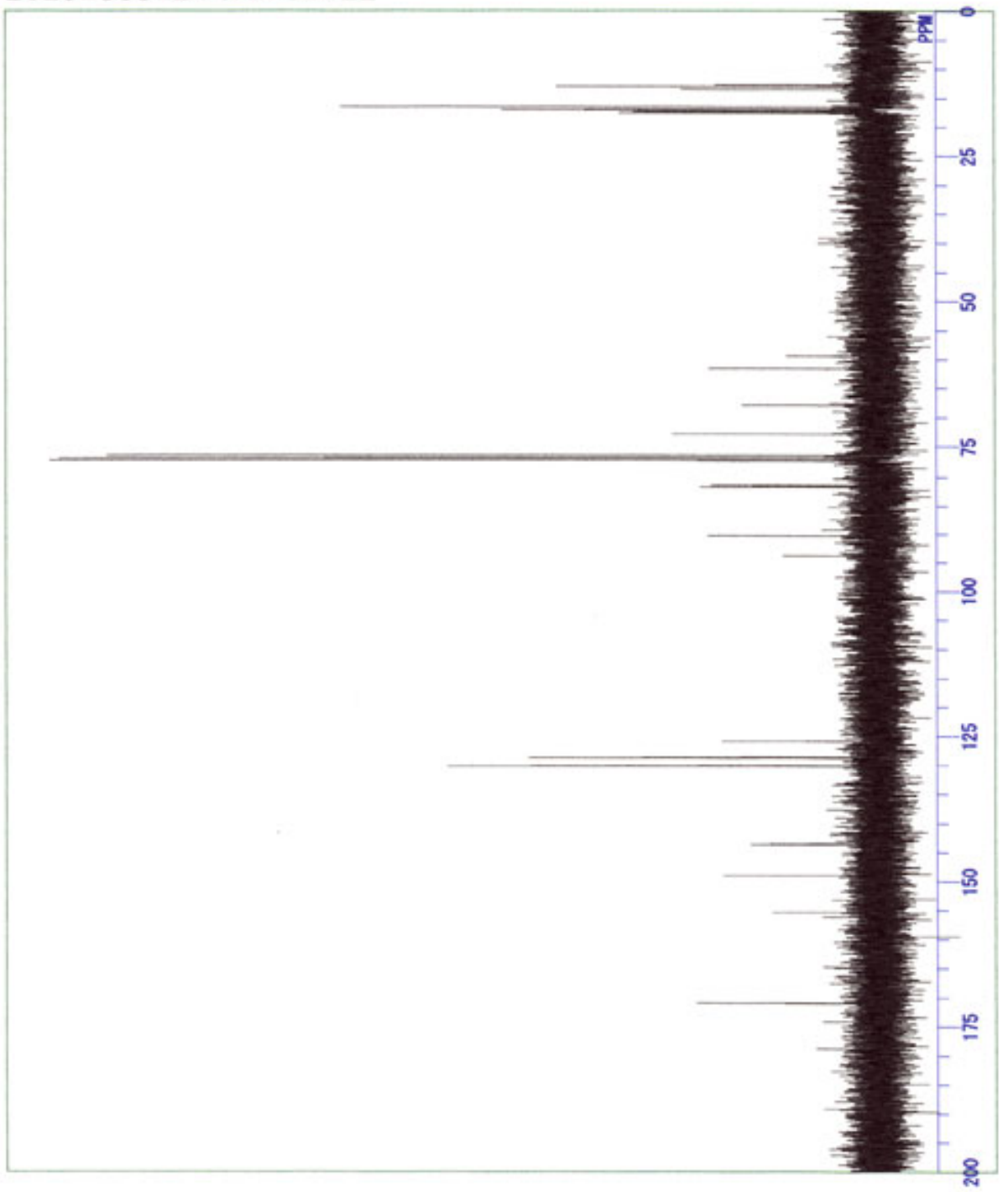




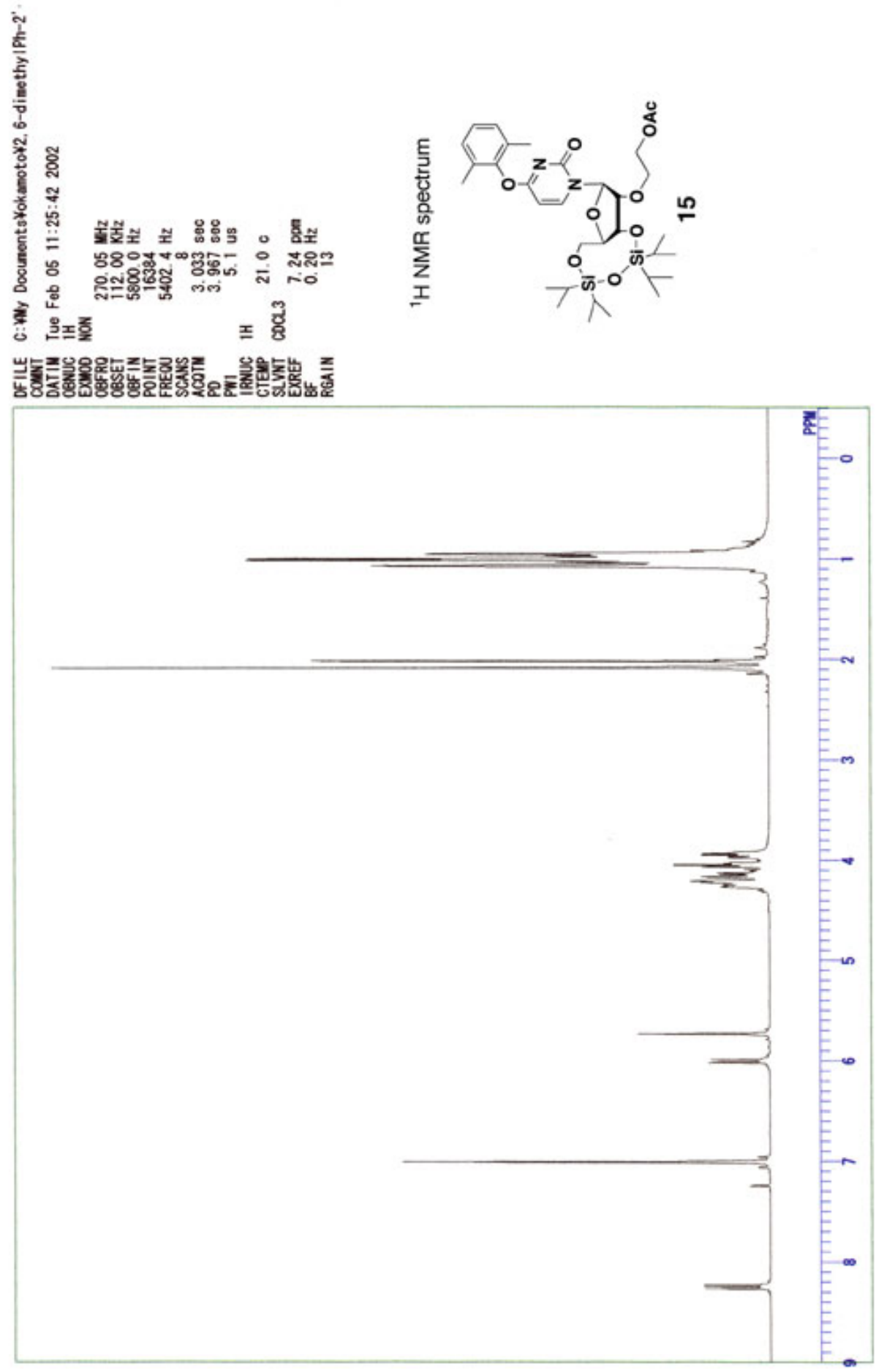




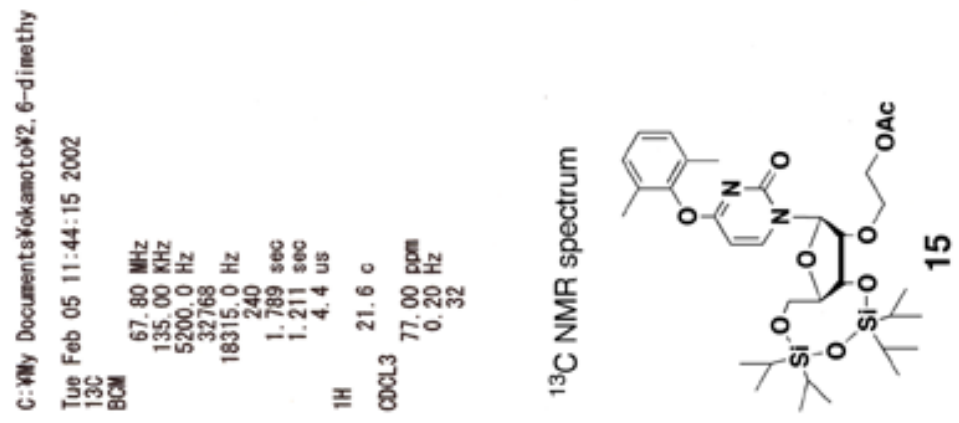

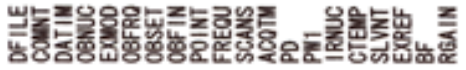

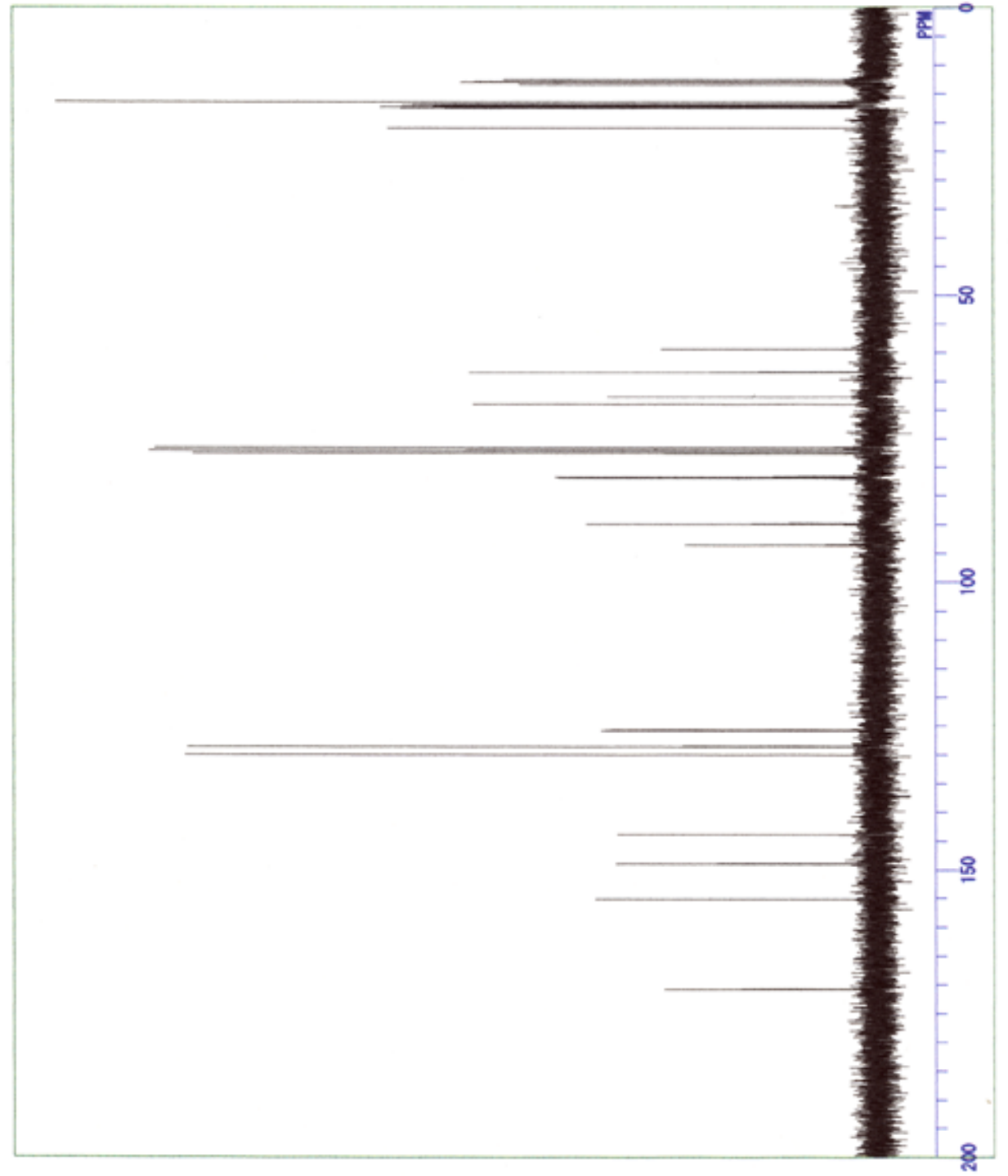




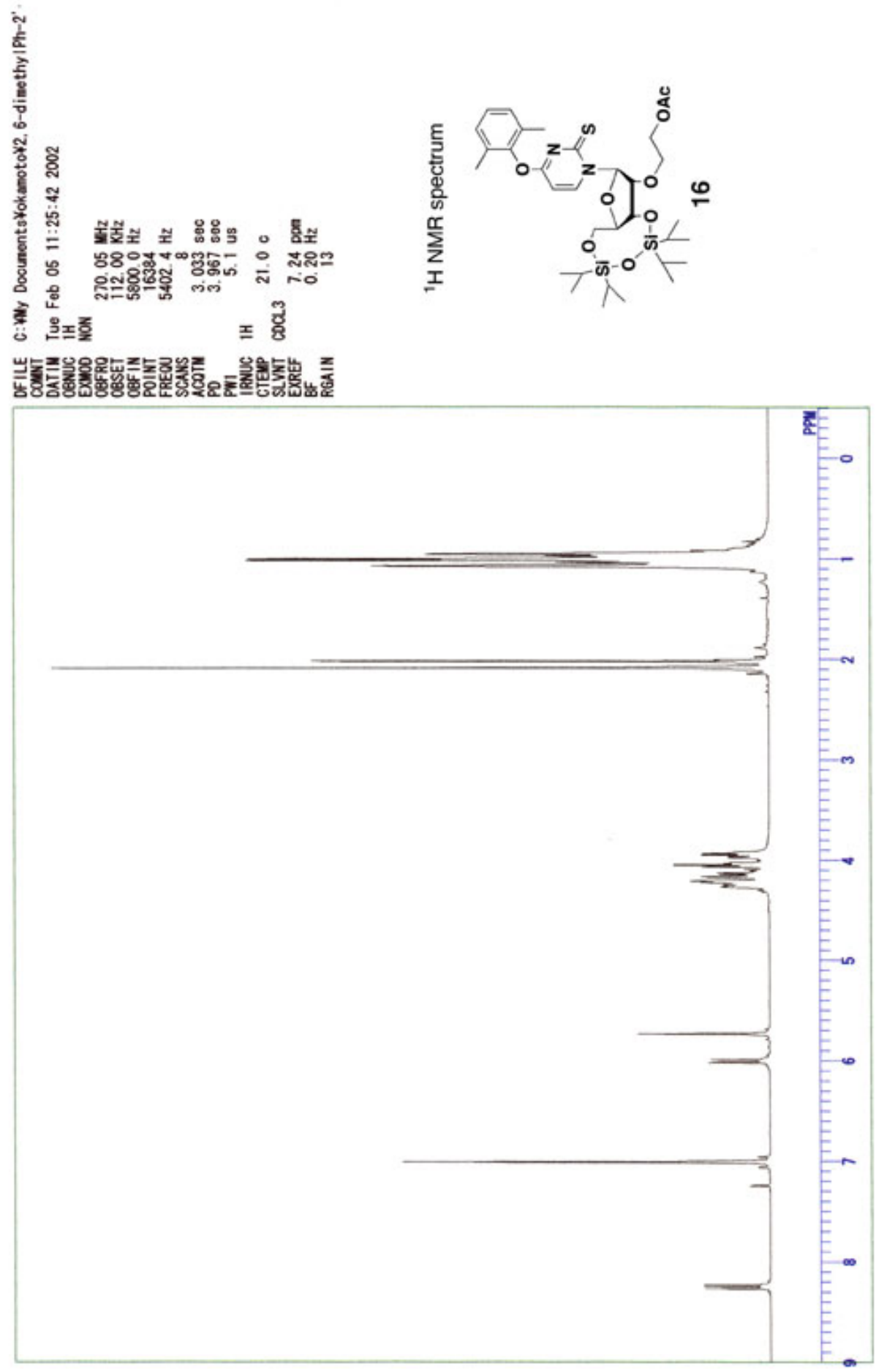




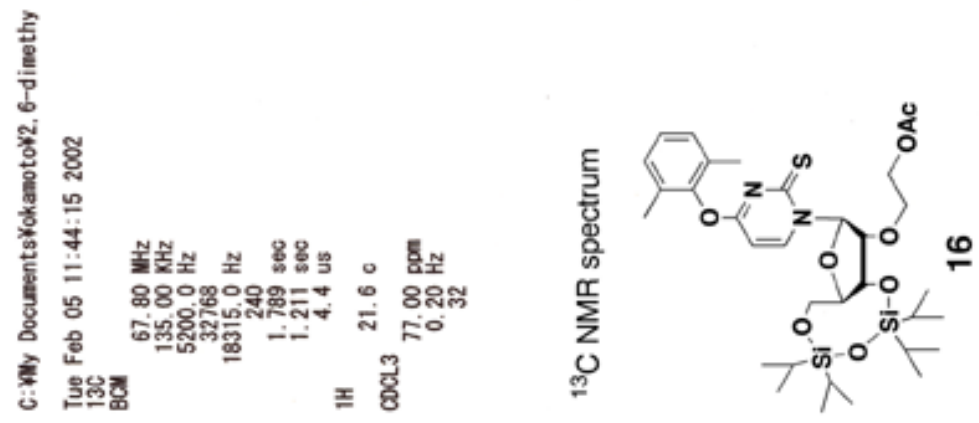

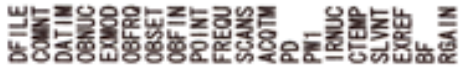

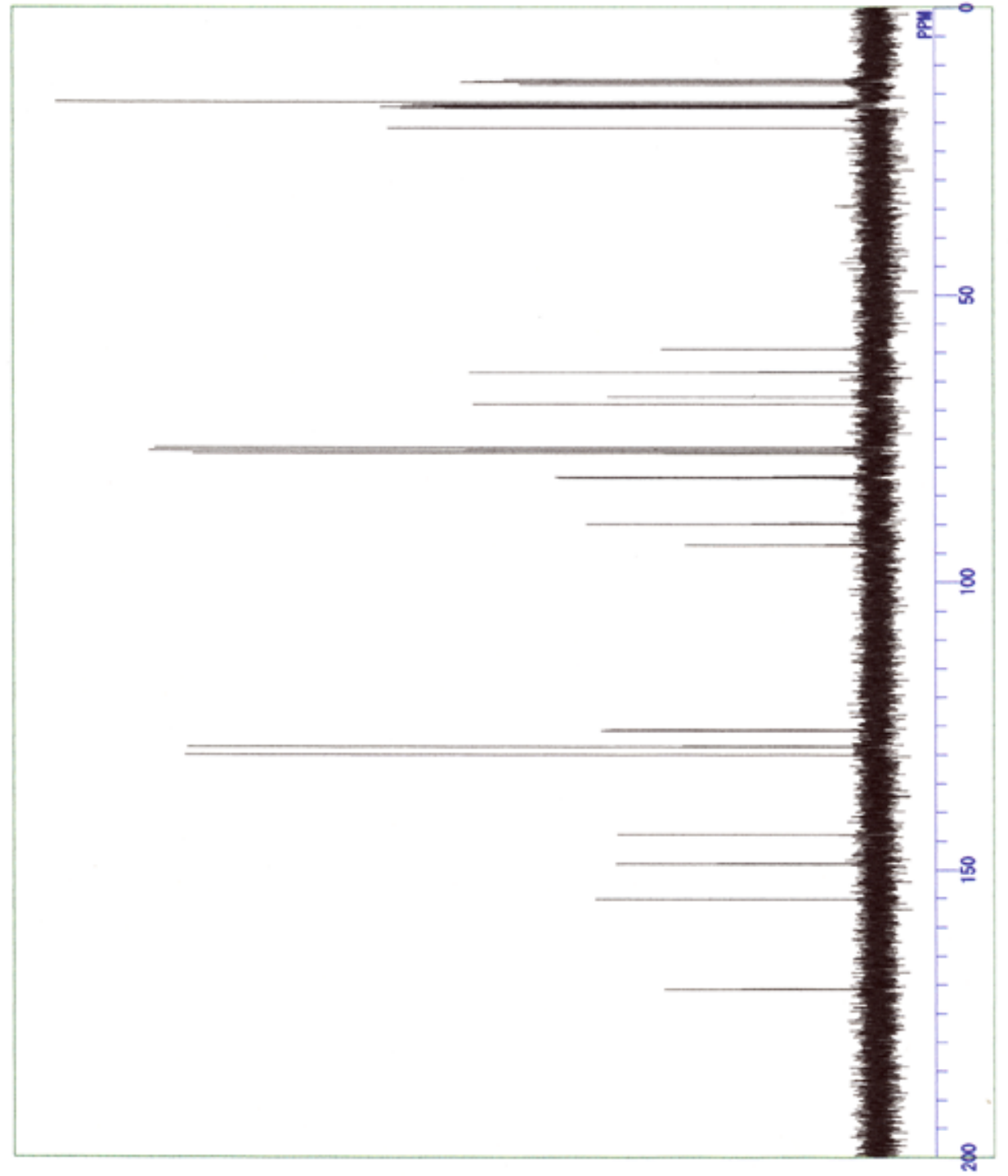




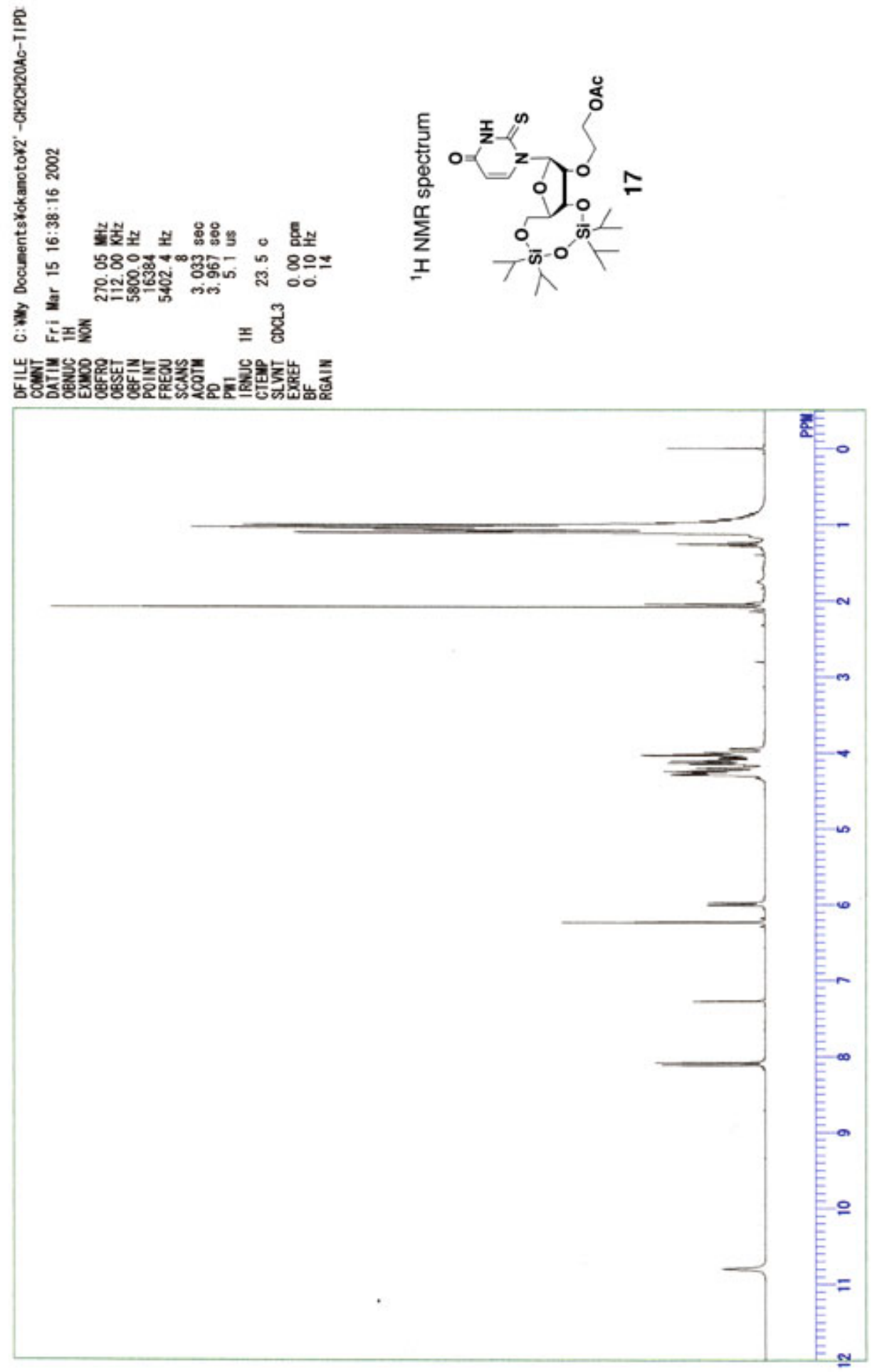

I 


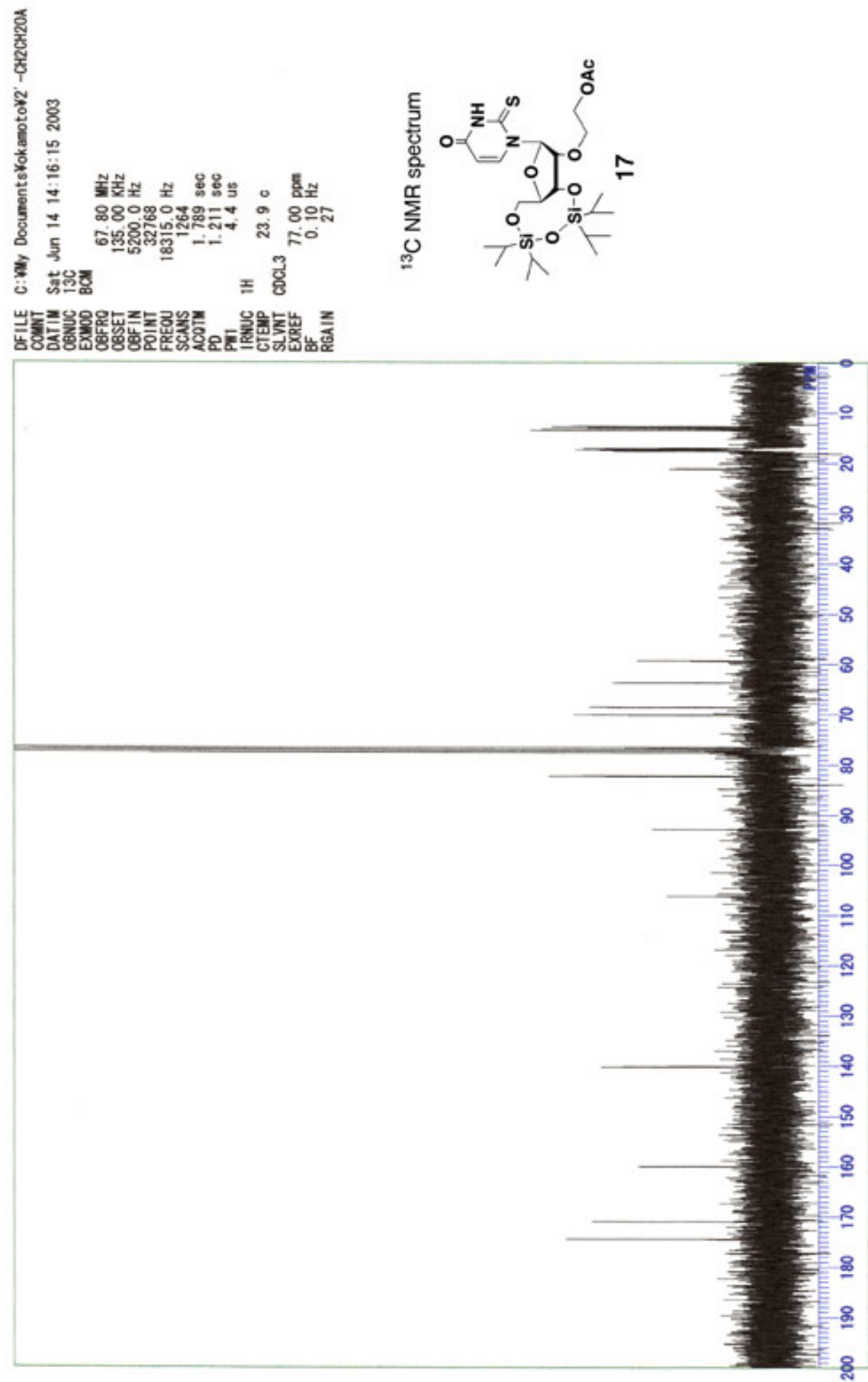




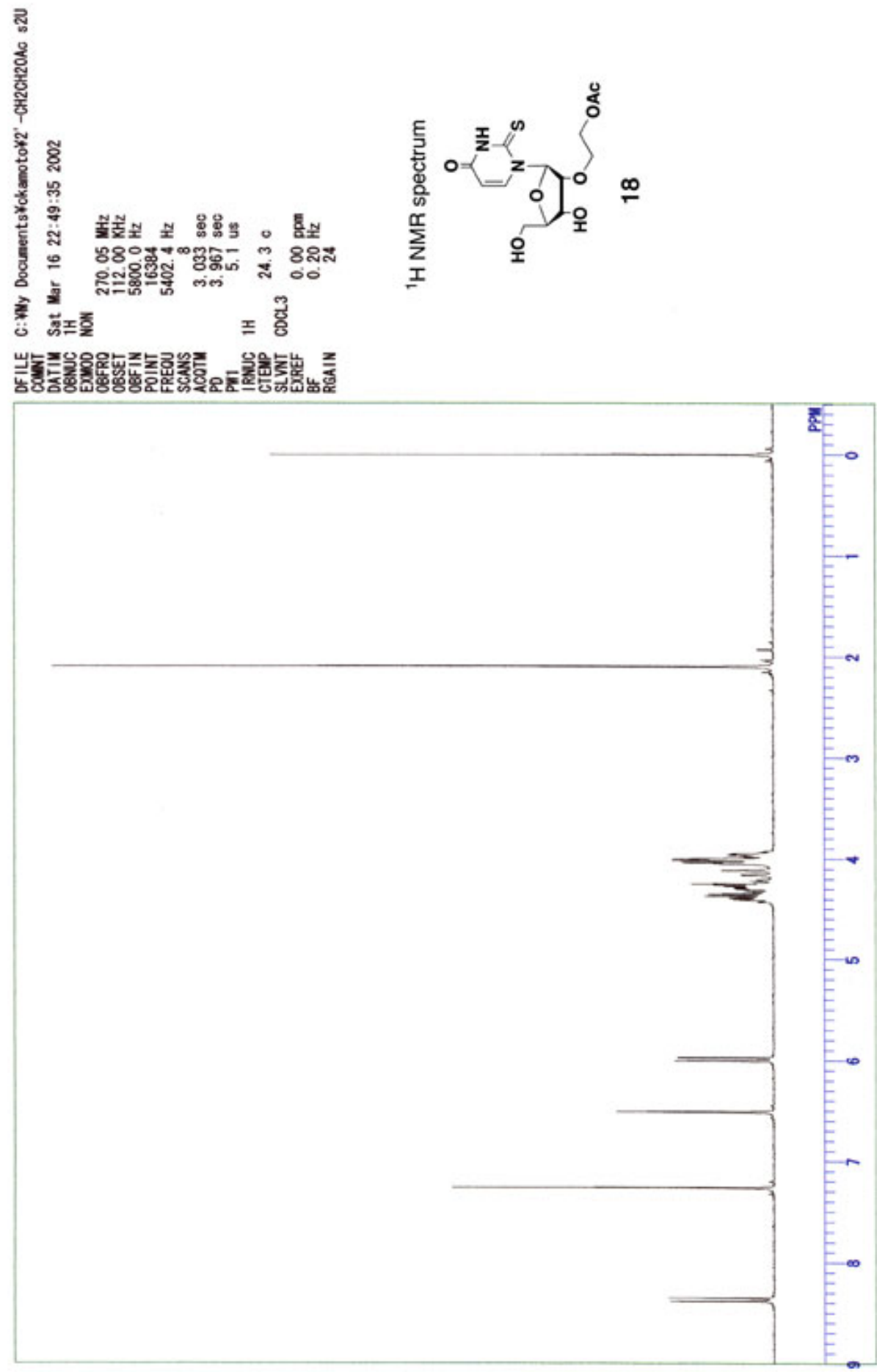




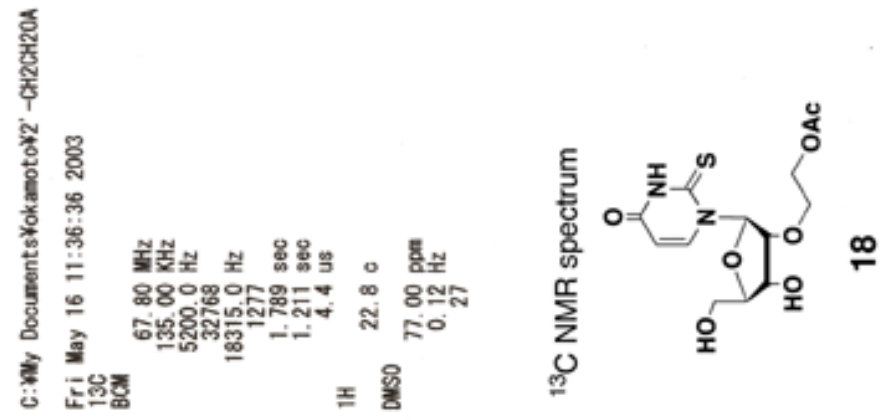

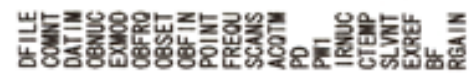

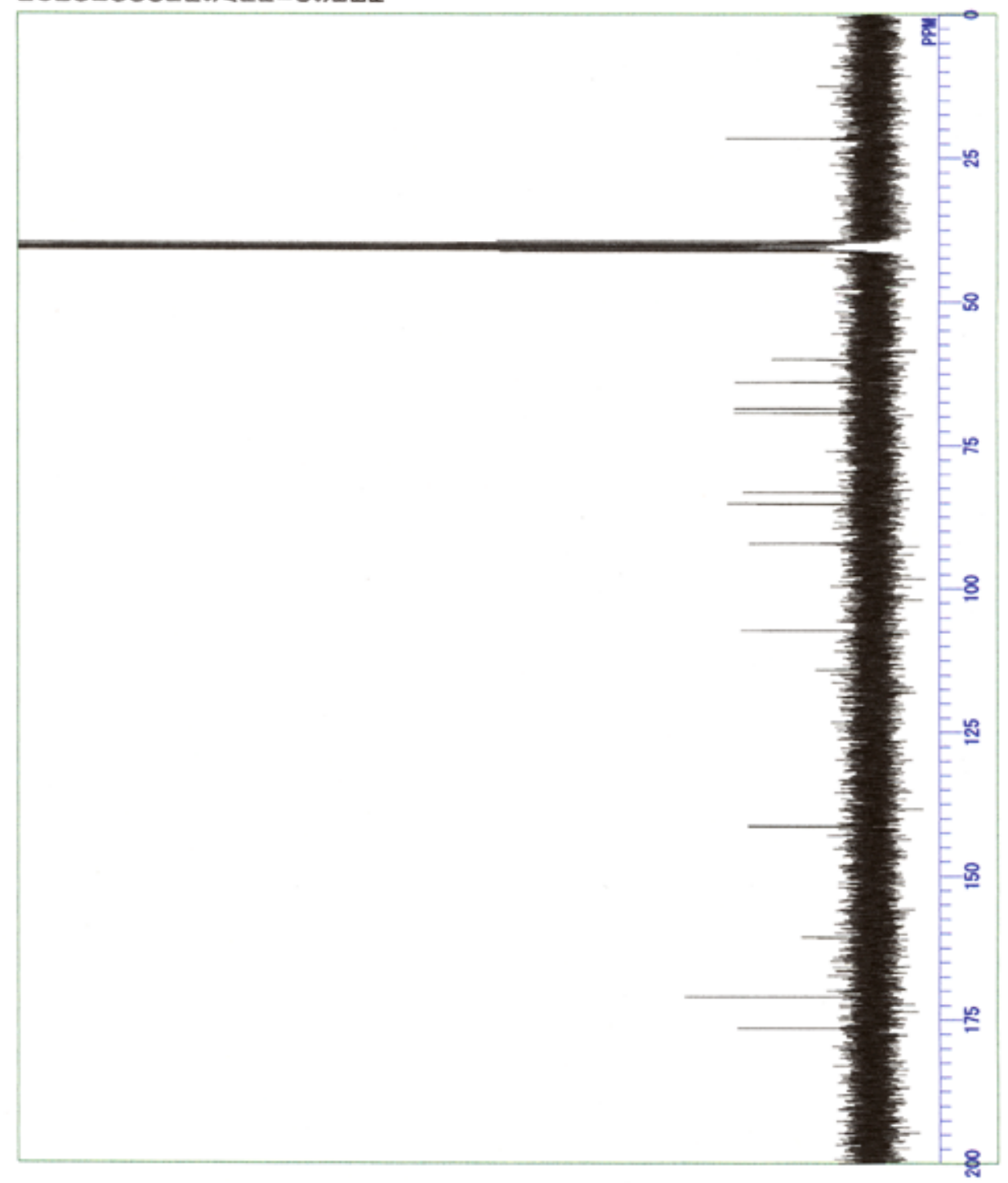




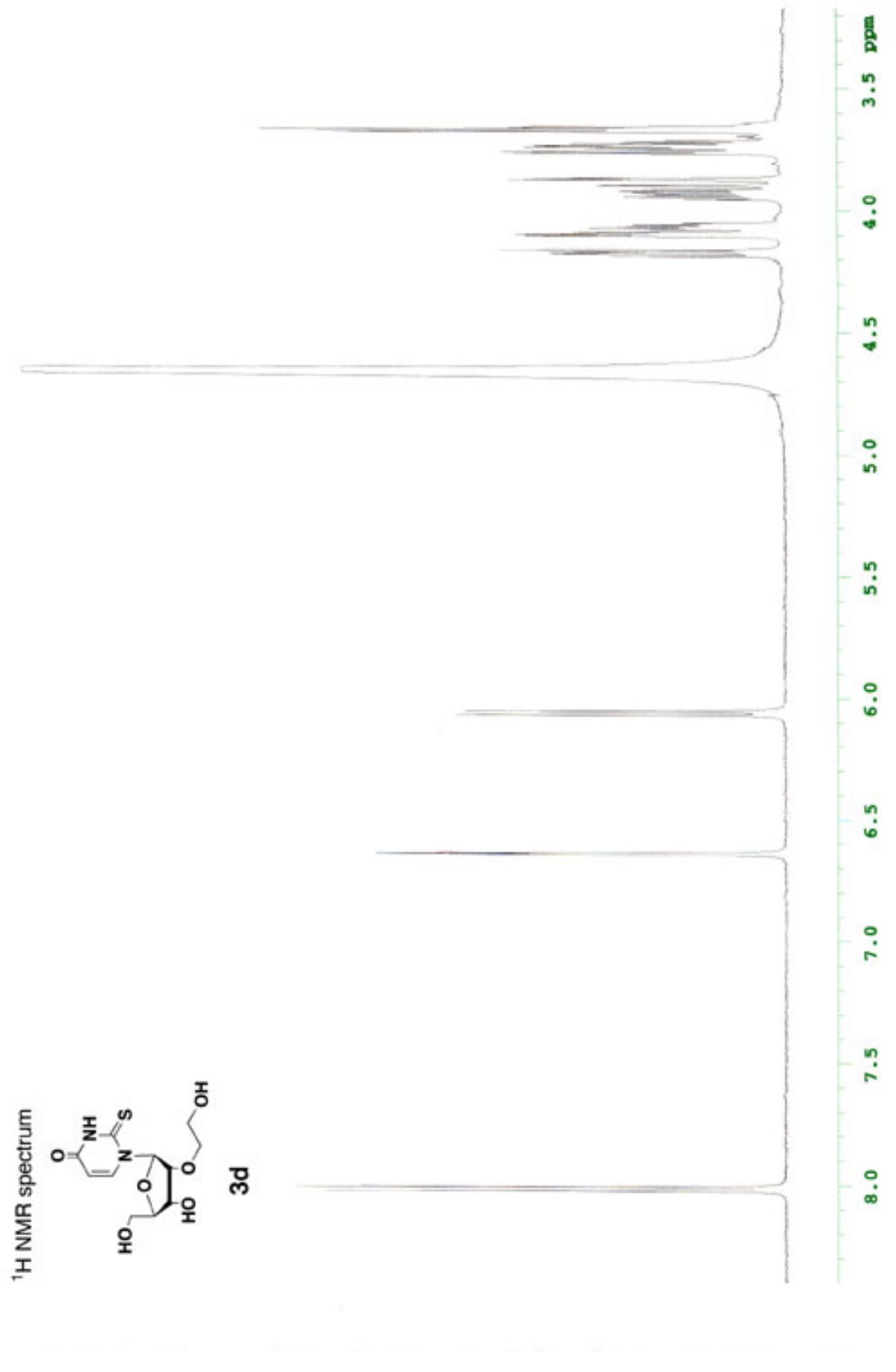




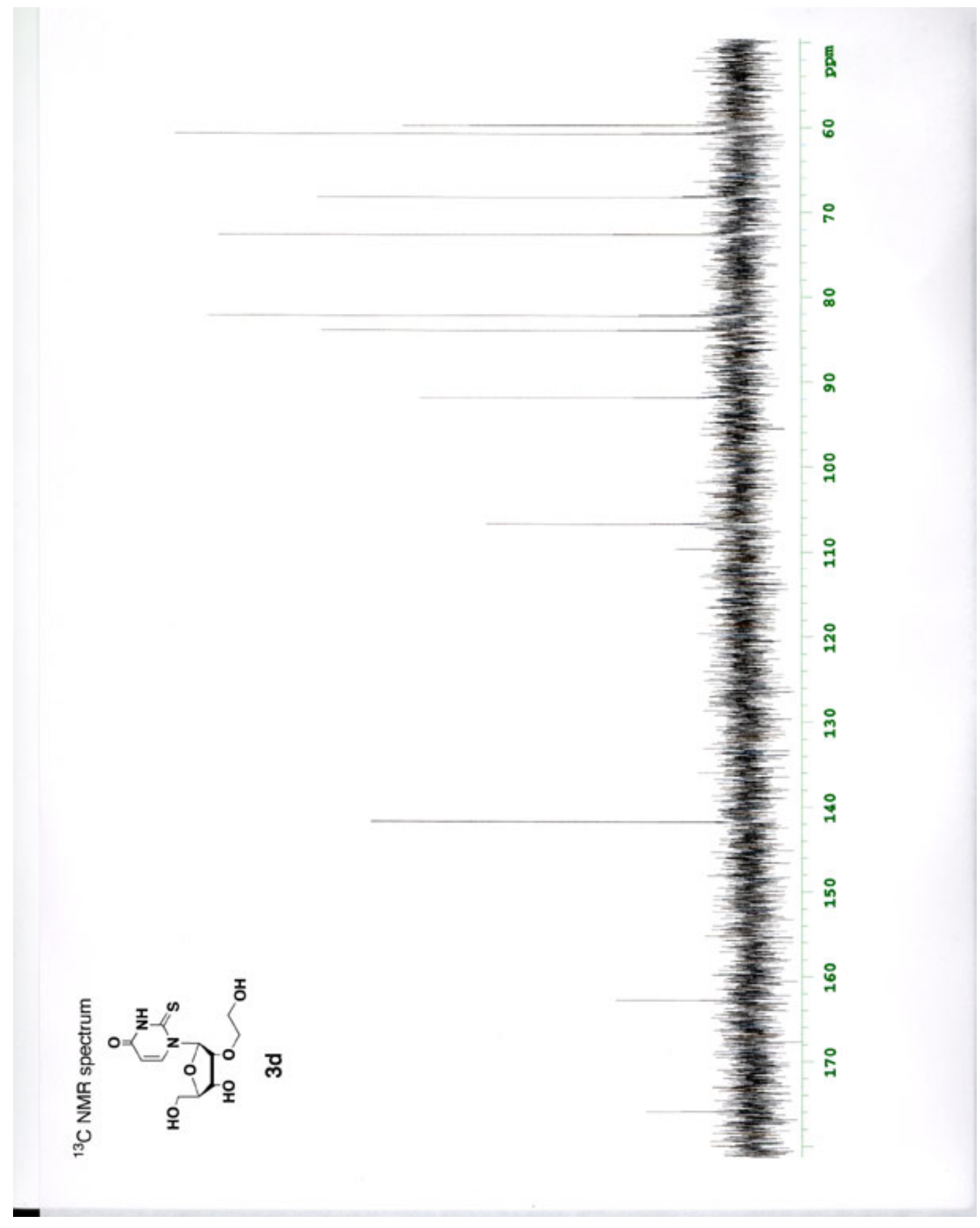




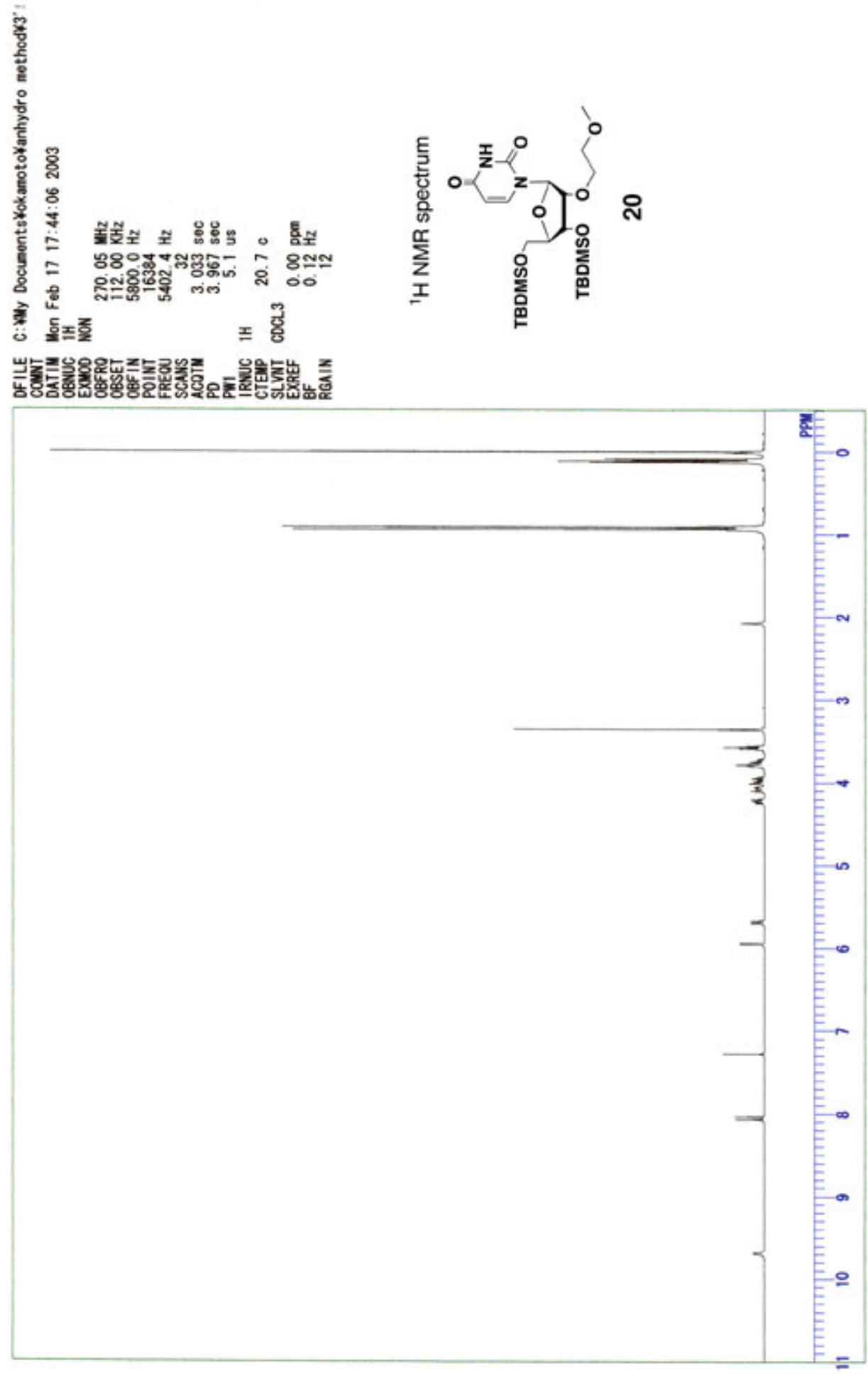




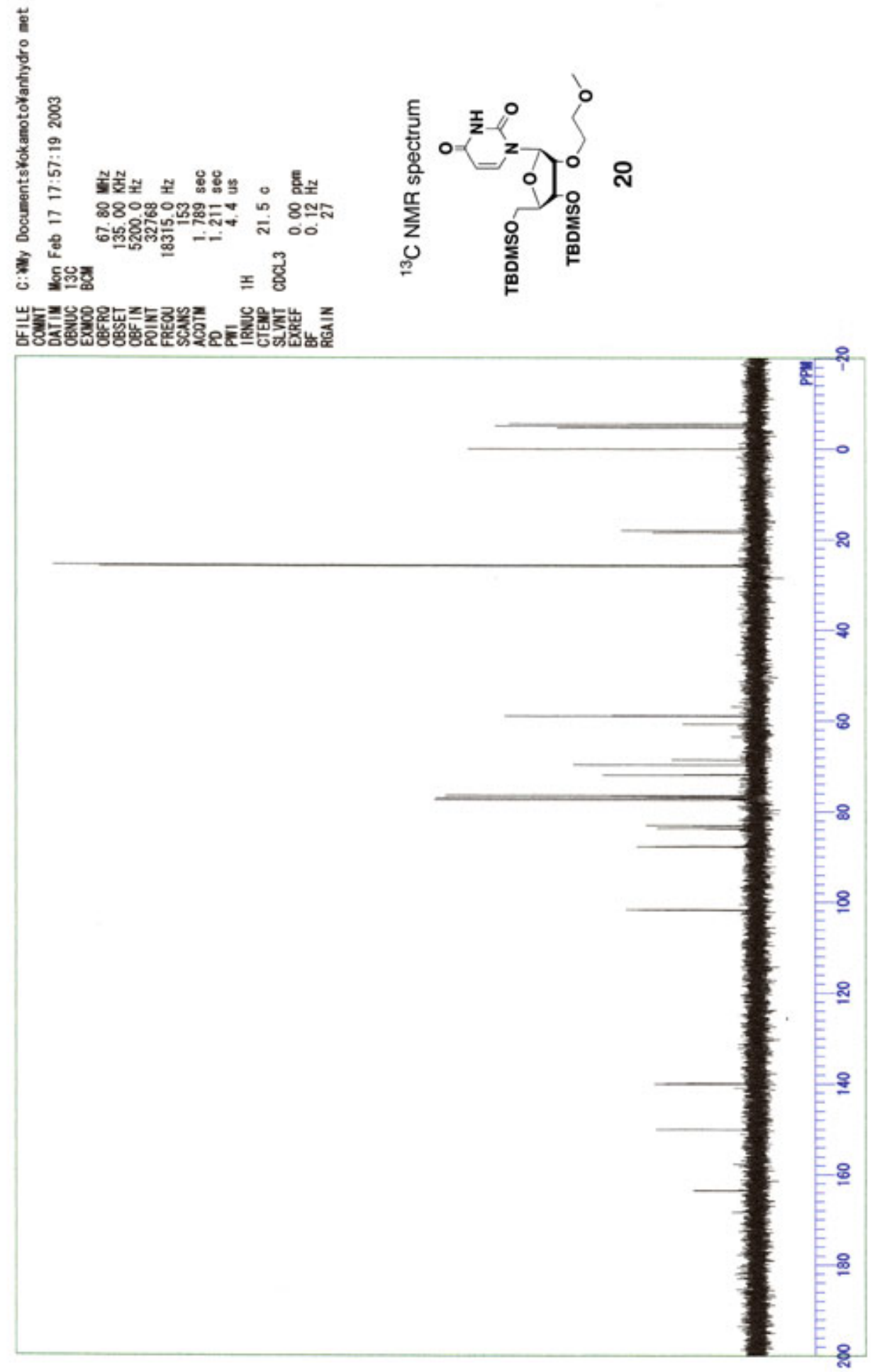




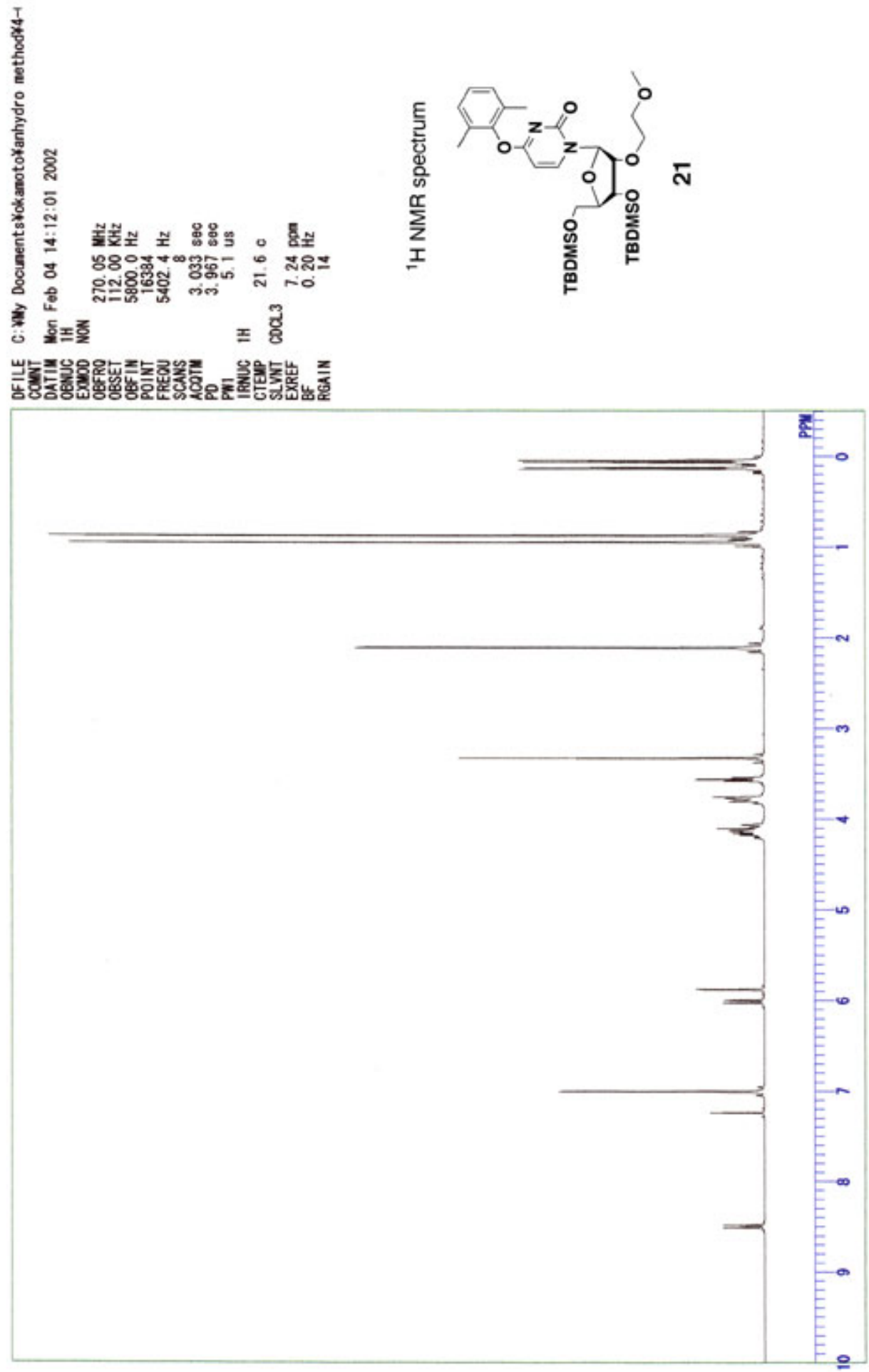

I 


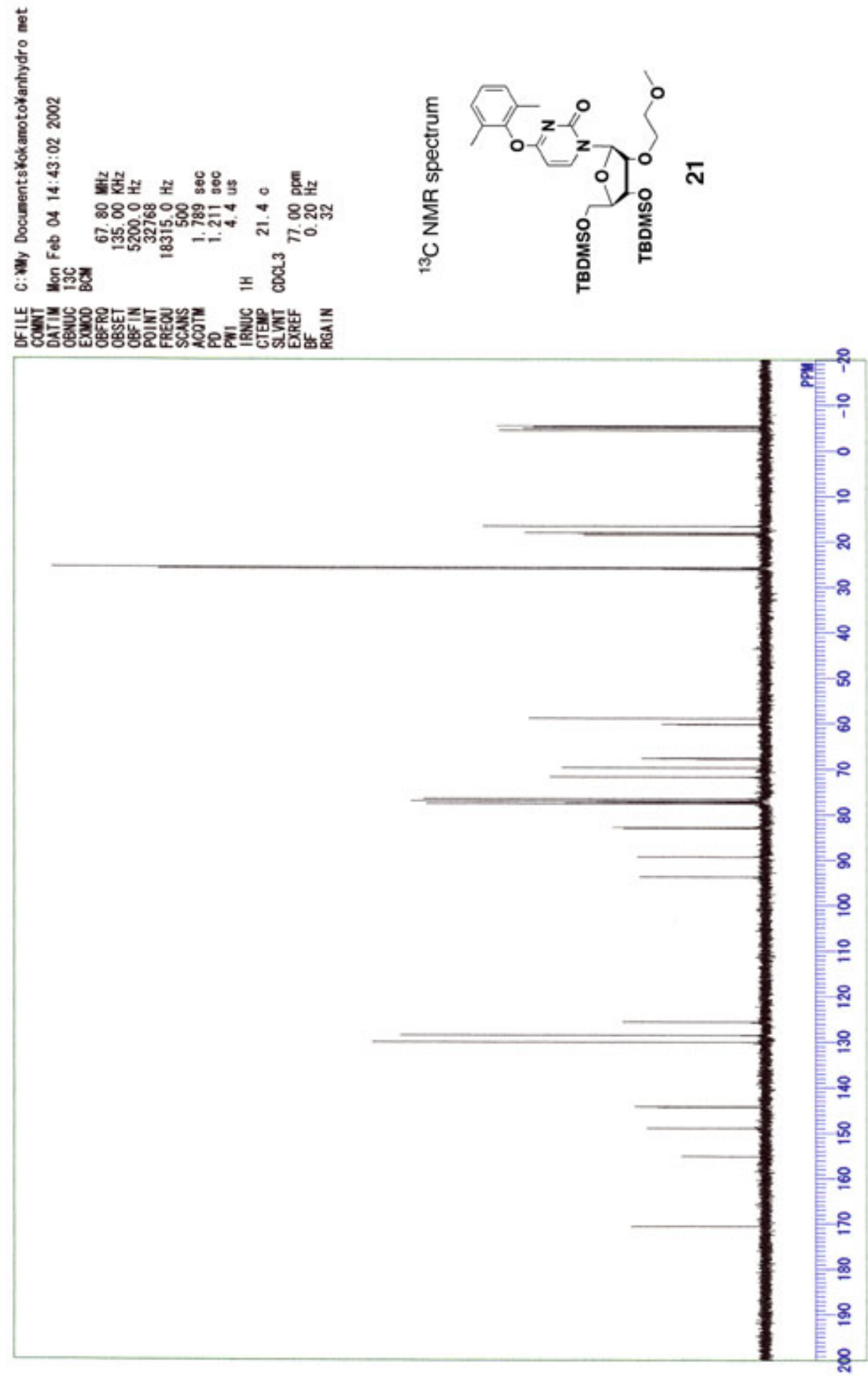

I 


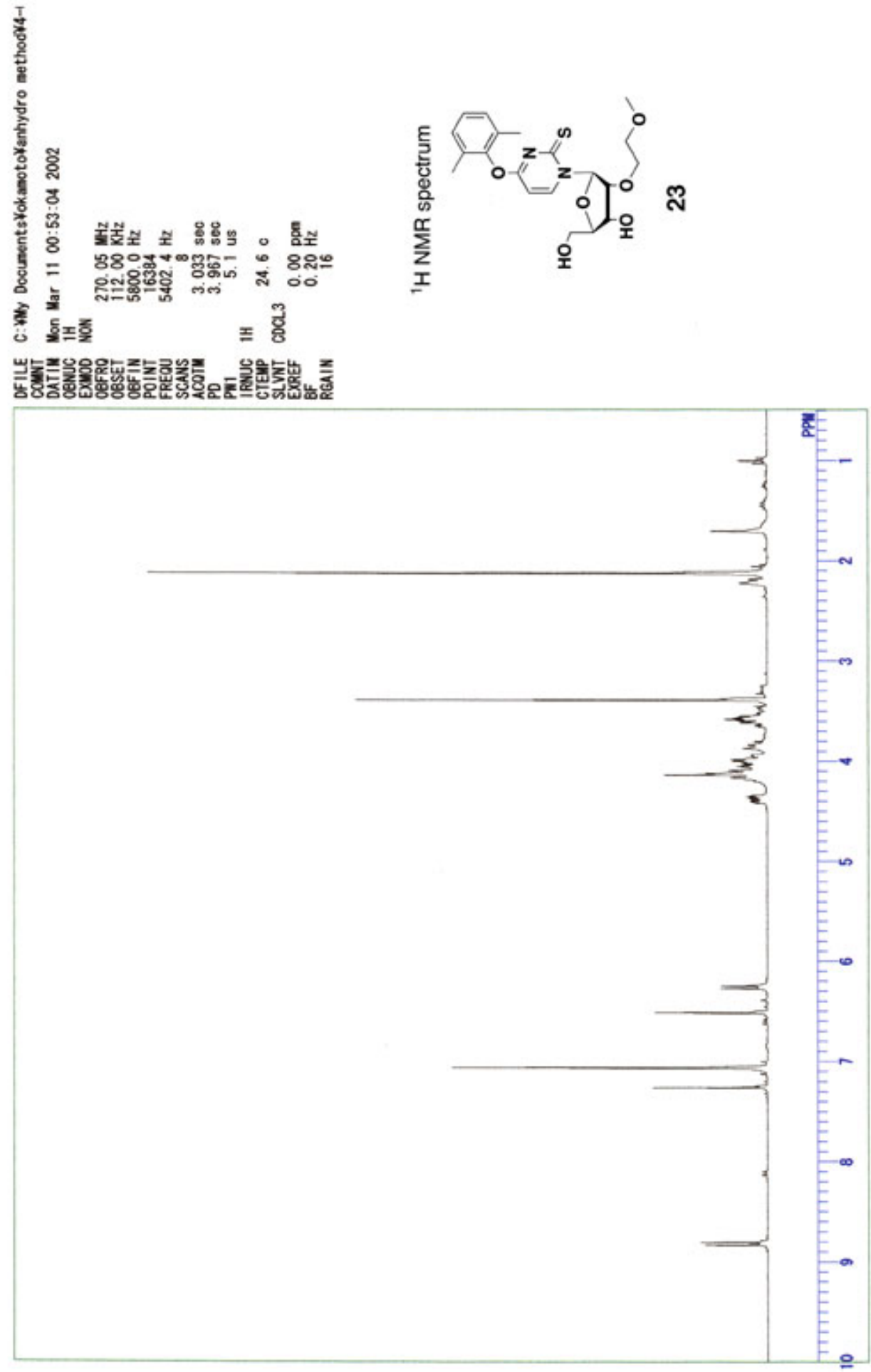




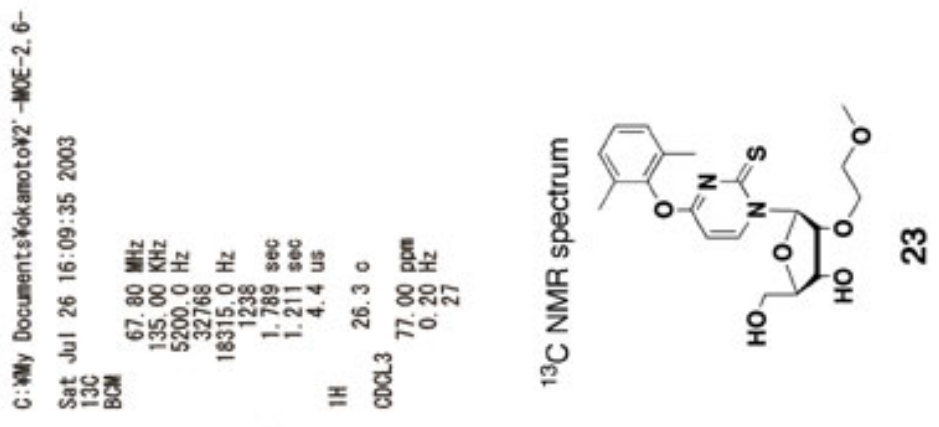

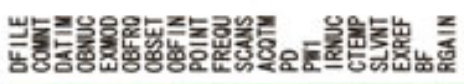

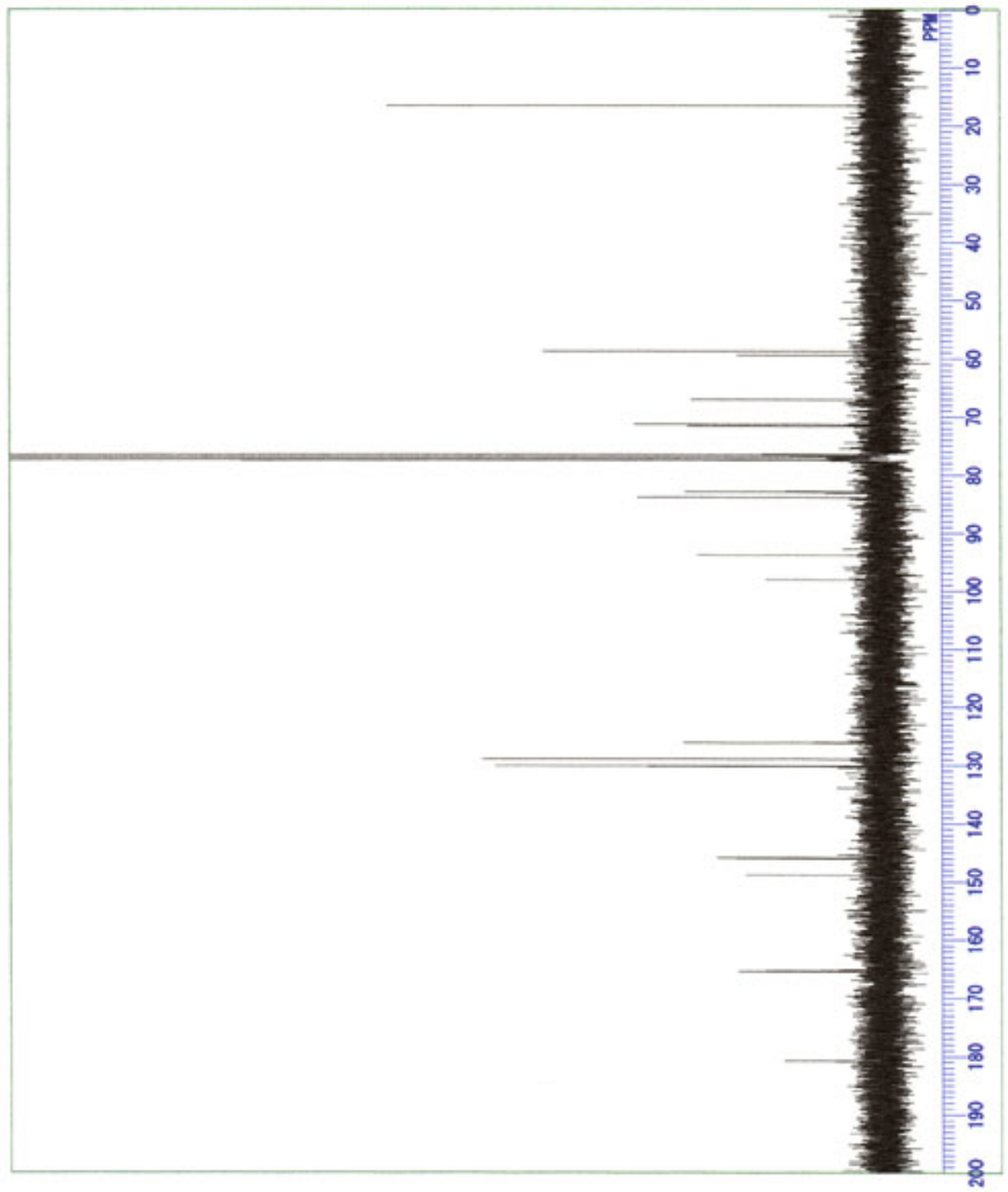




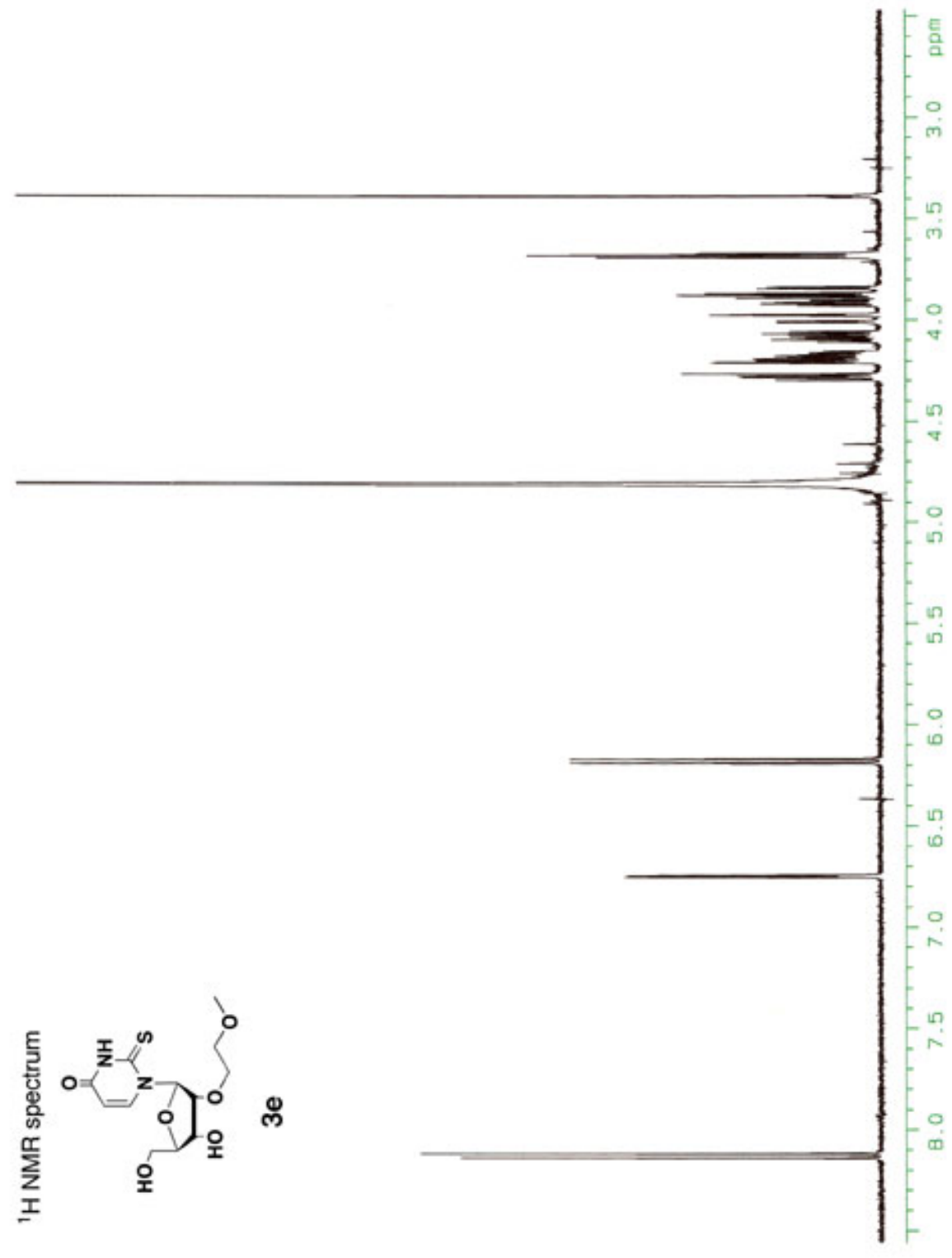




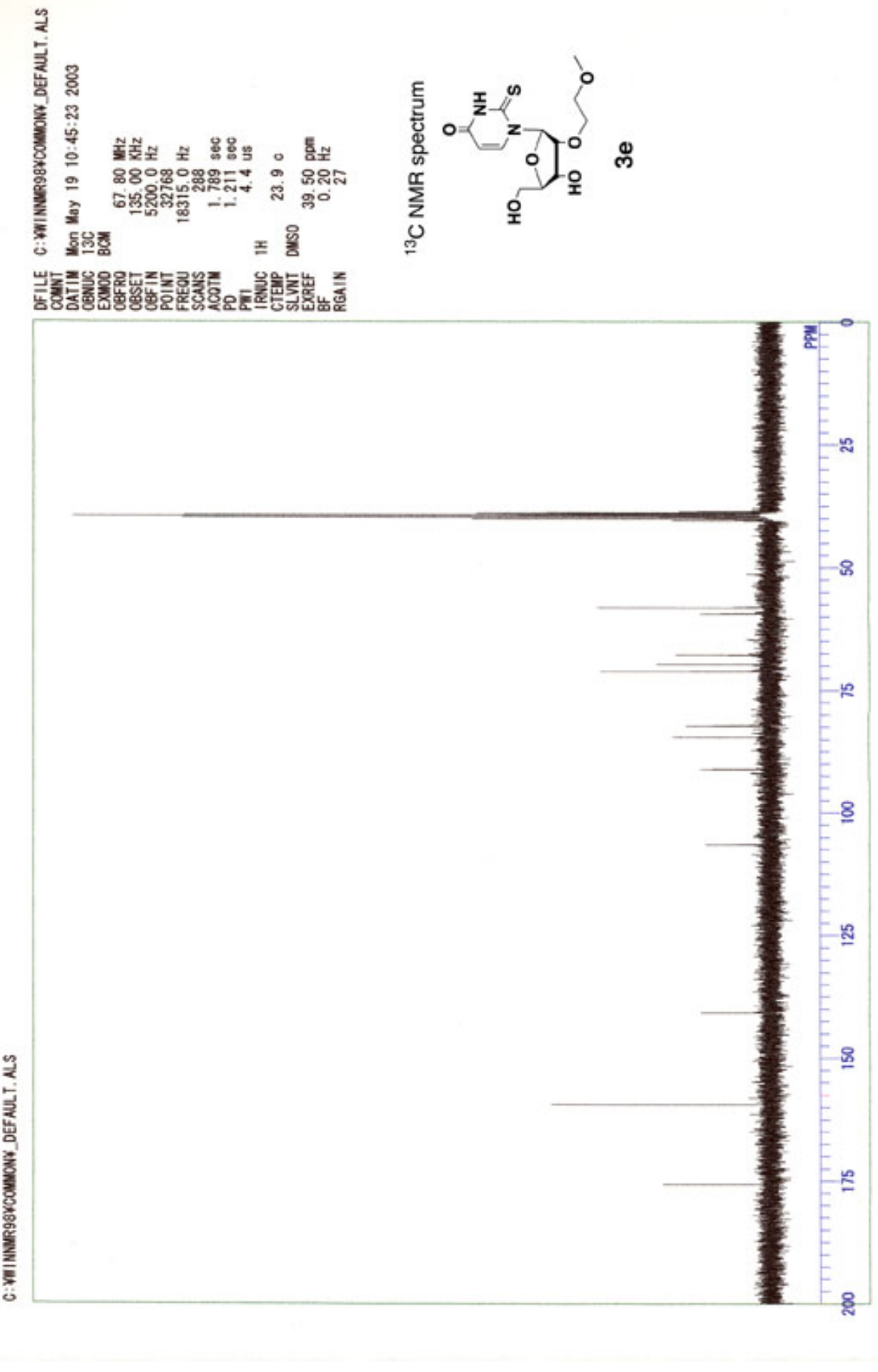



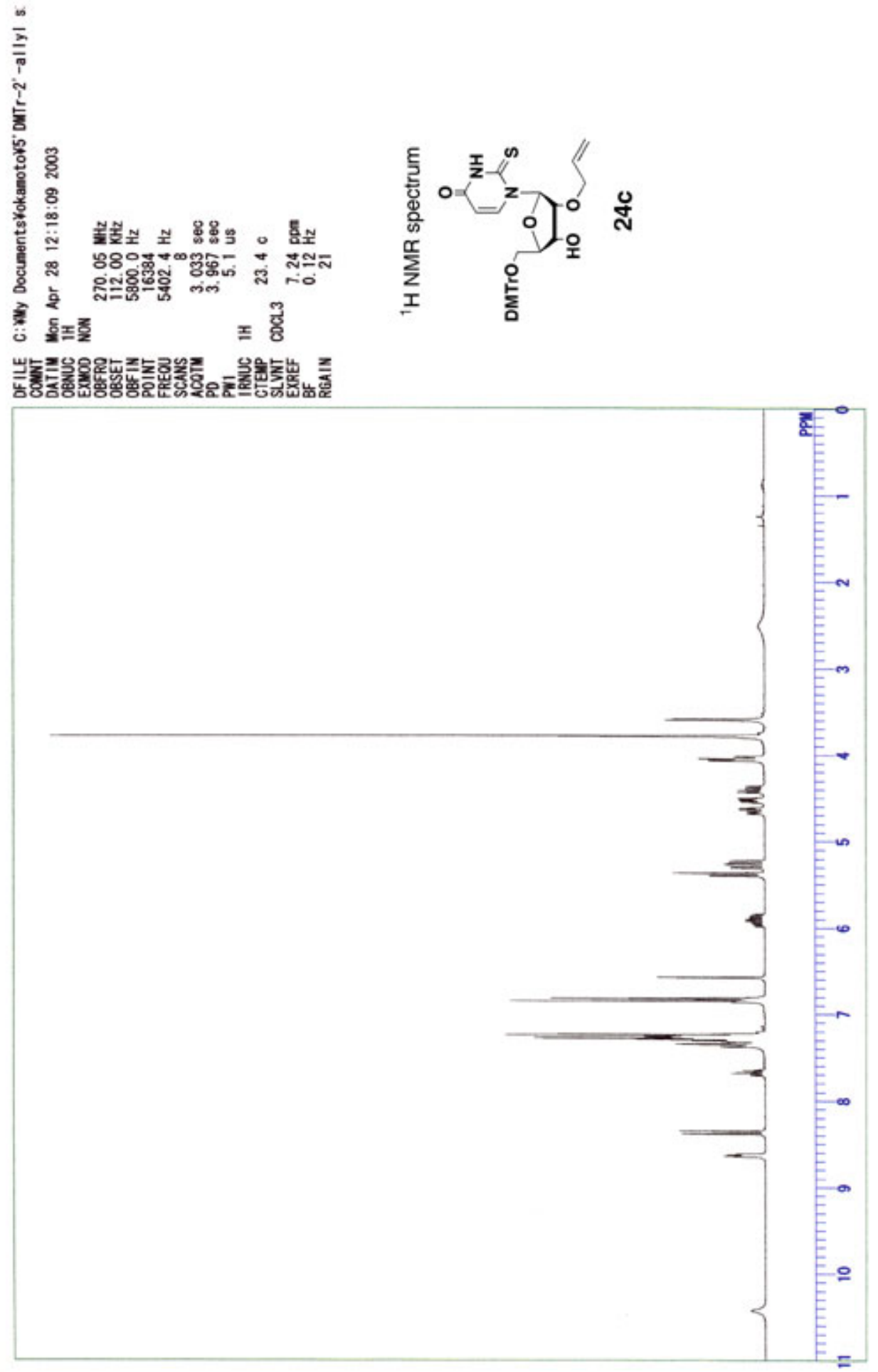


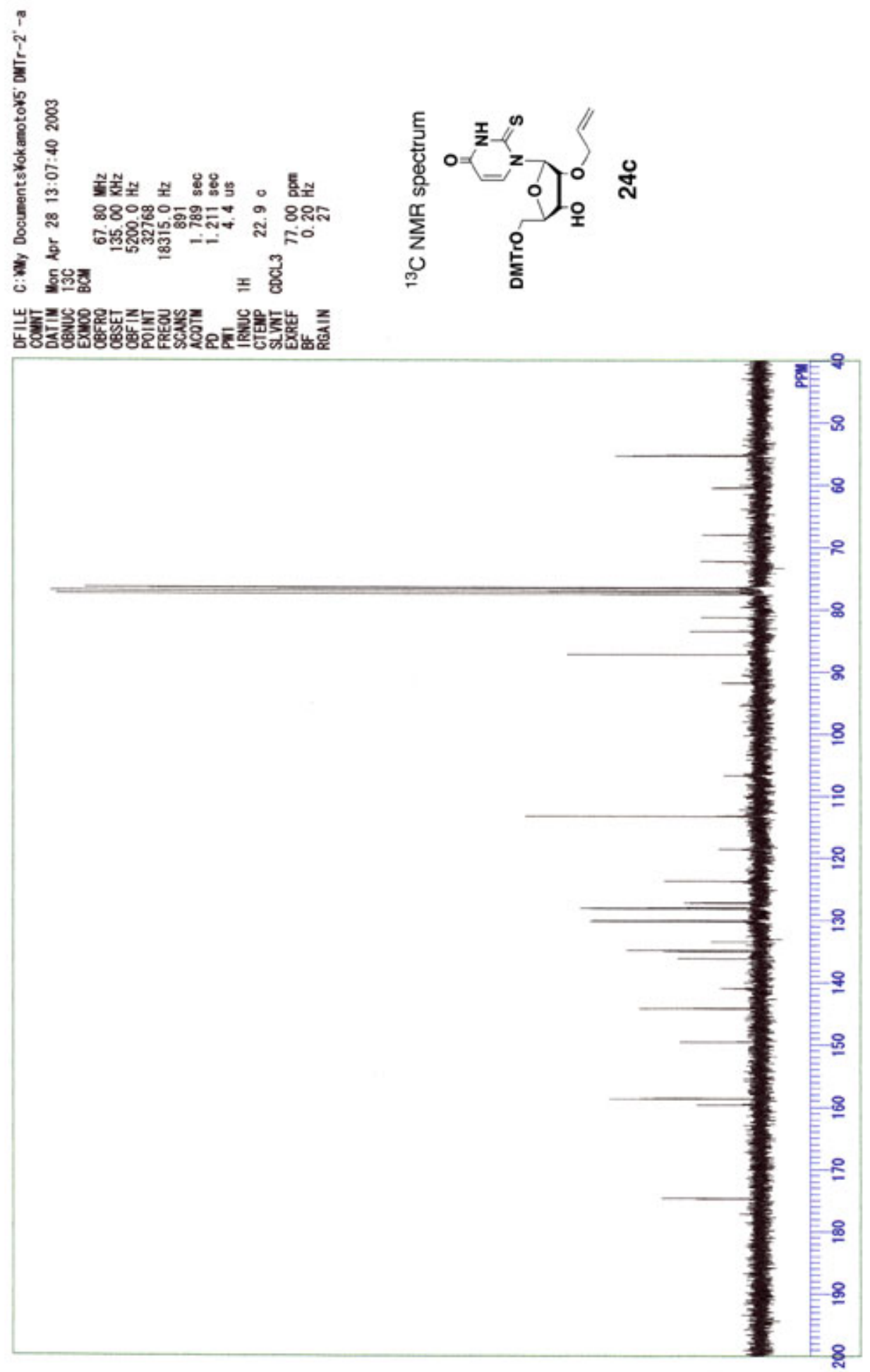

I 


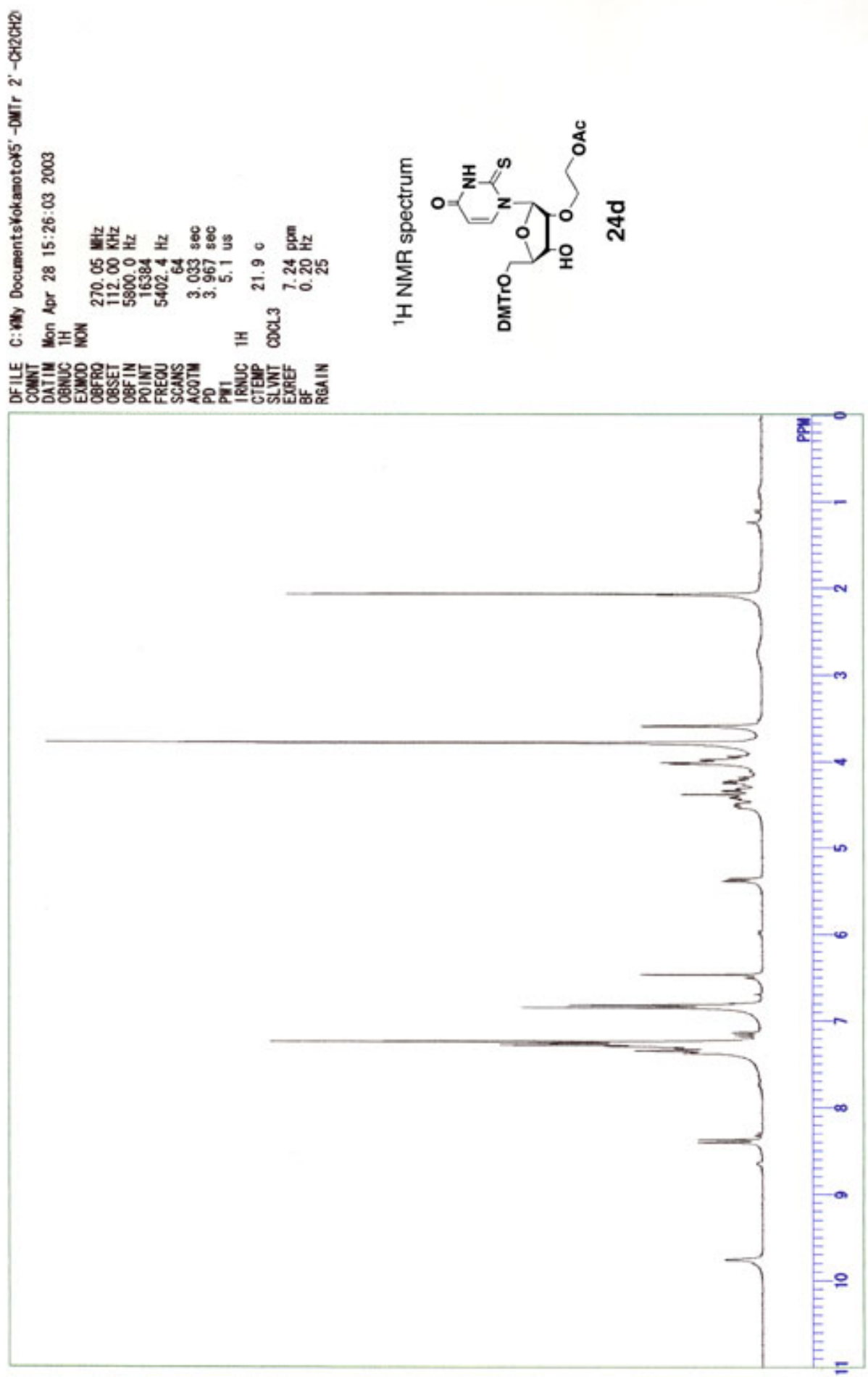




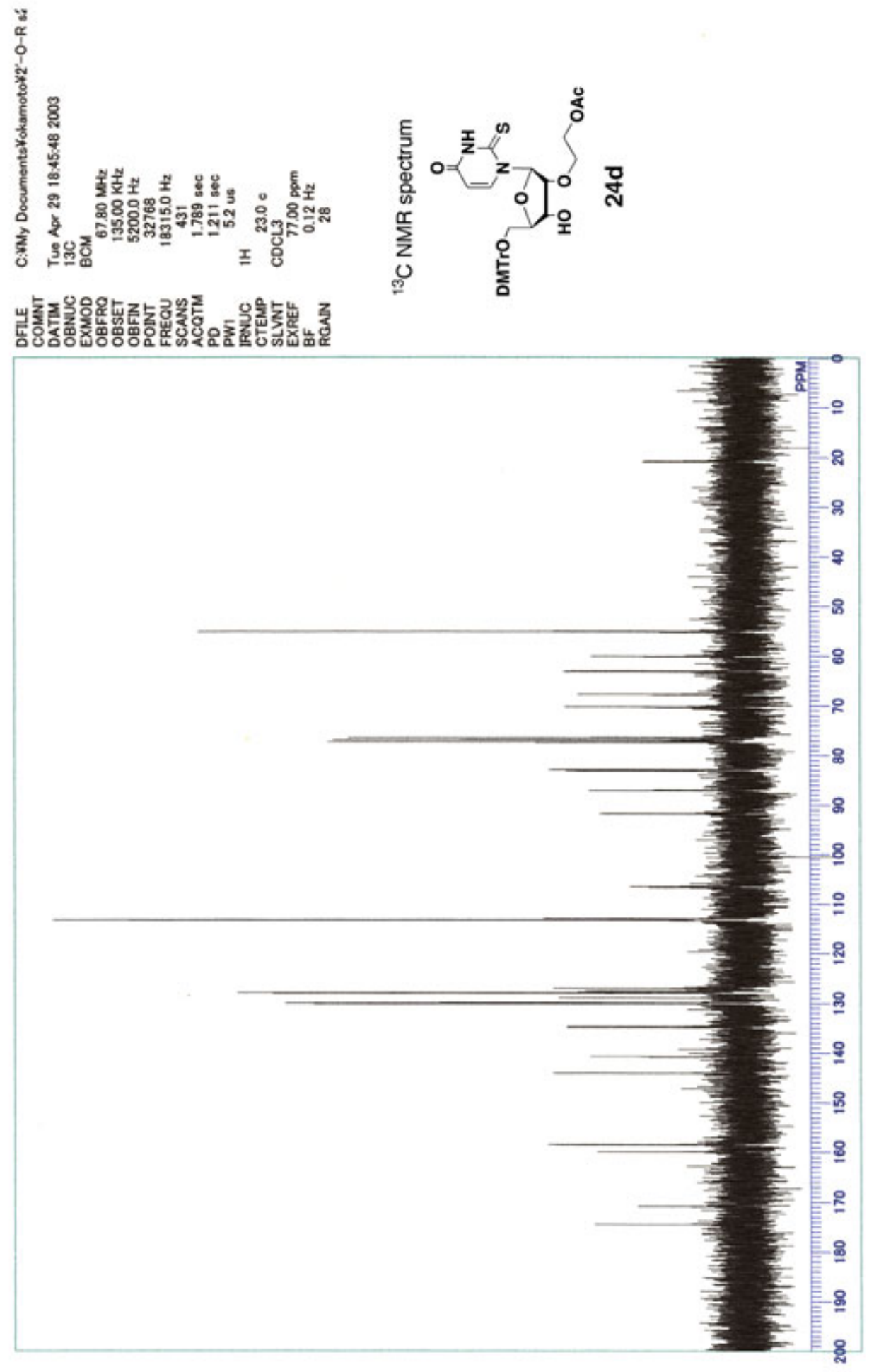




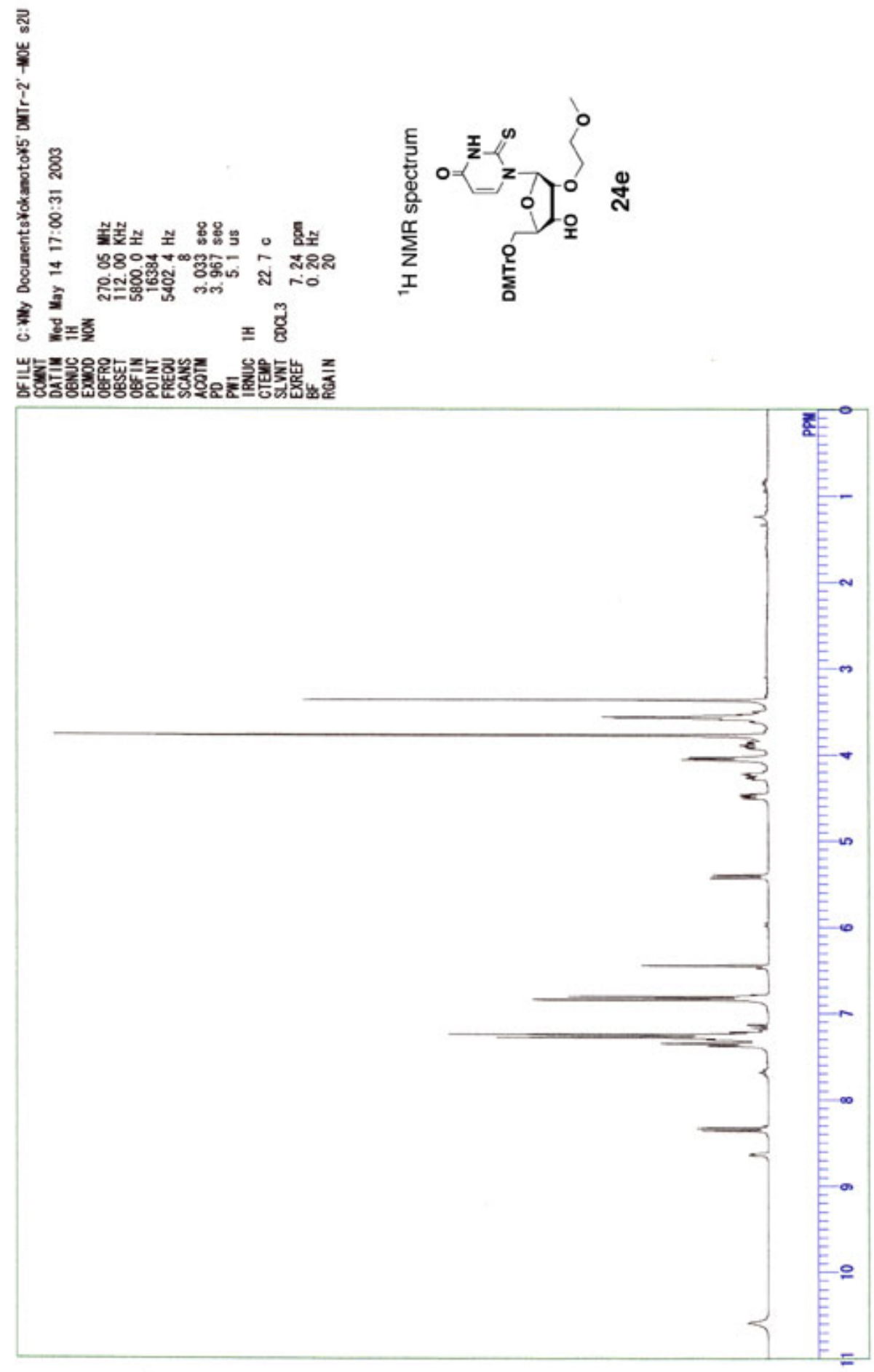



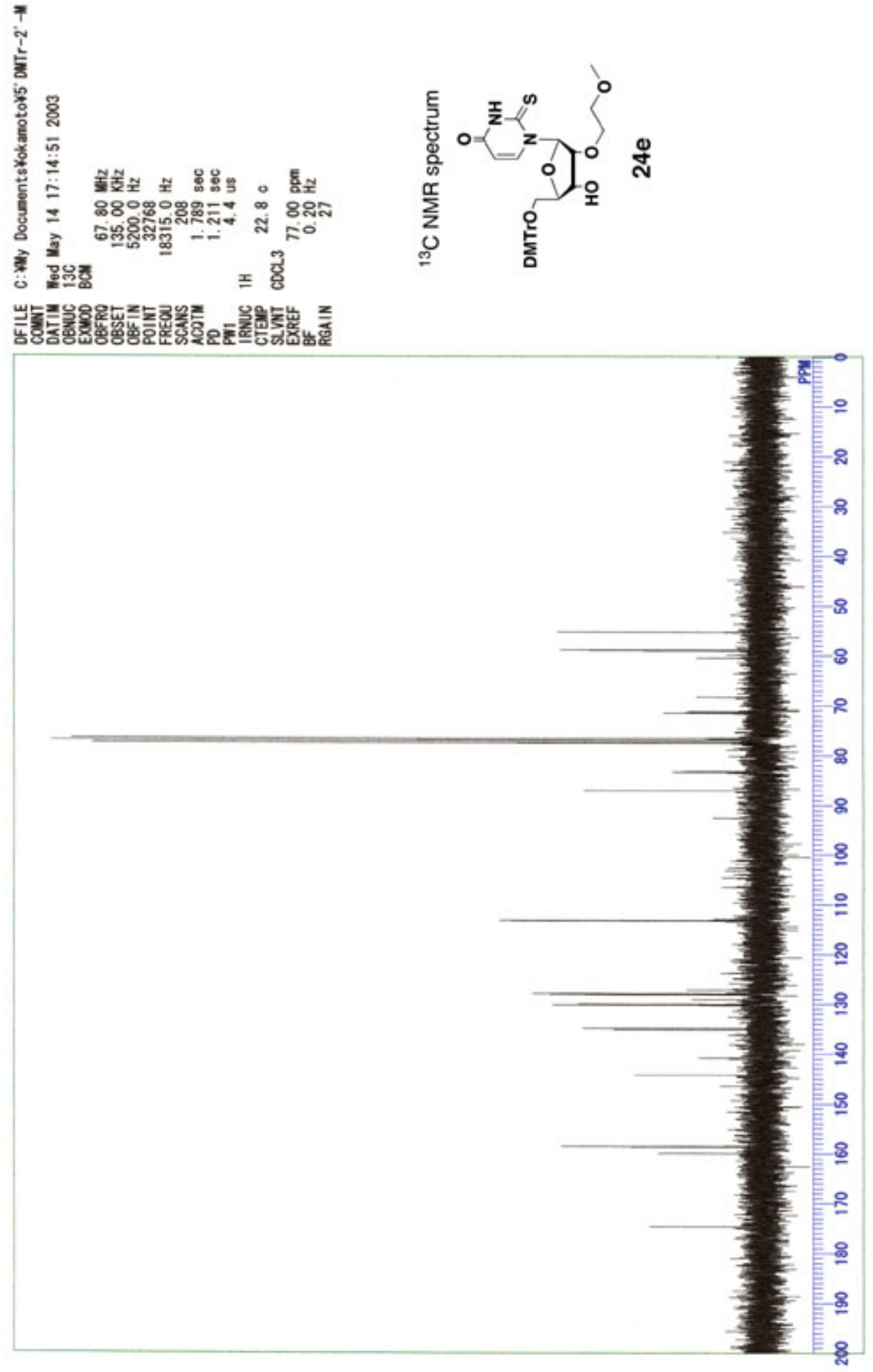


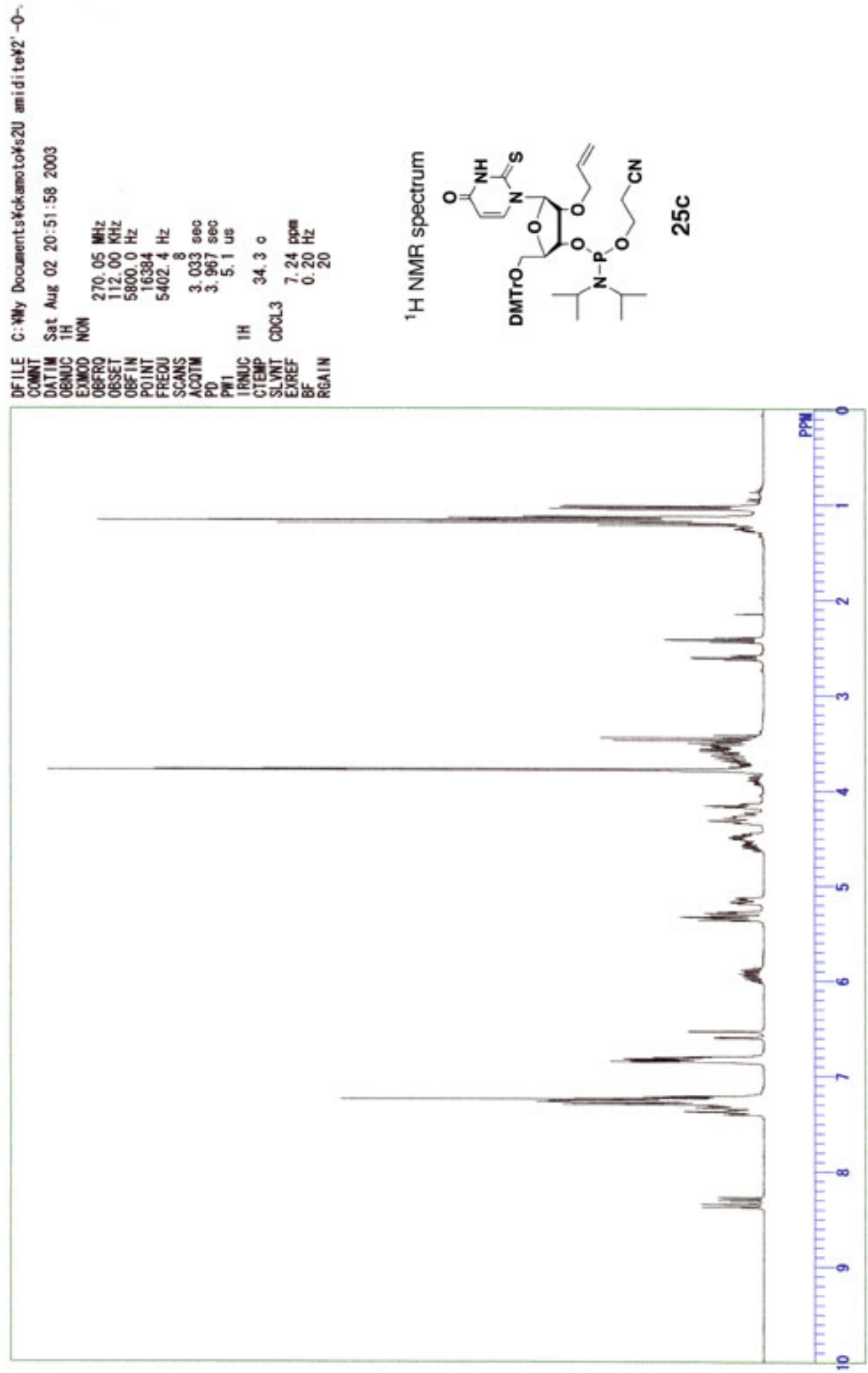



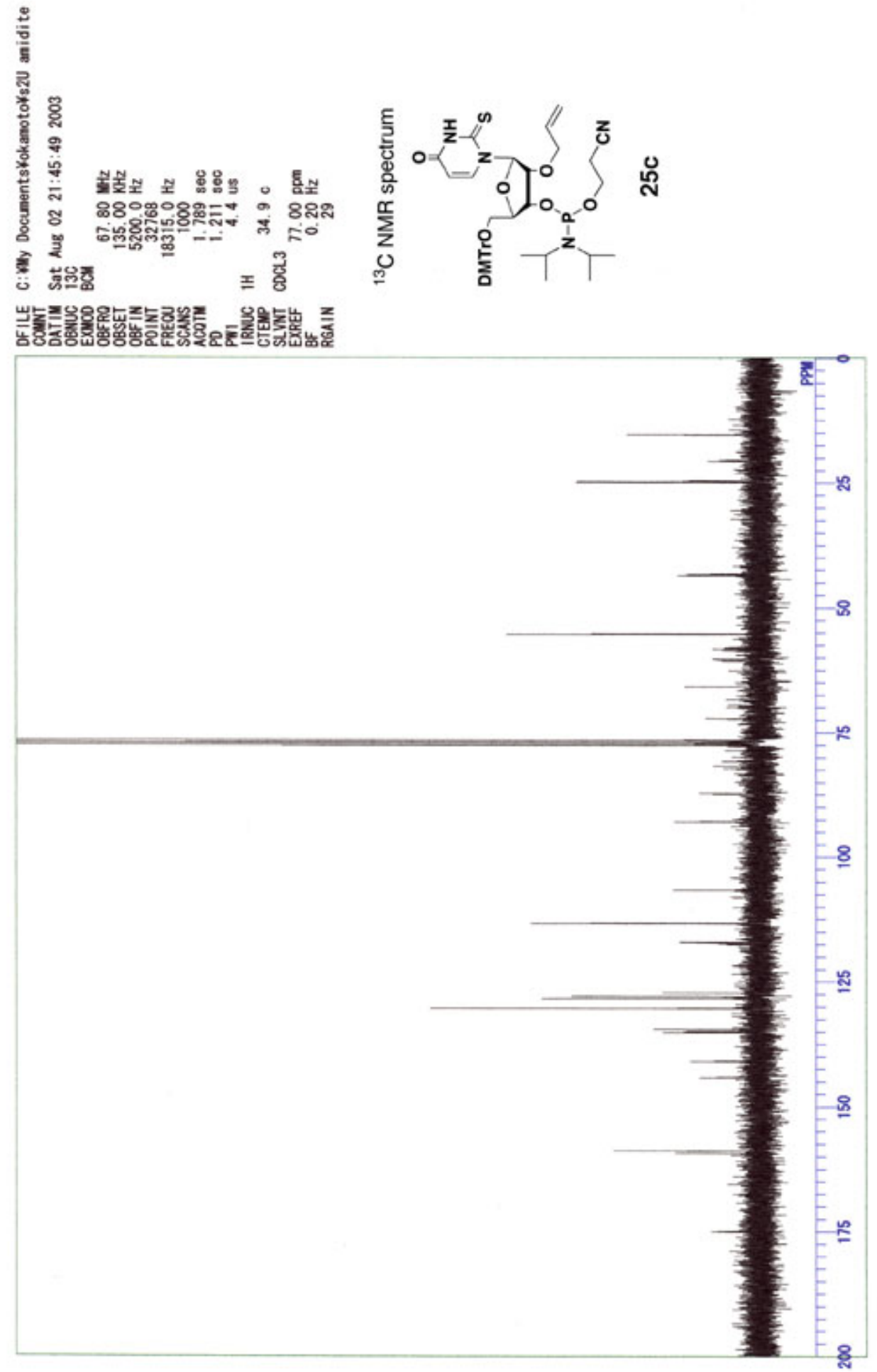

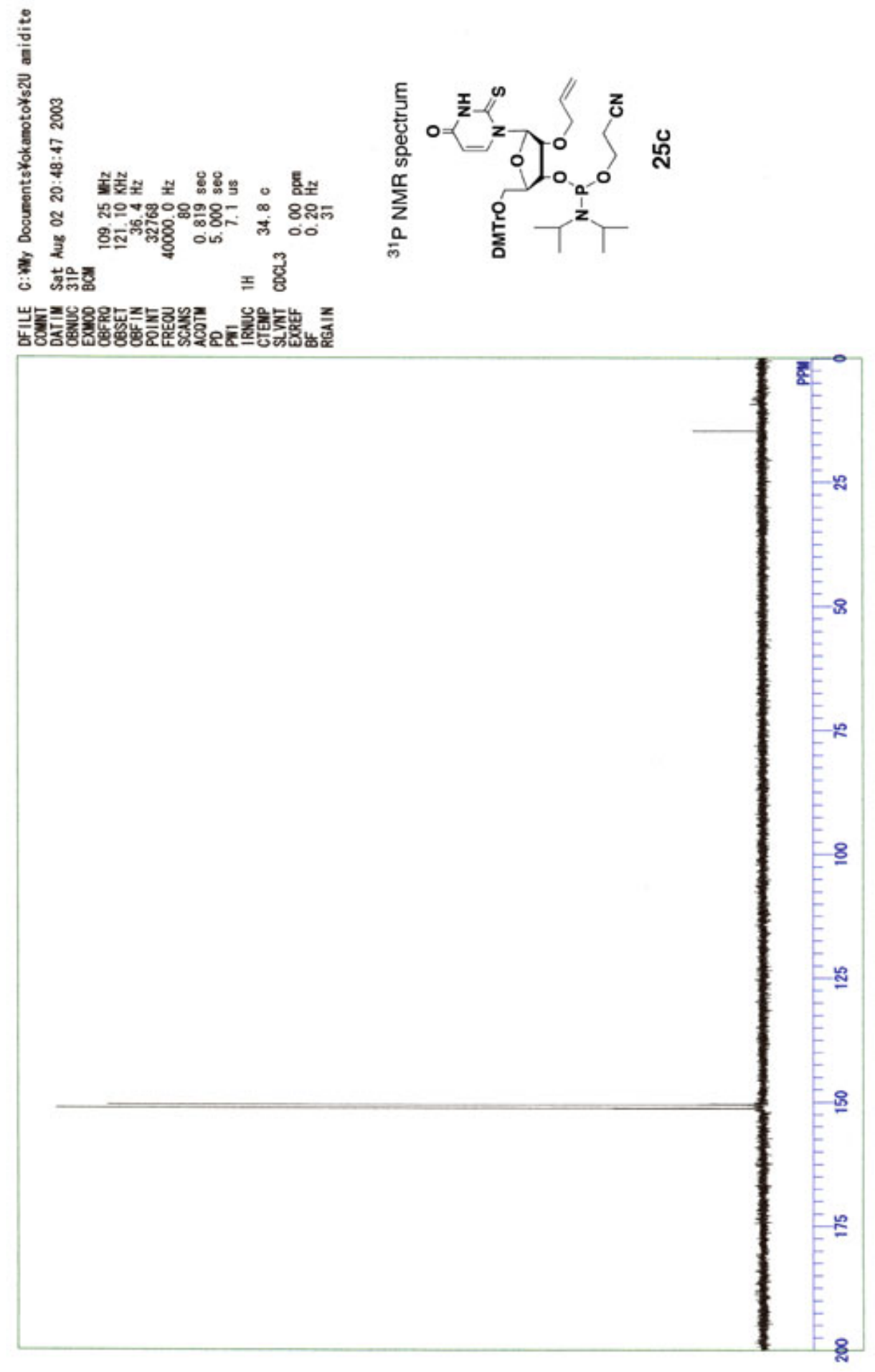


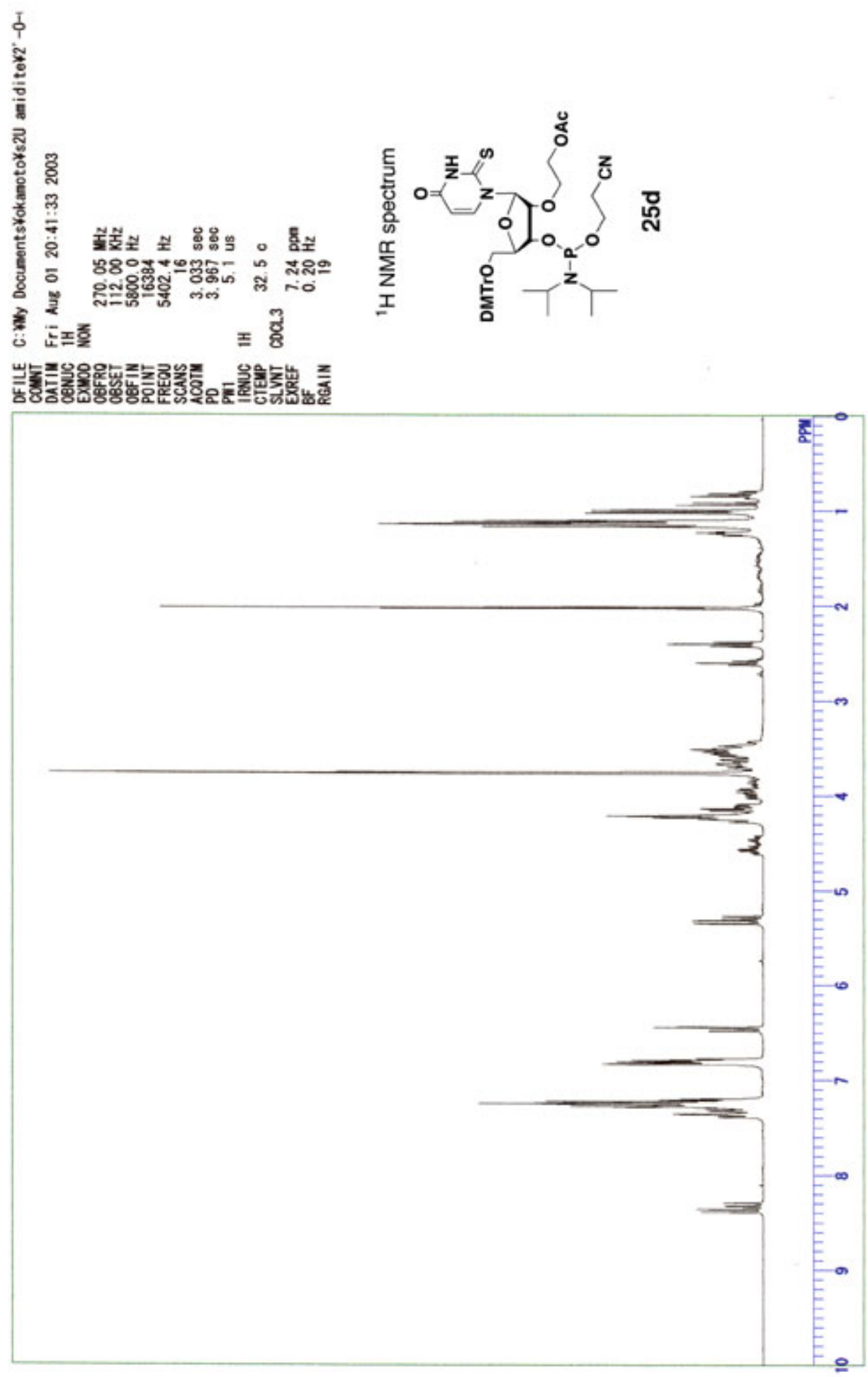




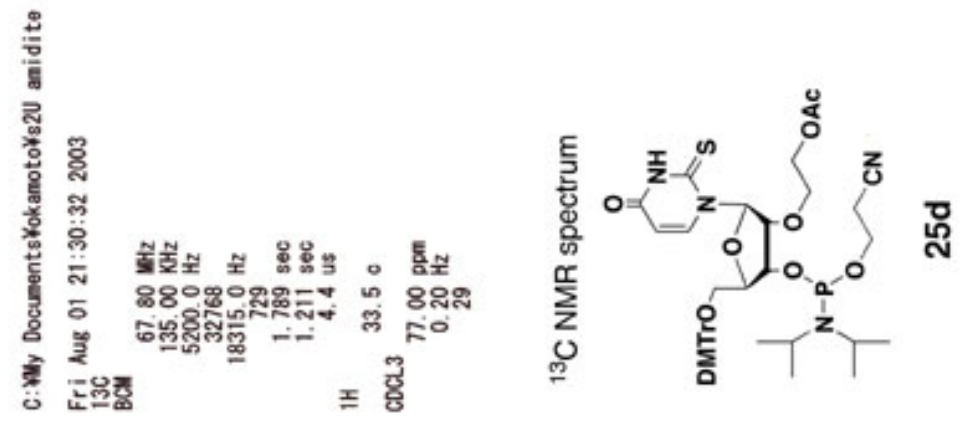

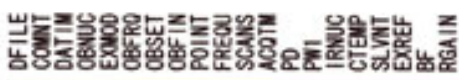

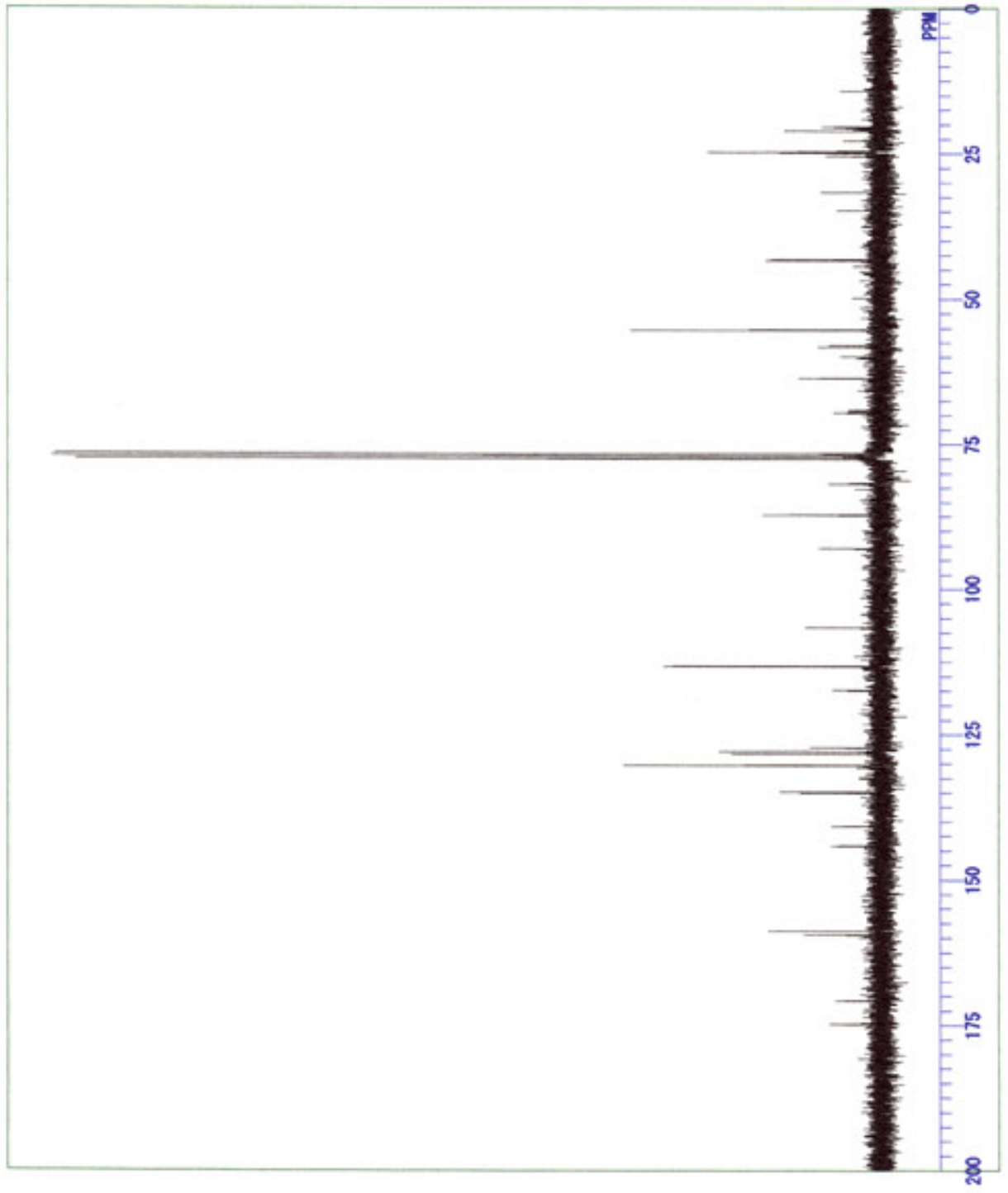




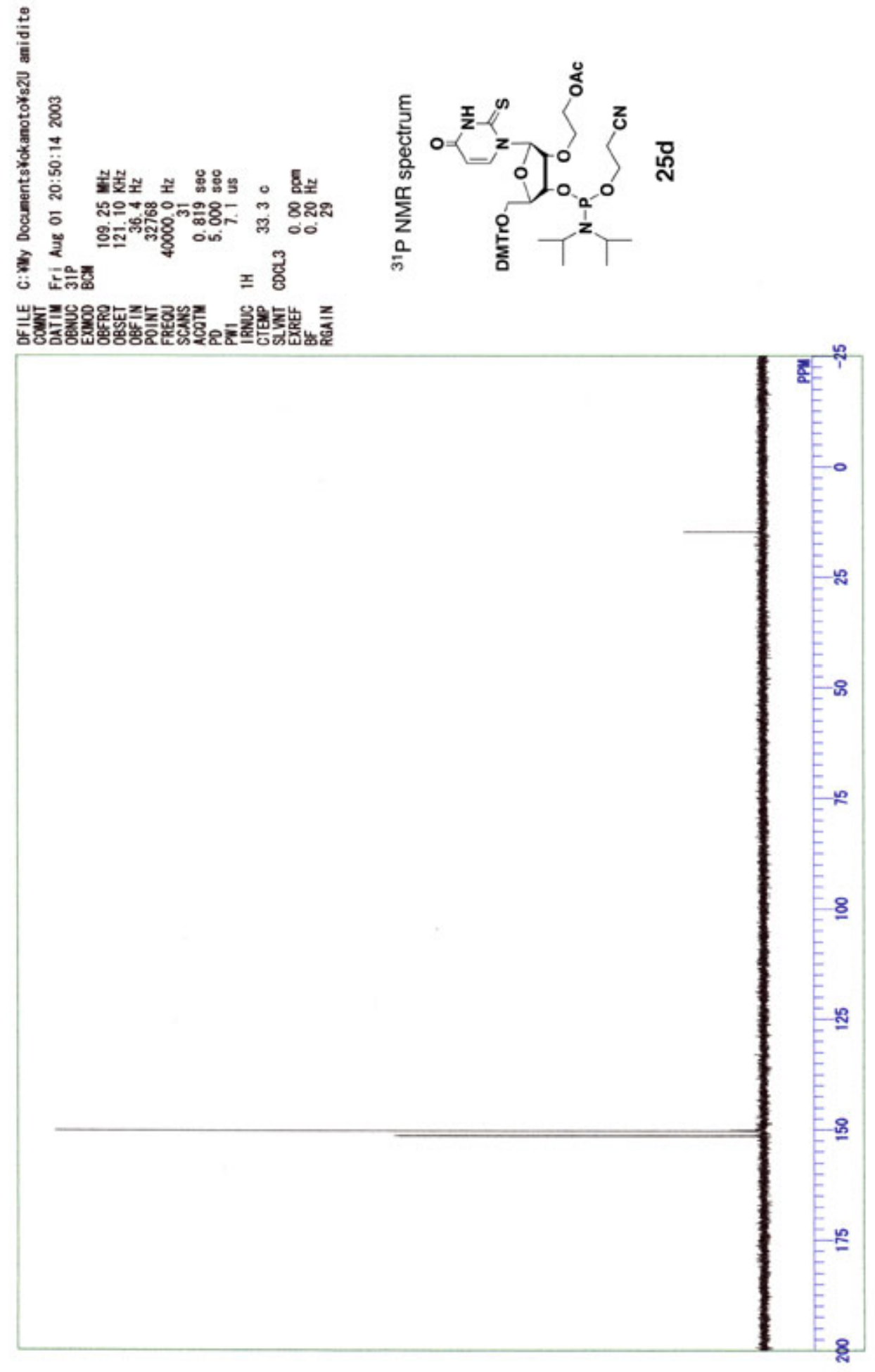

I 


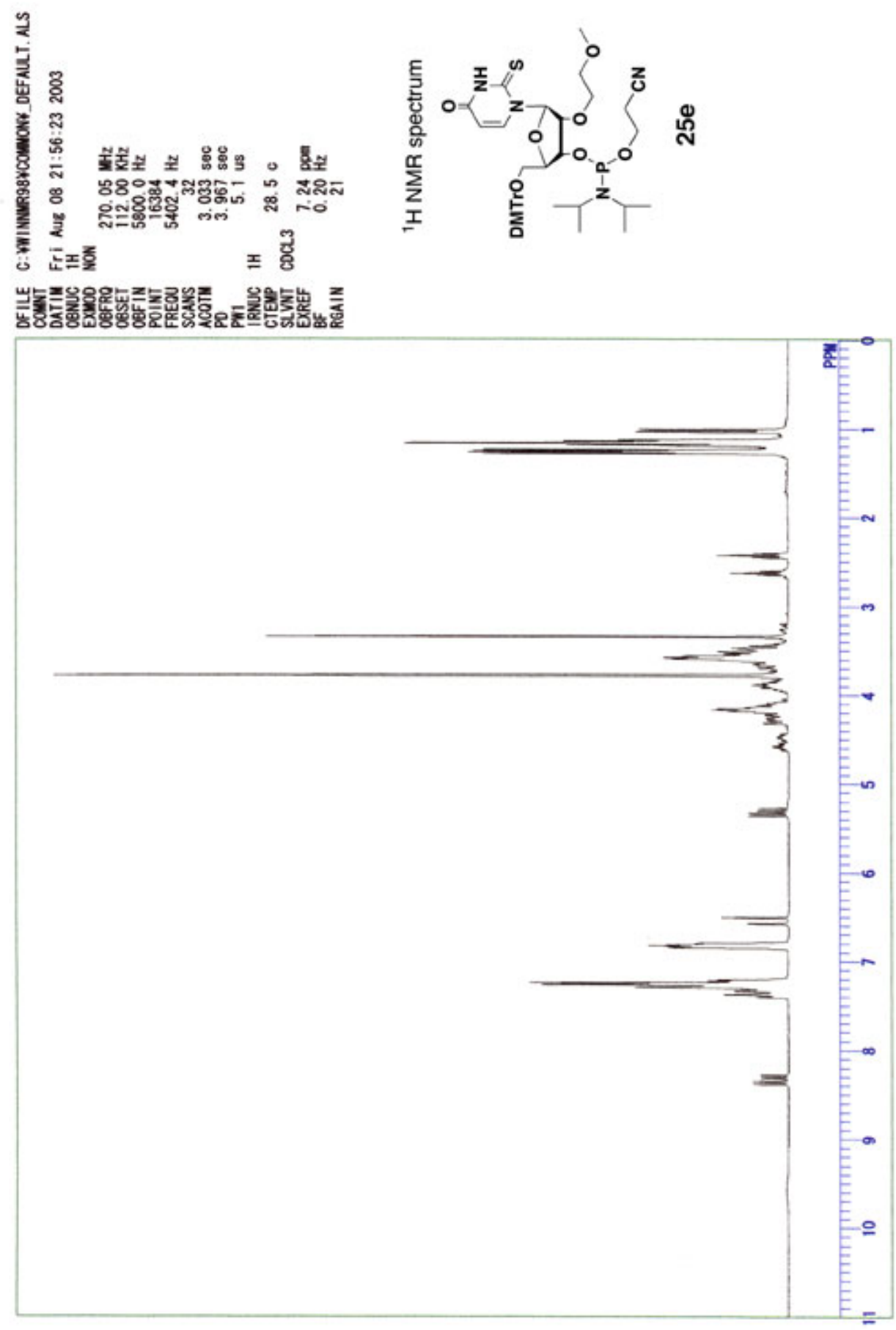




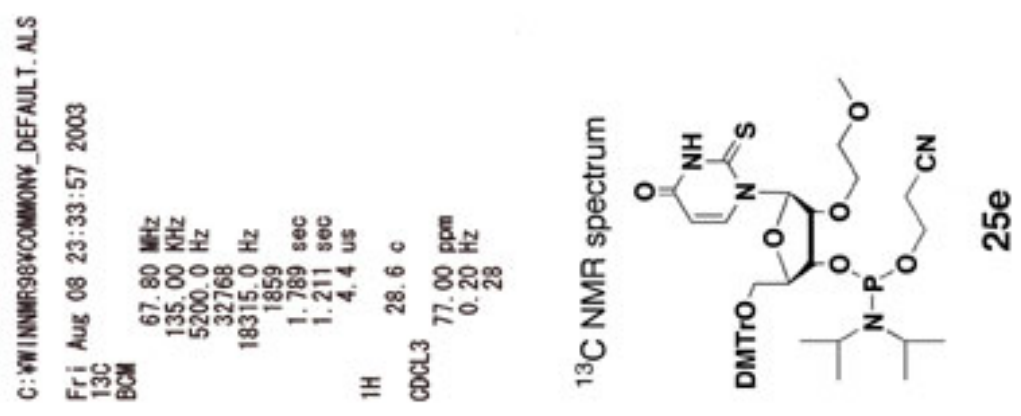

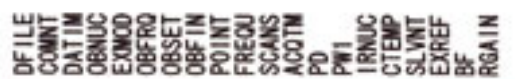

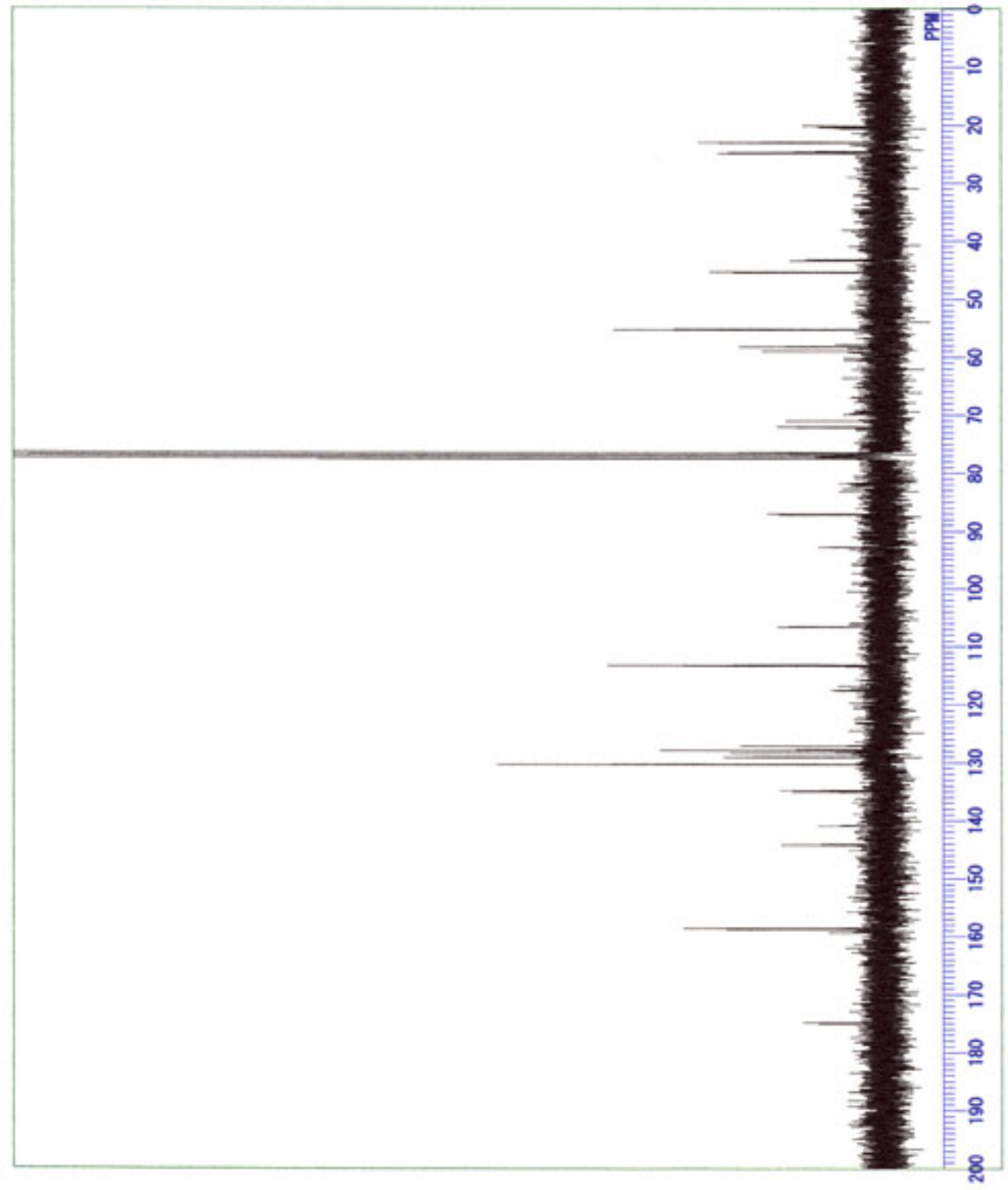




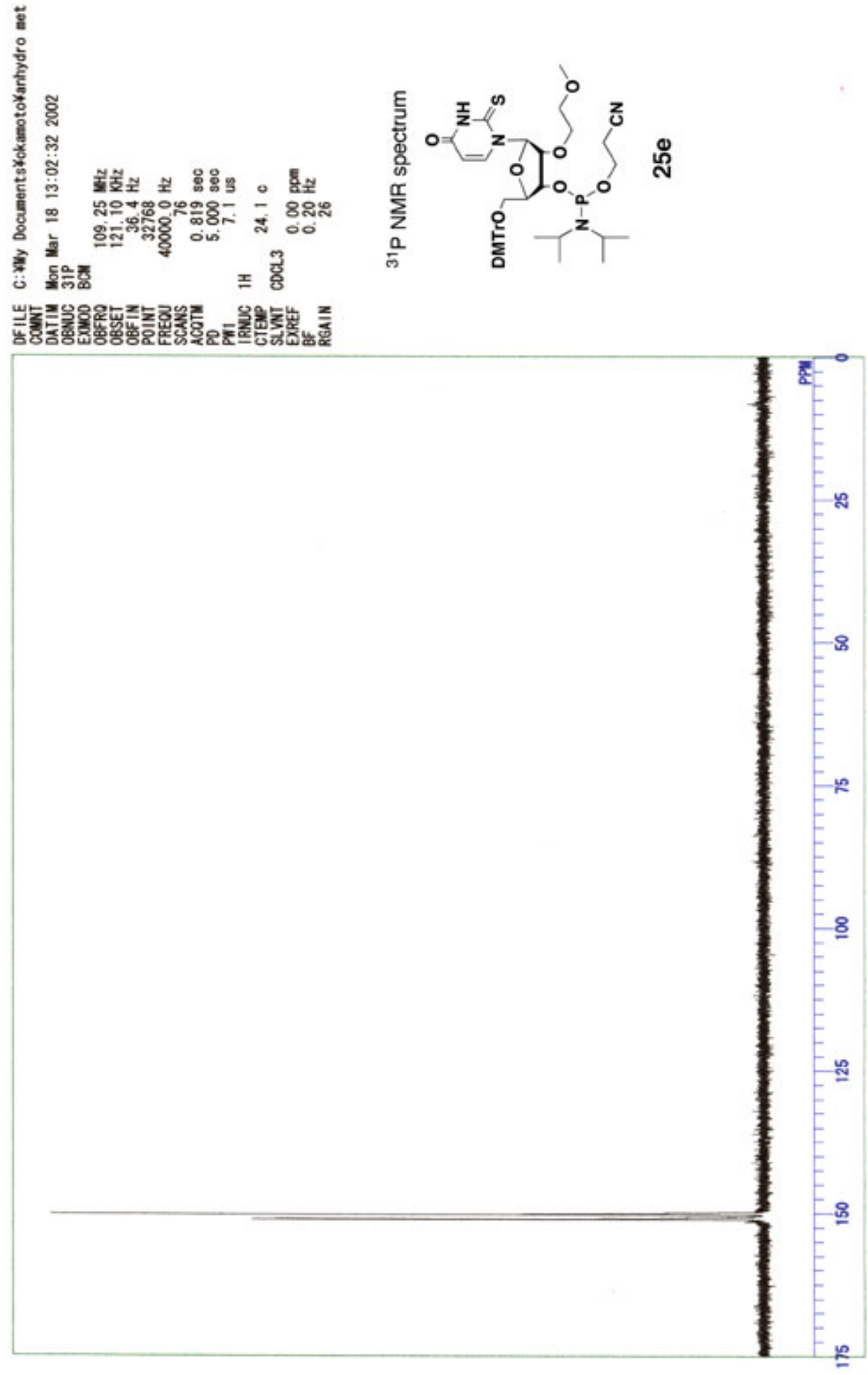

